Check for updates

Cite this: RSC Adv., 2018, 8, 18938

\title{
Synthesis of diversely substituted bis-pyrrolizidino/ thiopyrrolizidino oxindolo/acenaphthyleno curcuminoids via sequential azomethine ylide cycloaddition $\dagger$
}

\author{
Meenakshi Singh, a Abhijit Hazra, (D) *a Yogesh P. Bharitkar, (D) *a Ritu Kalia, ${ }^{a}$ \\ Ashutosh Sahoo, ${ }^{\mathrm{b}}$ Sneha Saha, ${ }^{a}$ V. Ravichandiran, ${ }^{a}$ Shekhar Ghosh ${ }^{\mathrm{b}}$ \\ and Nirup B. Mondal ${ }^{\mathrm{b}}$
}

Received 29th March 2018

Accepted 16th May 2018

DOI: $10.1039 / \mathrm{c} 8 \mathrm{ra02725k}$

rsc.li/rsc-advances

\begin{abstract}
Curcumin has been transformed to several diversely substituted bis-pyrrolizidino/thiopyrrolizidino oxindolo/acenaphthyleno curcuminoids via a sequential azomethine ylide cycloaddition reaction using isatins/acenaphthoquinone and proline/thioproline as the reagents. The products were separated via extensive chromatography and characterized by 1D/2D NMR and HRMS analysis.
\end{abstract}

\section{Introduction}

Incorporating diversity in the synthesis of combinatorial libraries of small molecules for biological screening is an emerging field. ${ }^{1}$ Rather than being directed toward a single biological target, diversified libraries can be used to identify new ligands for a variety of targets. It is hoped that the range of molecular architectures and potential bonding interactions present in a diversified library can provide interesting and specific biological activity across a range of targets. Although various chemical libraries are now available commercially, these remain focused primarily on so called 'drug-like' compounds. $^{2}$ Because these libraries are concentrated in a relatively narrow region of chemical structure space, it seems unlikely that they will provide useful probes for all biological targets of interest. ${ }^{3}$ The crucial factor for achieving success in drug discovery is not the size of the library but its structural diversity. ${ }^{4}$ Several different strategies for library design have therefore been developed to target the biologically relevant regions of chemical structure space. DOS has provided powerful probes to investigate biological mechanisms and also served as a new driving force for advancing synthetic organic chemistry.

To provide cyclic and heterocyclic compounds with a high degree of structural complexity as well as skeletal and

\footnotetext{
${ }^{a}$ National Institute of Pharmaceutical Education and Research (NIPER), (IICB Campus), 4, Raja S. C. Mullick Road, Jadavpur, Kolkata - 700 032, India

${ }^{b}$ Department of Organic and Medicinal Chemistry, Indian Institute of Chemical Biology, Council of Scientific and Industrial Research, 4, Raja S. C. Mullick Road, Jadavpur, Kolkata - 700 032, India. E-mail: apuhazra@gmail.com; yogeshbharitkar@gmail.com

$\dagger$ Electronic supplementary information (ESI) available: ${ }^{1} \mathrm{H},{ }^{13} \mathrm{C}$ NMR, HRMS data, spectral copies of all compounds associated with this article can be found. See DOI: $10.1039 / \mathrm{c} 8 \mathrm{ra} 02725 \mathrm{k}$
}

stereochemical diversity, dipolar cycloaddition reaction has emerged as a potential tool. ${ }^{5}$ Its ability to generate new stereocenters has allowed it to contribute very much to the development of stereo structure-activity relationships during screening campaigns. In particular, the sequential multicomponent reaction $^{6}$ and sequential azomethine ylide (1,3 dipole) cycloaddition approach has emerged as one of the efficient strategies which can provide diverse spirooxindoles in an operationally simple procedure from readily available chemical reagents. ${ }^{7}$ Keeping the above facts in mind we started our journey of preparing diversely functionalized heterocycles via azomethine ylide cycloaddition using simple commercially available ${ }^{8}$ or synthetic dipolarophiles. ${ }^{9}$ We then extended it to a new dimension by employing dipolarophiles available from nature like andrographolide, ${ }^{10}$ withaferin $A,{ }^{11}$ curcumin $^{12}$ etc. Several compounds have been prepared and biological activity evaluation revealed some very promising increment in activity. ${ }^{13,14}$ In continuation of our molecular diversity programme, very recently we have synthesized various spirooxindolo super curcumin analogues $^{12}$ to overcome the drawbacks related to the bioavailability of curcumin (less water solubility, easy metabolism and excretion) with comparable or better efficacy. An equally compelling motivation for their synthesis lies in their unique and formidable structure, the central feature being the biologically important curcumin and isatin (oxindole) units ${ }^{12}$ likewise done by various other group by synthsizing various diversified heterocyclic analogs of curcumin and their bioevaluation. ${ }^{15-18}$ We have so far succeeded in synthesizing a library of pyrrolizidino spirooxindolo curcumins, some with better and equal cytotoxic/antioxidant and antibacterial activity $^{19}$ but with much more specificity and solubility compared to curcumin. These results of biological evaluation encouraged us to construct a better diversified library applying 
the sequential azomethine ylide cycloaddition strategy, coupling isatin, substituted isatins or acenapthoquinone with proline or thioproline as the amino acid component.

\section{Results and discussion}

The first step in the two step sequential cycloaddition reaction was performed using curcumin (1), isatins (2A) and proline in $1: 1: 1$ mole ratio in refluxing methanol via in situ generation of azomethine ylides. In approximately $7-8 \mathrm{~h}$ the reactions delivered the mono cycloaddition products $( \pm)-3 \mathrm{Aa}(85 \%)$ and $( \pm)-\mathbf{3 A b}(5 \%)$ (Scheme 1). Major products $( \pm)-3 \mathbf{A a}$ were isolated and utilised in the second cycloaddition reaction with a differently substituted isatin and proline in equimolar ratio to produce four mixed pyrrolizidino spiro-oxindolo curcuminoids 4Aa-Ad with $80-85 \%$ total yield (Table 1).

The products (4Aa-Ad) were characterized from detailed spectral studies. All gave the same pseudo molecular ion peaks at $m / z 895[\mathrm{M}+\mathrm{H}]+$ and $917[\mathrm{M}+\mathrm{Na}]+$ in ESI-Q-TOF MS, indicating them to be isomeric. In the ${ }^{13} \mathrm{C}$ NMR spectra, they displayed 45 carbon signals due to the absence of symmetry (present in the previously reported ${ }^{12}$ diastereomers) due to the difference in substitution pattern in the two oxindole rings (originating from isatin substitution).

However, as in the previous publication, ${ }^{12}$ the signals for the aromatic ring of curcumin remained virtually unaltered in the spectra of the products. The chemical shifts for the nuclei belonging to the $\alpha, \beta$-unsaturated-diketone part of curcumin were of course distinctly perturbed, with $\mathrm{C} 3 / 25$ and $\mathrm{C} 4 / 26$ suffering profound alteration from downfield to upfield resonance positions.

It is obvious from Table 1 that in the entries $17-20$ and $21-24$ only the sequence of addition of 5-fluoroisatin (2E) and 5,7dimethylisatin (2F) has changed. This leads to the formation of $( \pm)$-4Ea-Ed and $( \pm)-\mathbf{4 F a}-\mathbf{F d}$. From a simple analysis of the

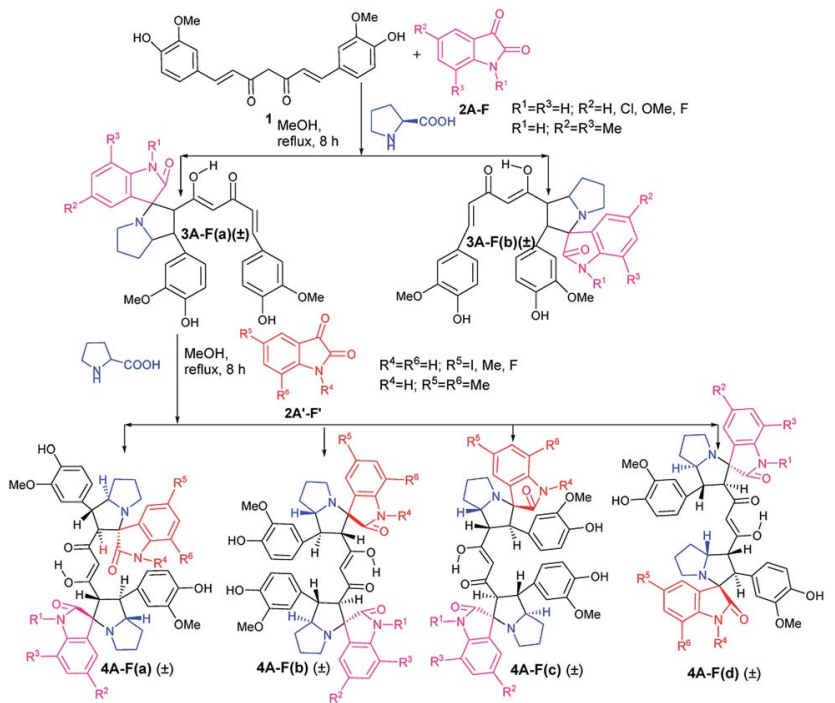

Scheme 1 Synthesis of mixed bis-pyrrolizidino dispiro-oxindolo curcuminoids.
Table 1 Yields of mixed bis-pyrrolizidino dispiro-oxindole 4(A-F)(a-d) derived from curcumin, isatins and proline

\begin{tabular}{lllllllll}
\hline Entry $^{a}$ & $\mathrm{R}^{1}$ & $\mathrm{R}^{2}$ & $\mathrm{R}^{3}$ & $\mathrm{R}^{4}$ & $\mathrm{R}^{5}$ & $\mathrm{R}^{6}$ & Product & Yield $^{b}(\%)$ \\
\hline 1 & $\mathrm{H}$ & $\mathrm{H}$ & $\mathrm{H}$ & $\mathrm{H}$ & $\mathrm{I}$ & $\mathrm{H}$ & $\mathbf{4 A a}$ & 20 \\
2 & $\mathrm{H}$ & $\mathrm{H}$ & $\mathrm{H}$ & $\mathrm{H}$ & $\mathrm{I}$ & $\mathrm{H}$ & $\mathbf{4 A b}$ & 23 \\
3 & $\mathrm{H}$ & $\mathrm{H}$ & $\mathrm{H}$ & $\mathrm{H}$ & $\mathrm{I}$ & $\mathrm{H}$ & $\mathbf{4 A c}$ & 21 \\
4 & $\mathrm{H}$ & $\mathrm{H}$ & $\mathrm{H}$ & $\mathrm{H}$ & $\mathrm{I}$ & $\mathrm{H}$ & $\mathbf{4 A d}$ & 24 \\
5 & $\mathrm{H}$ & $\mathrm{H}$ & $\mathrm{H}$ & $\mathrm{H}$ & $\mathrm{Me}$ & $\mathrm{Me}$ & $\mathbf{4 B a}$ & 19 \\
6 & $\mathrm{H}$ & $\mathrm{H}$ & $\mathrm{H}$ & $\mathrm{H}$ & $\mathrm{Me}$ & $\mathrm{Me}$ & $\mathbf{4 B b}$ & 22 \\
7 & $\mathrm{H}$ & $\mathrm{H}$ & $\mathrm{H}$ & $\mathrm{H}$ & $\mathrm{Me}$ & $\mathrm{Me}$ & $\mathbf{4 B c}$ & 21 \\
8 & $\mathrm{H}$ & $\mathrm{H}$ & $\mathrm{H}$ & $\mathrm{H}$ & $\mathrm{Me}$ & $\mathrm{Me}$ & $\mathbf{4 B d}$ & 23 \\
9 & $\mathrm{H}$ & $\mathrm{Cl}$ & $\mathrm{H}$ & $\mathrm{H}$ & $\mathrm{Me}$ & $\mathrm{H}$ & $\mathbf{4 C a}$ & 17 \\
10 & $\mathrm{H}$ & $\mathrm{Cl}$ & $\mathrm{H}$ & $\mathrm{H}$ & $\mathrm{Me}$ & $\mathrm{H}$ & $\mathbf{4 C b}$ & 20 \\
11 & $\mathrm{H}$ & $\mathrm{Cl}$ & $\mathrm{H}$ & $\mathrm{H}$ & $\mathrm{Me}$ & $\mathrm{H}$ & $\mathbf{4 C c}$ & 20 \\
12 & $\mathrm{H}$ & $\mathrm{Cl}$ & $\mathrm{H}$ & $\mathrm{H}$ & $\mathrm{Me}$ & $\mathrm{H}$ & $\mathbf{4 C d}$ & 23 \\
13 & $\mathrm{H}$ & $\mathrm{OMe}$ & $\mathrm{H}$ & $\mathrm{H}$ & $\mathrm{F}$ & $\mathrm{H}$ & $\mathbf{4 D a}$ & 18 \\
14 & $\mathrm{H}$ & $\mathrm{OMe}$ & $\mathrm{H}$ & $\mathrm{H}$ & $\mathrm{F}$ & $\mathrm{H}$ & $\mathbf{4 D b}$ & 22 \\
15 & $\mathrm{H}$ & $\mathrm{OMe}$ & $\mathrm{H}$ & $\mathrm{H}$ & $\mathrm{F}$ & $\mathrm{H}$ & $\mathbf{4 D c}$ & 21 \\
16 & $\mathrm{H}$ & $\mathrm{OMe}$ & $\mathrm{H}$ & $\mathrm{H}$ & $\mathrm{F}$ & $\mathrm{H}$ & $\mathbf{4 D d}$ & 24 \\
17 & $\mathrm{H}$ & $\mathrm{F}$ & $\mathrm{H}$ & $\mathrm{H}$ & $\mathrm{Me}$ & $\mathrm{Me}$ & $\mathbf{4 E a}$ & 18 \\
18 & $\mathrm{H}$ & $\mathrm{F}$ & $\mathrm{H}$ & $\mathrm{H}$ & $\mathrm{Me}$ & $\mathrm{Me}$ & $\mathbf{4 E b}$ & 22 \\
19 & $\mathrm{H}$ & $\mathrm{F}$ & $\mathrm{H}$ & $\mathrm{H}$ & $\mathrm{Me}$ & $\mathrm{Me}$ & $\mathbf{4 E c}$ & 21 \\
20 & $\mathrm{H}$ & $\mathrm{F}$ & $\mathrm{H}$ & $\mathrm{H}$ & $\mathrm{Me}$ & $\mathrm{Me}$ & $\mathbf{4 E d}$ & 23 \\
21 & $\mathrm{H}$ & $\mathrm{Me}$ & $\mathrm{Me}$ & $\mathrm{H}$ & $\mathrm{F}$ & $\mathrm{H}$ & $\mathbf{4 F a}$ & 17 \\
22 & $\mathrm{H}$ & $\mathrm{Me}$ & $\mathrm{Me}$ & $\mathrm{H}$ & $\mathrm{F}$ & $\mathrm{H}$ & $\mathbf{4 F b}$ & 20 \\
23 & $\mathrm{H}$ & $\mathrm{Me}$ & $\mathrm{Me}$ & $\mathrm{H}$ & $\mathrm{F}$ & $\mathrm{H}$ & $\mathbf{4 F c}$ & 20 \\
24 & $\mathrm{H}$ & $\mathrm{Me}$ & $\mathrm{Me}$ & $\mathrm{H}$ & $\mathrm{F}$ & $\mathrm{H}$ & $\mathbf{4 F d}$ & 22 \\
& & & & & & & &
\end{tabular}

${ }^{a}$ Unless otherwise noted, the reaction was performed with $1.76 \mathrm{mmol}$ of 3A-F(a) $( \pm)$, isatins and proline in $50.0 \mathrm{~mL}$ of $\mathrm{MeOH}$ under reflux for $8 \mathrm{~h}$. ${ }^{b}$ Determined after isolation.

reaction profile and structure of the product it could be easily concluded that $\mathbf{4 E a}, \mathbf{4 E b}$ are identical with $4 \mathrm{Fa}, \mathbf{4 F b}$ respectively whereas $4 \mathbf{E c}$, $\mathbf{4 E d}$ are not identical with $\mathbf{4 F c}$ and $\mathbf{4 F d}$ due to different substitution present in oxindole ring. In fact this was also proved from the detailed NMR spectral comparison of all the products. Detailed possibilities in change of sequential addition with different isatins have been schematically

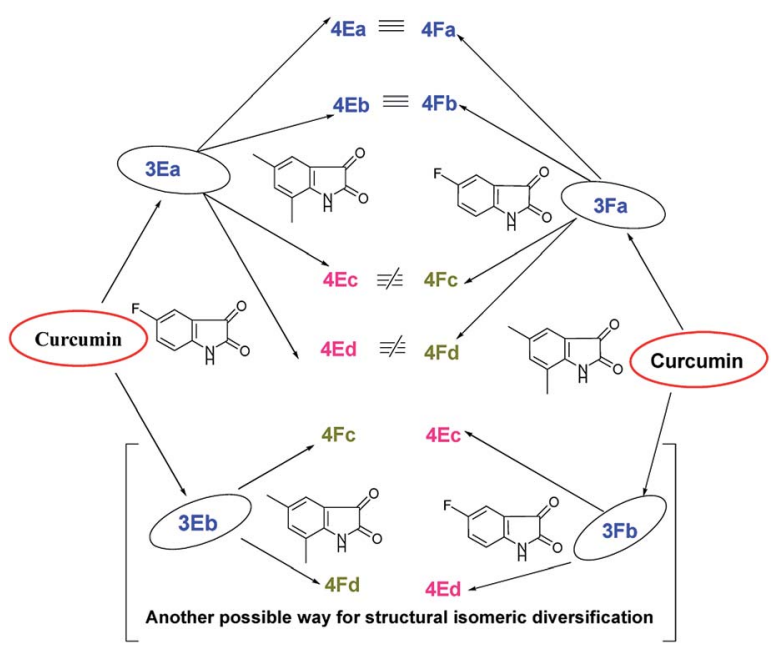

Fig. 1 Possible mixed bis-pyrrolizidino dispiro-oxindolo curcuminoids via change in sequential addition of any two substituted isatins. 
represented using 5-fluoroisatin (2E) and 5,7-dimethylisatin (2F) as model example in Fig. 1.

Because of the possibility of formation of two other diastereomers $( \pm)-\mathbf{4}(\mathbf{A}-\mathbf{F}) \mathbf{c}^{\prime}$ and $( \pm)-\mathbf{4}(\mathbf{A}-\mathbf{F}) \mathbf{d}^{\prime}$ (Fig. 1), positional isomers of $( \pm)-\mathbf{4}(\mathbf{A}-\mathbf{F}) \mathbf{c}$ and $( \pm)-\mathbf{4}(\mathbf{A}-\mathbf{F}) \mathbf{d}$ due to different substitution in isatin ring, there is the extra advantage of more diversification. But it is necessary to separate $( \pm)-\mathbf{3}(\mathbf{A}-\mathbf{F}) \mathbf{a}$ and $\mathbf{3}(\mathbf{A}-\mathbf{F}) \mathbf{b} /( \pm)-\mathbf{3}(\mathbf{A}-\mathbf{F})$ $\mathbf{a}^{\prime}$ and 3(A-F) $\mathbf{b}^{\prime}$ synthesized in the first step to avoid formation of complex mixture of compounds in one pot sequence reaction, difficult to separate.

Following the success in the synthesis of mixed bispyrrolizidino dispiro-oxindolo curcuminoids we wanted to synthesize mixed pyrrolizidino-thiopyrrolizidino dispirooxindolo curcuminoids (Scheme 2). But to confirm that the reaction indeed proceeds with thioproline (thiazolidine-4carboxylic acid), we treated curcumin and isatins with thiazolidine-4-carboxylic acid in $1: 2: 2$ mole ratio. In this case we obtained only two diastereomers $( \pm)-5 \mathbf{A a}$ and $( \pm)-\mathbf{5 A b}$ in around $70 \%$ yield in $15-16 \mathrm{~h}$; both are symmetric in nature (confirmed from the 21 carbon peaks present in ${ }^{13} \mathrm{C} N M R$ ).

Similarly, when we used mono cycloaddition product $( \pm)$-3Aa with a differently substituted isatin and thiazolidine-4carboxylic acid in 1:1:1 mole ratio, we could isolate only two diastereomers of mixed pyrrolizidino-thiopyrrolizidino dispiro-oxindolo curcuminoids after $8 \mathrm{~h}$ of reaction (Scheme 3, Table 2).

In case of the reactions where thioproline was used, the reaction rate and also the yield of the product were lower

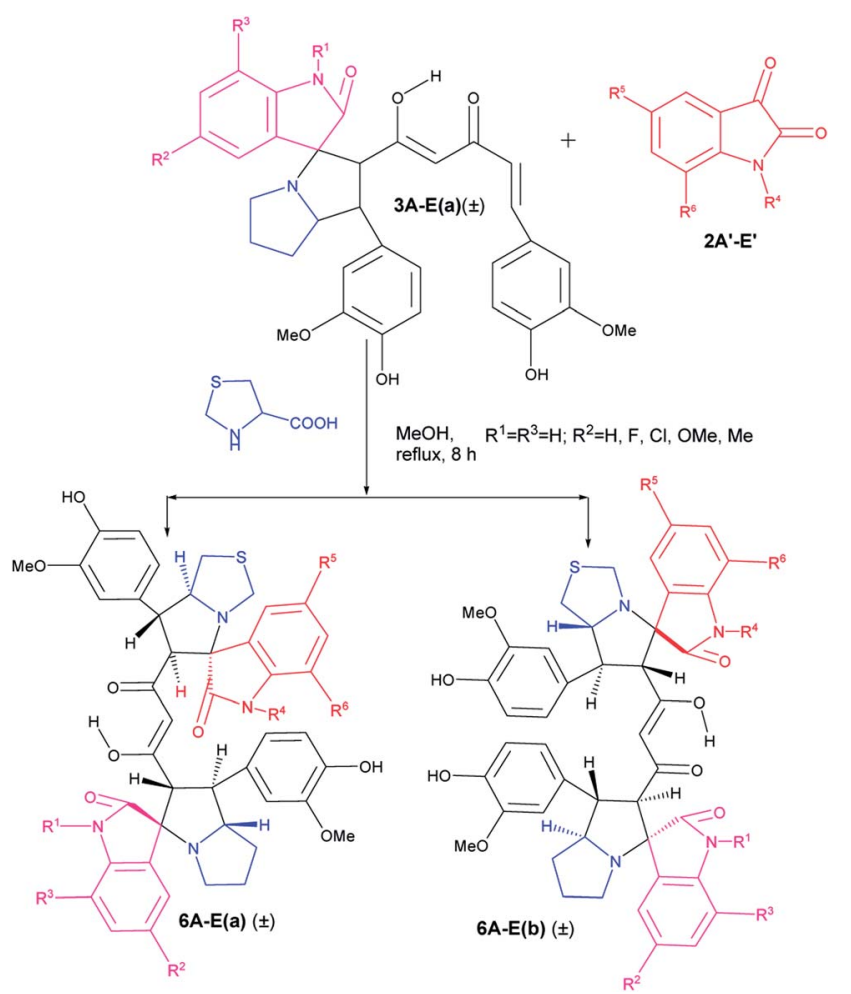

Scheme 2 Synthesis of bis-thiopyrrolizidino dispiro-oxindolo curcuminoids.

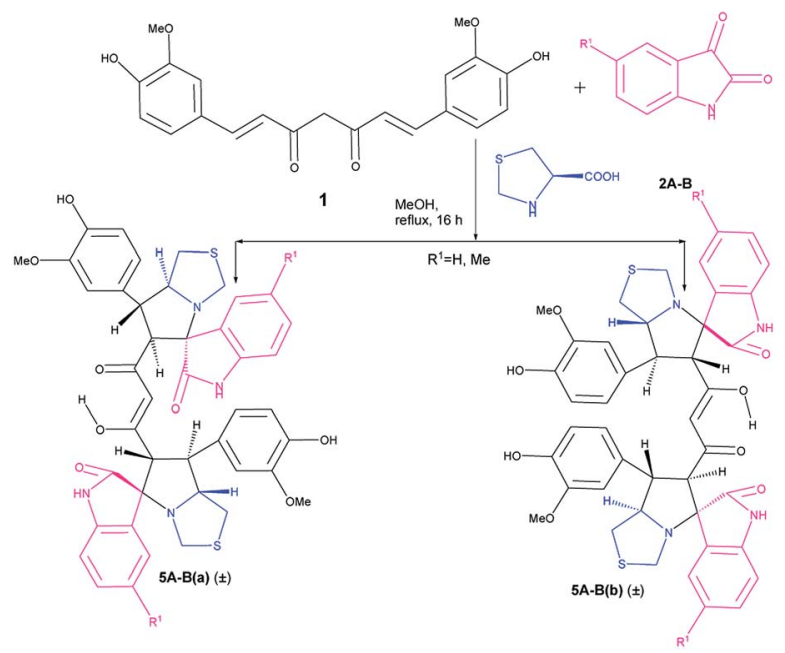

Scheme 3 Synthesis of mixed pyrrolizidino-thiopyrrolizidino dispirooxindolo curcuminoids.

compared to those with proline perhaps due to lower solubility of thiazolidine-4-carboxylic acid (Table 2) in methanol.

As usual the products were characterized from detailed spectral studies. Both the products $( \pm)-6 \mathbf{A a}$ and $( \pm)-6 \mathbf{A b}$ gave the same peaks at $m / z 817[\mathrm{M}+\mathrm{H}]^{+} / 839[\mathrm{M}+\mathrm{Na}]^{+}$in the ESI-QTOF MS spectrum for the pseudomolecular ions, indicating them to be isomeric. Both showed 45 carbon signals in ${ }^{13} \mathrm{C}$ NMR spectra indicating a break in symmetry due to the change in amino acid as well as for the difference in substitution in the isatin ring.

The crucial evidence in support of the proposed structures came from the observed HMBC correlation in the 2D NMR spectrum of $\mathbf{6 A a} / \mathbf{A b}$ between signals of $\mathbf{C}-10 / 32$ (i.e., the oxindole carbonyls, $\delta$ 181.0/179.9) and $\mathrm{H}-3 / 25$ ( $\delta$ 4.49/4.36) and signals of $\mathrm{C}-5 / 27$ (i.e., the point of attachment with proline, $\delta 73.9 / 76.5)$ and C-6/28 ( $\delta 30.9 / 36.7)$ with $\mathrm{H}-4 / 26(\delta 3.70 / 3.63)$. Further, the COSY relationship between $\mathrm{H}-4 / 26$ ( $\delta$ 3.70/3.63) and $\mathrm{H}-5 / 27$ ( $\delta 4.23 / 4.32$ ), coupled with medium to low NOESY cross peaks, strongly supports the mode of additions (Fig. 2).

Table 2 Yields of mixed bis-thiopyrrolizidino dispiro-oxindole 6(AE)(a-b) derived from curcumin, isatins, proline and thioproline

\begin{tabular}{lllllllll}
\hline Entry $^{a}$ & $\mathrm{R}^{1}$ & $\mathrm{R}^{2}$ & $\mathrm{R}^{3}$ & $\mathrm{R}^{4}$ & $\mathrm{R}^{5}$ & $\mathrm{R}^{6}$ & Product & Yield $^{b}(\%)$ \\
\hline 1 & $\mathrm{H}$ & $\mathrm{H}$ & $\mathrm{H}$ & $\mathrm{H}$ & $\mathrm{OMe}$ & $\mathrm{H}$ & $\mathbf{6 A a}$ & 30 \\
2 & $\mathrm{H}$ & $\mathrm{H}$ & $\mathrm{H}$ & $\mathrm{H}$ & $\mathrm{OMe}$ & $\mathrm{H}$ & $\mathbf{6 A b}$ & 35 \\
3 & $\mathrm{H}$ & $\mathrm{F}$ & $\mathrm{H}$ & $\mathrm{H}$ & $\mathrm{I}$ & $\mathrm{H}$ & $\mathbf{6 B a}$ & 32 \\
4 & $\mathrm{H}$ & $\mathrm{F}$ & $\mathrm{H}$ & $\mathrm{H}$ & $\mathrm{I}$ & $\mathrm{H}$ & $\mathbf{6 B b}$ & 37 \\
5 & $\mathrm{H}$ & $\mathrm{Cl}$ & $\mathrm{H}$ & $\mathrm{H}$ & $\mathrm{I}$ & $\mathrm{H}$ & $\mathbf{6 C a}$ & 31 \\
6 & $\mathrm{H}$ & $\mathrm{Cl}$ & $\mathrm{H}$ & $\mathrm{H}$ & $\mathrm{I}$ & $\mathrm{H}$ & $\mathbf{6 C b}$ & 35 \\
7 & $\mathrm{H}$ & $\mathrm{OMe}$ & $\mathrm{H}$ & $\mathrm{H}$ & $\mathrm{Me}$ & $\mathrm{Me}$ & $\mathbf{6 D a}$ & 32 \\
8 & $\mathrm{H}$ & $\mathrm{OMe}$ & $\mathrm{H}$ & $\mathrm{H}$ & $\mathrm{Me}$ & $\mathrm{Me}$ & $\mathbf{6 D b}$ & 36 \\
9 & $\mathrm{H}$ & $\mathrm{Me}$ & $\mathrm{H}$ & $\mathrm{H}$ & $\mathrm{Me}$ & $\mathrm{Me}$ & $\mathbf{6 E a}$ & 31 \\
10 & $\mathrm{H}$ & $\mathrm{Me}$ & $\mathrm{H}$ & $\mathrm{H}$ & $\mathrm{Me}$ & $\mathrm{Me}$ & $\mathbf{6 E b}$ & 36
\end{tabular}

${ }^{a}$ Unless otherwise noted, the reaction was performed with $1.76 \mathrm{mmol}$ of $3 \mathrm{~A}-\mathrm{E}(\mathrm{a})( \pm)$, isatins and thioproline in $50.0 \mathrm{~mL}$ of $\mathrm{MeOH}$ under reflux for 8 h. ${ }^{b}$ Determined after isolation. 


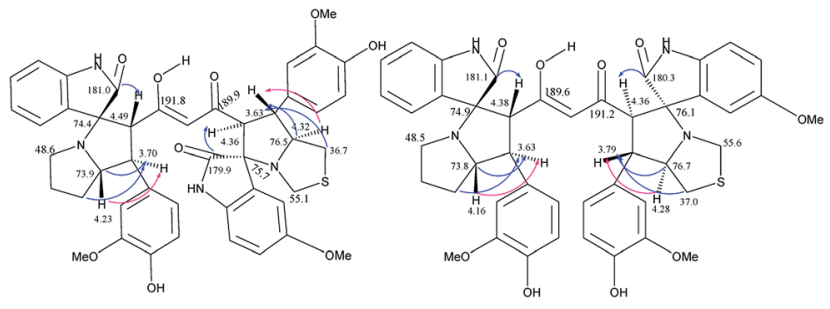

Fig. 2 Important correlations of $6 \mathrm{Aa}$ and $6 \mathrm{Ab}[\mathrm{HMBC}(\longrightarrow)$ $\cos (\rightarrow)]$.

It is worth mentioning here that the adjacent hydrogens on both side of the diketone functionality of the curcumin unit are anti to each other, whereas they were in syn orientation with the spirocyclic oxindole bond of the respective sides.

The success achieved in the synthesis of mixed pyrrolizidino-thiopyrrolizidino dispiro-oxindolo curcuminoids prompted us for the synthesis of a new type of diversely substituted product using isatin, acenaphthoquinone and proline/thiproline in various combinations. We used mono cycloaddition product $( \pm)-3 \mathbf{A a}$, acenaphthoquinone and proline/thioproline in $1: 1: 1$ molar ratio and isolated only one isomer of mixed bis-pyrrolizidino-dispirooxindolo-acenaphthyleno curcuminoids/bis-pyrrolizidinothiopyrrolizidino dispiro-oxindolo-acenaphthyleno curcuminoids (7A-D) after $8 \mathrm{~h}$ of reaction (Scheme 4) with moderate yield $(45 \%$, Table 3$)$ although the formation of minor stereoisomers could not be ruled out.

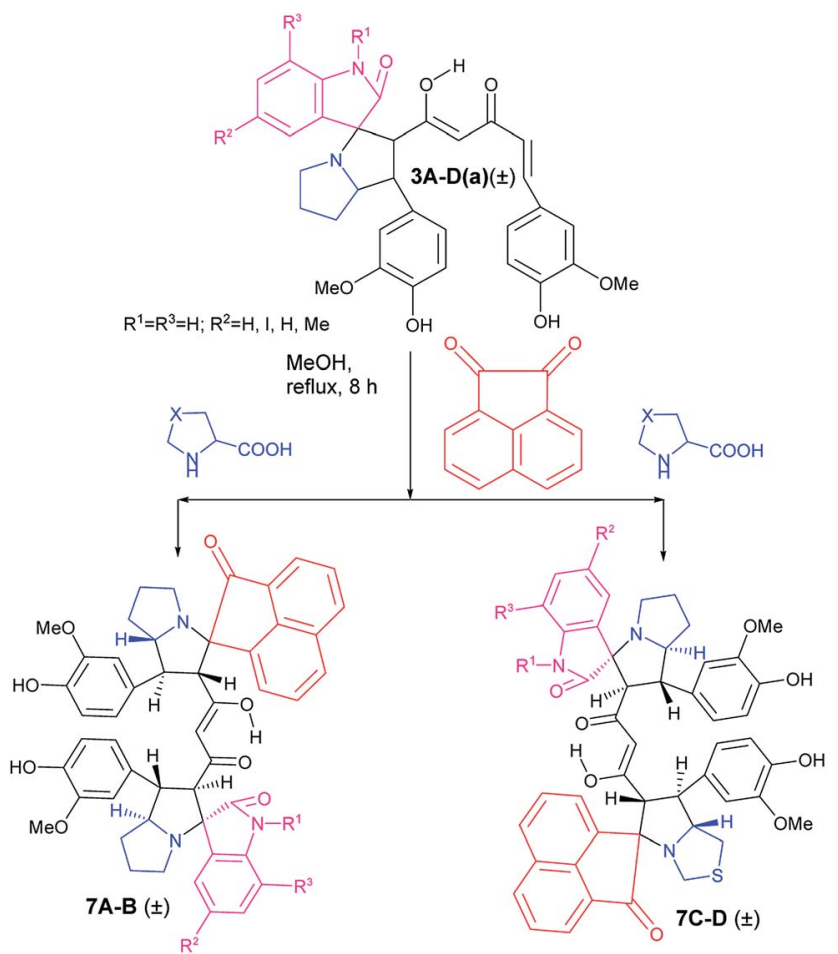

Scheme 4 Synthesis of mixed bis-pyrrolizidino/-thiopyrrolizidino dispiro-oxindolo-acenapthylino curcuminoids.
Table 3 Yields of mixed bis-pyrrolizidino/-thiopyrrolizidino dispirooxindolo-acenaphthylino curcuminoids 7A-D

\begin{tabular}{lllllll}
\hline Entry $^{a}$ & $\mathrm{R}^{1}$ & $\mathrm{R}^{2}$ & $\mathrm{R}^{3}$ & $\mathrm{X}$ & Product & Yield $^{b}(\%)$ \\
\hline 1 & $\mathrm{H}$ & $\mathrm{H}$ & $\mathrm{H}$ & $-\mathrm{CH}_{2}$ & $7 \mathbf{A}$ & 54 \\
2 & $\mathrm{H}$ & $\mathrm{I}$ & $\mathrm{H}$ & $-\mathrm{CH}_{2}$ & $7 \mathrm{~B}$ & 56 \\
3 & $\mathrm{H}$ & $\mathrm{H}$ & $\mathrm{H}$ & $-\mathrm{S}$ & $7 \mathrm{C}$ & 50 \\
4 & $\mathrm{H}$ & $\mathrm{Me}$ & $\mathrm{H}$ & $-\mathrm{S}$ & 7D & 49
\end{tabular}

${ }^{a}$ Unless otherwise noted, the reaction was performed with $1.76 \mathrm{mmol}$ of 3A-D(a) $( \pm)$, isatins and proline in $50.0 \mathrm{~mL}$ of $\mathrm{MeOH}$ under reflux for $8 \mathrm{~h}$. ${ }^{b}$ Determined after isolation.

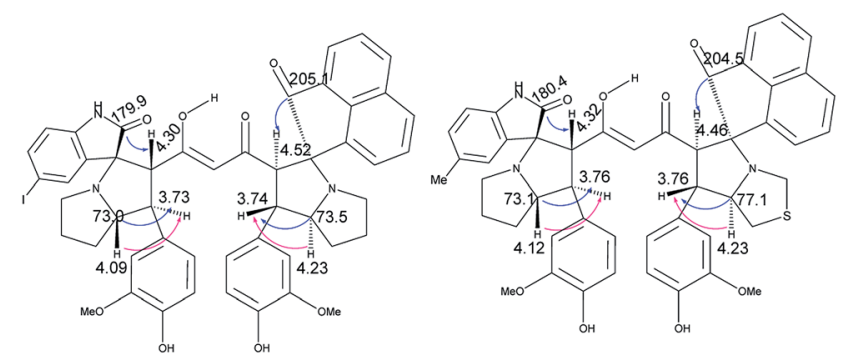

Fig. 3 Important correlations of $7 \mathrm{Bb}$ and $7 \mathrm{Db}[\mathrm{HMBC} \rightarrow$, $\cos (\rightarrow)]$.

The products were again characterized from detailed spectral studies. The product $( \pm)-7 \mathbf{B}$ showed peak at $\mathrm{m} / \mathrm{z} 930$ ascribed to $[\mathrm{M}+\mathrm{H}]^{+}$in the ESI-Q-TOF MS spectrum. Crucial evidence in support of the proposed structure came from the observed HMBC correlation in the 2D NMR spectrum between signals of $\mathrm{C}-10 / 32$, i.e., the oxindole/acenaphthyleno carbonyls $(\delta 179.9 / 205.1)$ and $\mathrm{H}-3 / 25(\delta 4.30 / 4.52)$ and those of $\mathrm{C}-5 / 27$ (i.e., the point of attachment with proline, $\delta$ 73.0/73.5) with $\mathrm{H}-4 / 26$ $(\delta 3.73 / 3.74)$. Further, the COSY relationship between $\mathrm{H}-4 / 26$ $(\delta 3.73 / 3.74)$ and $\mathrm{H}-5 / 27(\delta$ 4.09/4.23) strongly supports the mode of additions (Fig. 3).

\section{Conclusion}

In conclusion, curcumin has been successfully used in a two step sequential 1,3-dipolar azomethine ylide cycloaddition reaction to produce novel mixed bis-pyrrolizidino/thiopyrrolizidino dispiro-oxindolo/acenaphthyleno curcuminoids. The structures of the products were determined by $1 \mathrm{D} / 2 \mathrm{D}$ NMR analysis and MS. The unaltered pharmacophores of curcumin along with the newly generated diversely mixed spiro oxindolo pyrrolizidine moiety might add to the biological effectiveness of the scaffold with increased polarity and solubility.

\section{Experimental section}

\section{General information}

All the compounds evaluated in this work were synthesized in one-pot sequences. Melting points were determined in capillaries and are uncorrected. IR spectra were recorded as $\mathrm{KBr}$ 
pellets using a JASCO 410 FTIR spectrometer. The NMR spectra were recorded using a Bruker 600 DPX spectrometer operating at $600 \mathrm{MHz}$ for ${ }^{1} \mathrm{H}$ and $150 \mathrm{MHz}$ for ${ }^{13} \mathrm{C}$ in pyridine- $d_{5}$ and the chemical shifts are reported in $\delta$ units. Mass spectra (positive mode) were obtained on a ESI-Q-TOF micro mass spectrometer in the electrospray ionization mode.

Curcumin was isolated from Curcuma longa in the usual way. Isatins, acenaphthoquinone and $\alpha$-amino acids were purchased from Alfa-Aesar Company. All other solvents and chromatographic absorbents were procured from E. Merck (Germany) and SRL (India) Ltd. unless otherwise indicated. Thin layer chromatography was performed on pre-coated silica gel $60 \mathrm{~F}_{254}$ aluminum sheets (E. Merck, Germany) using the solvent system $5 \% \mathrm{MeOH}$ in $\mathrm{CHCl}_{3}$ and spots were observed using UV irradiation and iodine. Compounds were separated using AKROS "Automatic TLC Smart Flash” of Yamazen Corporation.

\section{Typical experimental procedure for synthesis of mono cycloaddition products $( \pm)-3$ Aa}

A mixture of 1 (13.6 mmol, $5 \mathrm{~g}$ ), isatin (13.6 mmol, $2.00 \mathrm{~g}$ ) and proline (13.6 mmol, $1.56 \mathrm{~g}$ ) was taken in a round bottom flask, dissolved in $200 \mathrm{~mL}$ methanol, and heated to reflux for specified time period. After completion of the reaction as evident from TLC, the solvent was removed and the crude product was subjected to flash chromatography using increasing concentration of methanol in chloroform as eluant. The product was crystallized from chloroform-methanol mixture (85\%, $6.56 \mathrm{~g})$.

\section{Typical experimental procedure for synthesis of mixed cycloaddition products}

A mixture of $( \pm)$-3Aa (1.76 mmol, $1 \mathrm{~g})$, 5-iodoisatin $(1.76 \mathrm{mmol}$, $481 \mathrm{mg})$ and proline $(1.76 \mathrm{mmol}, 205 \mathrm{mg})$ or thioproline $(1.76 \mathrm{mmol}, 235 \mathrm{mg})$ was taken in a round bottom flask, dissolved in $50 \mathrm{~mL}$ methanol, and heated to reflux for specified time period. The solvent was removed after completion of the reaction (as evident from TLC), and the crude product was subjected to flash chromatography using increasing concentration of methanol in chloroform as eluant to isolate mixed pyrrolizidino/pyrrilizidino-thiopyrrolizidino dispiro oxindolo curcuminoids. The product was crystallized from chloroformmethanol mixture.

\section{Spectral data of compound 4Aa $( \pm)$}

Obtained as white solid; yield: $20 \%$ (314 mg); $\mathrm{mp}: 219-221{ }^{\circ} \mathrm{C} ; R_{\mathrm{f}}$ 0.49 (6\% $\mathrm{MeOH}$ in $\left.\mathrm{CHCl}_{3}\right)$; UV absorption maximas $\left(\lambda_{\max } \mathrm{nm}\right.$, methanol) 258, 213; IR (KBr, $\left.\nu_{\max } \mathrm{cm}^{-1}\right): 3376,2960,2869,1722$, 1612; ${ }^{1} \mathrm{H}$ NMR $\left(P y-d_{5}\right): \delta 11.51(1 \mathrm{H}, \mathrm{s},-\mathrm{NH}), 11.19(1 \mathrm{H}, \mathrm{s},-\mathrm{NH})$, $7.97(1 \mathrm{H}, \mathrm{s}), 7.75(1 \mathrm{H}, \mathrm{d}, J=7.8 \mathrm{~Hz}), 7.38(2 \mathrm{H}, \mathrm{m}), 7.29(2 \mathrm{H}, \mathrm{m})$, $7.22(2 \mathrm{H}, \mathrm{m}), 7.13(1 \mathrm{H}, \mathrm{d}, J=1.2 \mathrm{~Hz}), 6.99(1 \mathrm{H}, \mathrm{t}, J=7.8 \mathrm{~Hz}), 6.96$ $(1 \mathrm{H}, \mathrm{d}, J=6.6 \mathrm{~Hz}), 6.87(1 \mathrm{H}, \mathrm{d}, J=8.4 \mathrm{~Hz}), 6.75(1 \mathrm{H}, \mathrm{dd}, J=1.8$, $8.4 \mathrm{~Hz}), 5.80(1 \mathrm{H}, \mathrm{s}), 4.59(1 \mathrm{H}, \mathrm{d}, J=12.6 \mathrm{~Hz}), 4.43(1 \mathrm{H}, \mathrm{d}, J=$ $12.6 \mathrm{~Hz}), 4.21(2 \mathrm{H}, \mathrm{m}), 3.82(3 \mathrm{H}, \mathrm{s}), 3.75(1 \mathrm{H}, \mathrm{m}), 3.66(3 \mathrm{H}, \mathrm{s})$, $3.64(1 \mathrm{H}, \mathrm{m}), 2.74(2 \mathrm{H}, \mathrm{m}), 2.62(2 \mathrm{H}, \mathrm{m}), 1.87(3 \mathrm{H}, \mathrm{m}), 1.82$ $(2 \mathrm{H}, \mathrm{m}), 1.70(3 \mathrm{H}, \mathrm{m}) .{ }^{13} \mathrm{C}$ NMR $\left(P y-d_{5}\right): \delta 191.8(\mathrm{C}=\mathrm{O}), 190.8$ $(\mathrm{C}=\mathrm{O}), 181.1(\mathrm{C}=\mathrm{O}), 180.2(\mathrm{C}=\mathrm{O}), 149.6(\mathrm{C}), 149.2$ (C), 147.7 (C), 147.6 (C), 143.9 (C), 143.6 (C), $138.9(\mathrm{CH}), 135.8(\mathrm{CH}), 131.9$
(C), 130.9 (C), 129.7 (CH), 129.5 (C), 127.1 (C), 126.9 (CH), 121.7 $(2 \times \mathrm{CH}), 121.0(\mathrm{CH}), 117.4(\mathrm{CH}), 117.1(\mathrm{CH}), 113.2(\mathrm{CH}), 111.7$ (CH), $111.4(\mathrm{CH}), 110.6(\mathrm{CH}), 101.4(\mathrm{CH}), 84.7$ (C), $74.49(\mathrm{C})$, $74.46(\mathrm{C}), 74.42(\mathrm{CH}), 73.5(\mathrm{CH}), 65.2(\mathrm{CH}), 63.6(\mathrm{CH}), 56.3$ (OMe), $56.1(\mathrm{OMe}), 52.8(\mathrm{CH}), 52.6(\mathrm{CH}), 48.6\left(\mathrm{CH}_{2}\right), 48.3\left(\mathrm{CH}_{2}\right)$, $31.13\left(\mathrm{CH}_{2}\right), 31.06\left(\mathrm{CH}_{2}\right), 28.1\left(\mathrm{CH}_{2}\right), 27.8\left(\mathrm{CH}_{2}\right)$. MS [ESI-MS, positive mode]: found $m / z 895[\mathrm{M}+\mathrm{H}]^{+}$. HRMS [ESI-MS, positive mode]: MF: $\mathrm{C}_{45} \mathrm{H}_{43} \mathrm{~N}_{4} \mathrm{O}_{8} \mathrm{I}$; found $m / z$ 917.2042 $[\mathrm{M}+\mathrm{Na}]^{+}$ [calcd. 917.2023].

\section{Spectral data of compound $4 \mathrm{Ab}( \pm)$}

Obtained as white solid; yield: $23 \%$ (362 mg); mp: $192-194{ }^{\circ} \mathrm{C} ; R_{\mathrm{f}}$ $0.43\left(6 \% \mathrm{MeOH}\right.$ in $\left.\mathrm{CHCl}_{3}\right)$; UV absorption maximas $\left(\lambda_{\max } \mathrm{nm}\right.$, methanol) 201; IR (KBr, $\left.\nu_{\max } \mathrm{cm}^{-1}\right): 3383,2960,2872,1718$, 1611. ${ }^{1} \mathrm{H}$ NMR $\left(P y-d_{5}\right): \delta 12.18(1 \mathrm{H}, \mathrm{s},-\mathrm{NH}), 11.81(1 \mathrm{H}, \mathrm{s},-\mathrm{NH})$, $7.91(1 \mathrm{H}, \mathrm{d}, J=1.8 \mathrm{~Hz}), 7.63(1 \mathrm{H}, \mathrm{dd}, J=1.8,8.4 \mathrm{~Hz}), 7.25(2 \mathrm{H}$, $\mathrm{m}), 7.19(1 \mathrm{H}, \mathrm{m}), 7.17(2 \mathrm{H}, \mathrm{d}, J=7.8 \mathrm{~Hz}), 7.07(1 \mathrm{H}, \mathrm{d}, J=1.8 \mathrm{~Hz})$, $7.00(2 \mathrm{H}, \mathrm{m}), 6.92(1 \mathrm{H}, \mathrm{d}, J=7.2 \mathrm{~Hz}), 6.85(2 \mathrm{H}, \mathrm{m}), 5.94(1 \mathrm{H}, \mathrm{s})$, $4.42(2 \mathrm{H}, \mathrm{t}, J=12.0 \mathrm{~Hz}), 4.20(1 \mathrm{H}, \mathrm{m}), 4.10(1 \mathrm{H}, \mathrm{m}), 3.85(1 \mathrm{H}, \mathrm{m})$, $3.79(3 \mathrm{H}, \mathrm{s}), 3.70(1 \mathrm{H}, \mathrm{m}), 3.67(3 \mathrm{H}, \mathrm{s}), 2.69(2 \mathrm{H}, \mathrm{m}), 2.61$ $(2 \mathrm{H}, \mathrm{m}), 1.86(2 \mathrm{H}, \mathrm{m}), 1.79(1 \mathrm{H}, \mathrm{m}), 1.69(5 \mathrm{H}, \mathrm{m}) .{ }^{13} \mathrm{C} \mathrm{NMR}$ $\left(P y-d_{5}\right): \delta 191.6(\mathrm{C}=\mathrm{O}), 189.7(\mathrm{C}=\mathrm{O}), 181.2(\mathrm{C}=\mathrm{O}), 180.5(\mathrm{C}=$ O), 149.2 (C), 149.1 (C), 147.52 (C), 147.50 (C), 143.9 (C), 143.5 (C), $138.8(\mathrm{CH}), 135.8(\mathrm{CH}), 131.4(\mathrm{C}), 131.2(\mathrm{C}), 129.8(\mathrm{CH})$, $129.5(\mathrm{C}), 127.1(\mathrm{CH}), 126.6(\mathrm{C}), 121.8(\mathrm{CH}), 120.7(2 \times \mathrm{CH})$, $117.2(\mathrm{CH}), 117.0(\mathrm{CH}), 113.0(\mathrm{CH}), 112.2(\mathrm{CH}), 112.1(\mathrm{CH}), 110.6$ $(\mathrm{CH}), 101.3(\mathrm{CH}), 84.7(\mathrm{C}), 74.9(2 \times \mathrm{C}), 73.7(\mathrm{CH}), 73.6(\mathrm{CH})$, $65.1(\mathrm{CH}), 64.3(\mathrm{CH}), 56.2$ (OMe), 56.1 (OMe), $52.5(\mathrm{CH}), 51.8$ $(\mathrm{CH}), 48.5\left(\mathrm{CH}_{2}\right), 48.2\left(\mathrm{CH}_{2}\right), 31.4\left(\mathrm{CH}_{2}\right), 31.3\left(\mathrm{CH}_{2}\right), 28.4\left(\mathrm{CH}_{2}\right)$, $28.0\left(\mathrm{CH}_{2}\right)$. MS [ESI-MS, positive mode]: found $m / z 895[\mathrm{M}+\mathrm{H}]^{+}$, $917[\mathrm{M}+\mathrm{Na}]^{+}$. HRMS [ESI-MS, positive mode]: MF: $\mathrm{C}_{45} \mathrm{H}_{43} \mathrm{~N}_{4} \mathrm{O}_{8} \mathrm{I}$; found $m / z$ 917.2034 [M + Na $]^{+}[$calcd. 917.2023].

\section{Spectral data of compound 4Ac $( \pm)$}

Obtained as white solid; yield: $21 \%$ (330 mg); mp: $175-177^{\circ} \mathrm{C} ; R_{\mathrm{f}}$ 0.39 (6\% $\mathrm{MeOH}$ in $\mathrm{CHCl}_{3}$ ); UV absorption maximas $\left(\lambda_{\max } \mathrm{nm}\right.$, methanol) 283, 231; ${ }^{1} \mathrm{H}$ NMR $\left(P y-d_{5}\right): \delta 11.93(1 \mathrm{H}, \mathrm{s},-\mathrm{NH}), 11.54$ $(1 \mathrm{H}, \mathrm{s},-\mathrm{NH}), 8.26(1 \mathrm{H}, \mathrm{s}), 7.60(1 \mathrm{H}, \mathrm{m}), 7.35(1 \mathrm{H}, \mathrm{s}), 7.28(1 \mathrm{H}, \mathrm{t}$, $J=7.8 \mathrm{~Hz}), 7.23(2 \mathrm{H}, \mathrm{m}), 7.16(1 \mathrm{H}, \mathrm{m}), 7.05(2 \mathrm{H}, \mathrm{m}), 6.93(1 \mathrm{H}, \mathrm{d}$, $J=8.4 \mathrm{~Hz}), 6.89(2 \mathrm{H}, \mathrm{m}), 6.54(1 \mathrm{H}, \mathrm{d}, J=7.8 \mathrm{~Hz}), 6.05(1 \mathrm{H}, \mathrm{s})$, $4.71(1 \mathrm{H}, \mathrm{m}), 4.62(1 \mathrm{H}, \mathrm{d}, J=12 \mathrm{~Hz}), 4.33(1 \mathrm{H}, \mathrm{m}), 4.11(1 \mathrm{H}, \mathrm{d}$, $J=12 \mathrm{~Hz}), 3.95(1 \mathrm{H}, \mathrm{m}), 3.71(3 \mathrm{H}, \mathrm{s}), 3.55(3 \mathrm{H}, \mathrm{s}), 3.23(1 \mathrm{H}, \mathrm{m})$, $2.83(2 \mathrm{H}, \mathrm{m}), 1.96(1 \mathrm{H}, \mathrm{m}), 1.90(2 \mathrm{H}, \mathrm{m}), 1.74(2 \mathrm{H}, \mathrm{m}), 1.64(2 \mathrm{H}$, $\mathrm{m}), 1.40(2 \mathrm{H}, \mathrm{m}) .{ }^{13} \mathrm{C}$ NMR $\left(P y-d_{5}\right): \delta 196.3(\mathrm{C}=\mathrm{O}), 194.5(\mathrm{C}=\mathrm{O})$, $181.2(\mathrm{C}=\mathrm{O}), 179.4(\mathrm{C}=\mathrm{O}), 149.3(\mathrm{C}), 148.3(\mathrm{C}), 147.7(\mathrm{C}), 147.5$ (C), 144.6 (C), $144.1(\mathrm{C}), 138.7(\mathrm{CH}), 136.2(\mathrm{CH}), 131.4(\mathrm{C}), 131.0$ (C), $129.9(\mathrm{CH}), 127.7(\mathrm{CH}), 126.9(\mathrm{C}), 126.8(\mathrm{C}), 124.1(\mathrm{CH})$, $121.7(\mathrm{CH}), 121.4(\mathrm{CH}), 117.1(\mathrm{CH}), 116.5(\mathrm{CH}), 113.0(\mathrm{CH}), 112.6$ $(\mathrm{CH}), 112.3(\mathrm{CH}), 110.7(\mathrm{CH}), 102.9(\mathrm{CH}), 84.3(\mathrm{C}), 76.5(\mathrm{C}), 75.1$ (C), $73.8(\mathrm{CH}), 65.7(\mathrm{CH}), 64.6(\mathrm{CH}), 56.1(\mathrm{OMe}), 55.7(\mathrm{OMe}), 53.3$ $(\mathrm{CH}), 52.7(\mathrm{CH}), 52.0(\mathrm{CH}), 51.5\left(\mathrm{CH}_{2}\right), 48.5\left(\mathrm{CH}_{2}\right), 31.3\left(\mathrm{CH}_{2}\right)$, $29.9\left(\mathrm{CH}_{2}\right), 28.2\left(\mathrm{CH}_{2}\right), 26.8\left(\mathrm{CH}_{2}\right)$. MS [ESI-MS, positive mode]: found $m / z 895[\mathrm{M}+\mathrm{H}]^{+}, 917[\mathrm{M}+\mathrm{Na}]^{+}$. HRMS [ESI-MS, positive mode]: $\mathrm{MF}: \mathrm{C}_{45} \mathrm{H}_{43} \mathrm{~N}_{4} \mathrm{O}_{8} \mathrm{I}$; found $m / z$ 917.2017 [M $\left.+\mathrm{Na}\right]^{+}$[calcd. 917.2023]. 


\section{Spectral data of compound 4Ad $( \pm)$}

Obtained as white solid; yield: $24 \%(378 \mathrm{mg}) ; \mathrm{mp}: 218-220^{\circ} \mathrm{C} ; R_{\mathrm{f}}$ 0.38 (6\% MeOH in $\left.\mathrm{CHCl}_{3}\right)$; UV absorption maximas $\left(\lambda_{\max } \mathrm{nm}\right.$, methanol) 262, 212; IR (KBr, $\left.\nu_{\max } \mathrm{cm}^{-1}\right): 3391,2960,1720,1613$; ${ }^{1} \mathrm{H}$ NMR $\left(P y-d_{5}\right): \delta 11.77(1 \mathrm{H}, \mathrm{s},-\mathrm{NH}), 11.51(1 \mathrm{H}, \mathrm{s},-\mathrm{NH}), 8.33$ $(1 \mathrm{H}, \mathrm{s}), 7.67(1 \mathrm{H}, \mathrm{d}, J=7.2 \mathrm{~Hz}), 7.60(1 \mathrm{H}, \mathrm{d}, J=8.4 \mathrm{~Hz}), 7.36(1 \mathrm{H}$, s), $7.30(1 \mathrm{H}, \mathrm{t}, J=7.8 \mathrm{~Hz}), 7.20(1 \mathrm{H}, \mathrm{m}), 7.15(2 \mathrm{H}, \mathrm{m}), 7.01(1 \mathrm{H}, \mathrm{d}$, $J=7.8 \mathrm{~Hz}), 6.97(1 \mathrm{H}, \mathrm{m}), 6.92(1 \mathrm{H}, \mathrm{m}), 6.89(1 \mathrm{H}, \mathrm{s}), 6.54(1 \mathrm{H}, \mathrm{d}$, $J=8.4 \mathrm{~Hz}), 5.97(1 \mathrm{H}, \mathrm{s}), 4.96(1 \mathrm{H}, \mathrm{m}), 4.67(1 \mathrm{H}, \mathrm{q}, J=8.4 \mathrm{~Hz})$, $4.59(1 \mathrm{H}, \mathrm{d}, J=12.6 \mathrm{~Hz}), 4.37(1 \mathrm{H}, \mathrm{m}), 4.23(1 \mathrm{H}, \mathrm{d}, J=12.0 \mathrm{~Hz})$, $3.98(1 \mathrm{H}, \mathrm{m}), 3.70(3 \mathrm{H}, \mathrm{s}), 3.56(3 \mathrm{H}, \mathrm{s}), 3.15(1 \mathrm{H}, \mathrm{m}), 2.88(1 \mathrm{H}$, $\mathrm{m}), 2.74(2 \mathrm{H}, \mathrm{m}), 1.96(1 \mathrm{H}, \mathrm{m}), 1.89(2 \mathrm{H}, \mathrm{m}), 1.77(1 \mathrm{H}, \mathrm{m}), 1.67$ $(1 \mathrm{H}, \mathrm{m}), 1.61(1 \mathrm{H}, \mathrm{m}), 1.51(1 \mathrm{H}, \mathrm{m}), 1.23(1 \mathrm{H}, \mathrm{m}) .{ }^{13} \mathrm{C} \mathrm{NMR}(P y-$ $\left.d_{5}\right): \delta 197.7(\mathrm{C}=\mathrm{O}), 186.0(\mathrm{C}=\mathrm{O}), 181.2(\mathrm{C}=\mathrm{O}), 179.5(\mathrm{C}=\mathrm{O})$, 149.3 (C), 148.3 (C), 147.7 (C), 147.5 (C), 144.6 (C), 144.0 (C), $138.7(\mathrm{CH}), 136.0(\mathrm{CH}), 131.5(\mathrm{C}), 131.0(\mathrm{C}), 129.9(\mathrm{CH}), 128.0$ (CH), 127.2 (C), $126.9(\mathrm{C}), 121.7(\mathrm{CH}), 121.4(\mathrm{CH}), 121.3(\mathrm{CH})$, $117.1(\mathrm{CH}), 116.5(\mathrm{CH}), 113.3(\mathrm{CH}), 112.6(\mathrm{CH}), 112.4(\mathrm{CH}), 110.8$ $(\mathrm{CH}), 102.9(\mathrm{CH}), 84.3(\mathrm{C}), 76.4(\mathrm{C}), 75.4(\mathrm{C}), 73.7(\mathrm{CH}), 65.6$ (CH), $64.5(\mathrm{CH}), 56.1(\mathrm{OMe}), 55.8(\mathrm{OMe}), 53.8(\mathrm{CH}), 52.6(\mathrm{CH})$, $51.5\left(\mathrm{CH}_{2}\right), 51.1(\mathrm{CH}), 48.6\left(\mathrm{CH}_{2}\right), 31.3\left(\mathrm{CH}_{2}\right), 29.4\left(\mathrm{CH}_{2}\right), 28.2$ $\left(\mathrm{CH}_{2}\right), 26.8\left(\mathrm{CH}_{2}\right)$. MS [ESI-MS, positive mode]: found $\mathrm{m} / \mathrm{z} 895$ $[\mathrm{M}+\mathrm{H}]^{+}, 917[\mathrm{M}+\mathrm{Na}]^{+}$. HRMS [ESI-MS, positive mode]: MF: $\mathrm{C}_{45} \mathrm{H}_{43} \mathrm{~N}_{4} \mathrm{O}_{8} \mathrm{I}$; found $\mathrm{m} / z$ 895.2209 [M $\left.+\mathrm{H}\right]^{+}$[calcd. 895.2204].

\section{Spectral data of compound 4Ba $( \pm)$}

Obtained as white solid; yield: $19 \%(266 \mathrm{mg})$; mp: $172-174{ }^{\circ} \mathrm{C} ; R_{\mathrm{f}}$ $0.50\left(6 \% \mathrm{MeOH}\right.$ in $\left.\mathrm{CHCl}_{3}\right)$; UV absorption maximas $\left(\lambda_{\max } \mathrm{nm}\right.$, methanol) 283,$231 ;{ }^{1} \mathrm{H}$ NMR $\left(P y-d_{5}\right): \delta 10.94(1 \mathrm{H}, \mathrm{s},-\mathrm{NH}), 10.91$ $(1 \mathrm{H}, \mathrm{s},-\mathrm{NH}), 7.31(1 \mathrm{H}, \mathrm{d}, J=7.8 \mathrm{~Hz}), 7.27(3 \mathrm{H}, \mathrm{m}), 7.21(1 \mathrm{H}, \mathrm{s})$, $7.18(2 \mathrm{H}, \mathrm{m}), 7.12(1 \mathrm{H}, \mathrm{d}, J=7.8 \mathrm{~Hz}), 6.92(2 \mathrm{H}, \mathrm{m}), 6.88(1 \mathrm{H}, \mathrm{d}$, $J=7.8 \mathrm{~Hz}), 6.77(1 \mathrm{H}, \mathrm{s}), 5.77(1 \mathrm{H}, \mathrm{s}), 4.50(1 \mathrm{H}, \mathrm{d}, J=12.0 \mathrm{~Hz})$, $4.40(1 \mathrm{H}, \mathrm{d}, J=12.0 \mathrm{~Hz}), 4.31(1 \mathrm{H}, \mathrm{m}), 4.21(1 \mathrm{H}, \mathrm{m}), 3.82(1 \mathrm{H}$, $\mathrm{m}), 3.77(3 \mathrm{H}, \mathrm{s}), 3.74(1 \mathrm{H}, \mathrm{m}), 3.71(3 \mathrm{H}, \mathrm{s}), 2.80(2 \mathrm{H}, \mathrm{m}), 2.65$ $(1 \mathrm{H}, \mathrm{m}), 2.60(1 \mathrm{H}, \mathrm{m}), 2.26(3 \mathrm{H}, \mathrm{s}), 2.22(3 \mathrm{H}, \mathrm{s}), 1.86(4 \mathrm{H}, \mathrm{m})$, $1.78(2 \mathrm{H}, \mathrm{m}), 1.68(2 \mathrm{H}, \mathrm{m}) .{ }^{13} \mathrm{C}$ NMR $\left(P y-d_{5}\right): \delta 192.1(\mathrm{C}=\mathrm{O})$, $190.3(\mathrm{C}=\mathrm{O}), 181.3(\mathrm{C}=\mathrm{O}), 181.1(\mathrm{C}=\mathrm{O}), 149.24(\mathrm{C}), 149.18$ (C), 147.5 (C), 147.4 (C), 143.5 (C), 139.9 (C), 131.9 (CH), 131.8 (C), 131.5 (C), 130.6 (C), $129.7(\mathrm{CH}), 127.3(\mathrm{CH}), 126.6(\mathrm{C})$, $126.2(\mathrm{C}), 125.5(\mathrm{CH}), 121.6(\mathrm{CH}), 121.13(\mathrm{CH}), 121.07(\mathrm{CH})$, $119.4(\mathrm{C}), 117.2(\mathrm{CH}), 117.1(\mathrm{CH}), 112.2(\mathrm{CH}), 111.8(\mathrm{CH}), 110.6$ (CH), 101.6 (CH), 74.9 (C), 74.5 (C), 73.7 (CH), $73.5(\mathrm{CH}), 65.1$ (CH), $64.2(\mathrm{CH}), 56.2(\mathrm{OMe}), 56.1(\mathrm{OMe}), 52.53(\mathrm{CH}), 52.50(\mathrm{CH})$, $48.6\left(\mathrm{CH}_{2}\right), 48.4\left(\mathrm{CH}_{2}\right), 31.2\left(\mathrm{CH}_{2}\right), 30.9\left(\mathrm{CH}_{2}\right), 28.1\left(\mathrm{CH}_{2}\right), 27.6$ $\left(\mathrm{CH}_{2}\right), 21.2\left(\mathrm{CH}_{3}\right), 17.2\left(\mathrm{CH}_{3}\right)$. MS [ESI-MS, positive mode]: found $m / z 797[\mathrm{M}+\mathrm{H}]^{+}$. HRMS [ESI-MS, positive mode]: MF: $\mathrm{C}_{47} \mathrm{H}_{48} \mathrm{~N}_{4} \mathrm{O}_{8}$; found $m / z$ 819.3361 [M + Na] $]^{+}$[calcd. 819.3370].

\section{Spectral data of compound $\mathbf{4 B b}( \pm)$}

Obtained as white solid; yield: $22 \%(308 \mathrm{mg}) ; \mathrm{mp}: 188-190{ }^{\circ} \mathrm{C} ; R_{\mathrm{f}}$ $0.44\left(6 \% \mathrm{MeOH}\right.$ in $\left.\mathrm{CHCl}_{3}\right)$; UV absorption maximas $\left(\lambda_{\max } \mathrm{nm}\right.$, methanol) 296,$231 ;{ }^{1} \mathrm{H}$ NMR $\left(P y-d_{5}\right): \delta 11.85(1 \mathrm{H}, \mathrm{s},-\mathrm{NH}), 11.73$ $(1 \mathrm{H}, \mathrm{s},-\mathrm{NH}), 7.25(2 \mathrm{H}, \mathrm{m}), 7.21(1 \mathrm{H}, \mathrm{m}), 7.18(2 \mathrm{H}, \mathrm{d}, J=7.8 \mathrm{~Hz})$, $7.11(2 \mathrm{H}, \mathrm{m}), 7.03(1 \mathrm{H}, \mathrm{d}, J=7.8 \mathrm{~Hz}), 6.97(1 \mathrm{H}, \mathrm{d}, J=7.8 \mathrm{~Hz})$, $6.91(2 \mathrm{H}, \mathrm{m}), 6.75(1 \mathrm{H}, \mathrm{s}), 6.00(1 \mathrm{H}, \mathrm{s}), 4.48(1 \mathrm{H}, \mathrm{d}, J=12.0$
$\mathrm{Hz}), 4.41(1 \mathrm{H}, \mathrm{d}, J=12.0 \mathrm{~Hz}), 4.18(2 \mathrm{H}, \mathrm{m}), 3.87(1 \mathrm{H}, \mathrm{m}), 3.74$ $(3 \mathrm{H}, \mathrm{s}), 3.71(3 \mathrm{H}, \mathrm{s}), 3.66(1 \mathrm{H}, \mathrm{m}), 2.80(1 \mathrm{H}, \mathrm{m}), 2.73(1 \mathrm{H}, \mathrm{m})$, $2.66(1 \mathrm{H}, \mathrm{m}), 2.61(1 \mathrm{H}, \mathrm{m}), 2.31(3 \mathrm{H}, \mathrm{s}), 2.16(3 \mathrm{H}, \mathrm{s}), 1.90$ $(1 \mathrm{H}, \mathrm{m}), 1.83(3 \mathrm{H}, \mathrm{m}), 1.71(3 \mathrm{H}, \mathrm{m}), 1.63(1 \mathrm{H}, \mathrm{m}){ }^{13} \mathrm{C} \mathrm{NMR}(P y-$ $\left.d_{5}\right): \delta 191.7(\mathrm{C}=\mathrm{O}), 190.1(\mathrm{C}=\mathrm{O}), 181.6(\mathrm{C}=\mathrm{O}), 181.2(\mathrm{C}=\mathrm{O})$, 149.3 (C), 149.2 (C), 147.63 (C), 147.57 (C), 143.9 (C), 140.0 (C), 131.9 (CH), 131.7 (C), 131.5 (C), 130.7 (C), $129.8(\mathrm{CH}), 127.1$ $(\mathrm{CH}), 126.7(\mathrm{C}), 126.4(\mathrm{C}), 125.3(\mathrm{CH}), 121.6(\mathrm{CH}), 120.9(\mathrm{CH})$, $120.8(\mathrm{CH}), 119.4(\mathrm{C}), 117.1(2 \times \mathrm{CH}), 112.2(\mathrm{CH}), 112.0(\mathrm{CH})$, $110.5(\mathrm{CH}), 101.0(\mathrm{CH}), 75.1(\mathrm{C}), 74.8(\mathrm{C}), 73.8(\mathrm{CH}), 73.7$ $(\mathrm{CH}), 64.7(\mathrm{CH}), 64.2(\mathrm{CH}), 56.12(\mathrm{OMe}), 56.09(\mathrm{OMe}), 52.4(\mathrm{CH})$, $52.2(\mathrm{CH}), 48.6\left(\mathrm{CH}_{2}\right), 48.3\left(\mathrm{CH}_{2}\right), 31.5\left(\mathrm{CH}_{2}\right), 31.2\left(\mathrm{CH}_{2}\right), 28.3$ $\left(\mathrm{CH}_{2}\right), 27.9\left(\mathrm{CH}_{2}\right), 21.4\left(\mathrm{CH}_{3}\right), 17.3\left(\mathrm{CH}_{3}\right)$. MS [ESI-MS, positive mode]: found $m / z 797$ [M $+\mathrm{H}]^{+}$. HRMS [ESI-MS, positive mode]: MF: $\mathrm{C}_{47} \mathrm{H}_{48} \mathrm{~N}_{4} \mathrm{O}_{8}$; found $\mathrm{m} / z$ 819.3351 $[\mathrm{M}+\mathrm{Na}]^{+}$[calcd. 819.3370].

\section{Spectral data of compound $4 \mathrm{Bc}( \pm)$}

Obtained as white solid; yield: $21 \%(294 \mathrm{mg}) ; \mathrm{mp}: 169-171^{\circ} \mathrm{C} ; R_{\mathrm{f}}$ $0.40\left(6 \% \mathrm{MeOH}\right.$ in $\left.\mathrm{CHCl}_{3}\right) ;{ }^{1} \mathrm{H}$ NMR (DMSO- $\left.d_{6}\right): \delta 10.36(1 \mathrm{H}, \mathrm{s}$, $-\mathrm{NH}), 9.93(1 \mathrm{H}, \mathrm{s},-\mathrm{NH}), 8.84(1 \mathrm{H}, \mathrm{d}, J=4.2 \mathrm{~Hz}), 7.46(1 \mathrm{H}, \mathrm{d}, J=$ $7.2 \mathrm{~Hz}), 7.17(1 \mathrm{H}, \mathrm{m}), 7.02(1 \mathrm{H}, \mathrm{s}), 6.95(1 \mathrm{H}, \mathrm{m}), 6.74(4 \mathrm{H}, \mathrm{m})$, $6.41(1 \mathrm{H}, \mathrm{m}), 6.26(1 \mathrm{H}, \mathrm{s}), 6.18(1 \mathrm{H}, \mathrm{d}, J=6.6 \mathrm{~Hz}), 5.62(1 \mathrm{H}, \mathrm{s})$, $4.24(1 \mathrm{H}, \mathrm{m}), 4.02(1 \mathrm{H}, \mathrm{m}), 3.78(3 \mathrm{H}, \mathrm{s}), 3.72(2 \mathrm{H}, \mathrm{m}), 3.56(2 \mathrm{H}$, $\mathrm{m}), 2.97(1 \mathrm{H}, \mathrm{m}), 2.42(2 \mathrm{H}, \mathrm{m}), 2.36(1 \mathrm{H}, \mathrm{m}), 2.25(3 \mathrm{H}, \mathrm{s}), 2.07$ $(3 \mathrm{H}, \mathrm{m}), 1.96(3 \mathrm{H}, \mathrm{s}), 1.82(2 \mathrm{H}, \mathrm{m}), 1.68(3 \mathrm{H}, \mathrm{m}), 1.40(1 \mathrm{H}, \mathrm{m})$, $1.15(1 \mathrm{H}, \mathrm{m}), 0.86(1 \mathrm{H}, \mathrm{m}) .{ }^{13} \mathrm{C}$ NMR (DMSO- $\left.d_{6}\right): \delta 197.2(\mathrm{C}=\mathrm{O})$, 183.6 $(\mathrm{C}=\mathrm{O}), 179.2(\mathrm{C}=\mathrm{O}), 178.7(\mathrm{C}=\mathrm{O}), 147.6(\mathrm{C}), 146.6$ (C), 145.4 (C), 145.1 (C), 142.3 (C), 139.3 (C), 130.8 (CH), 130.3 (C), 129.9 (C), $129.1(\mathrm{CH}), 127.0(\mathrm{CH}), 126.5$ (C), $126.3(\mathrm{C})$, $125.2(\mathrm{C}), 124.6(\mathrm{CH}), 120.7(\mathrm{CH}), 119.9(\mathrm{CH}), 119.7(\mathrm{CH}), 118.2$ (C), $115.6(\mathrm{CH}), 114.9(\mathrm{CH}), 111.8(2 \times \mathrm{CH}), 109.8(\mathrm{CH}), 101.6$ (CH), $74.8(\mathrm{C}), 73.6(\mathrm{C}), 72.5(\mathrm{CH}), 64.1(\mathrm{CH}), 62.3(\mathrm{CH}), 55.6$ (OMe), $54.9(\mathrm{OMe}), 52.6(\mathrm{CH}), 50.4(\mathrm{CH}), 50.3\left(\mathrm{CH}_{2}\right), 48.9(\mathrm{CH})$, $47.4\left(\mathrm{CH}_{2}\right), 30.1\left(\mathrm{CH}_{2}\right), 28.0\left(\mathrm{CH}_{2}\right), 27.2\left(\mathrm{CH}_{2}\right), 25.6\left(\mathrm{CH}_{2}\right), 20.7$ $\left(\mathrm{CH}_{3}\right), 16.2\left(\mathrm{CH}_{3}\right)$. MS [ESI-MS, positive mode]: found $\mathrm{m} / \mathrm{z} 797$ $[\mathrm{M}+\mathrm{H}]^{+}$. HRMS [ESI-MS, positive mode]: MF: $\mathrm{C}_{47} \mathrm{H}_{48} \mathrm{~N}_{4} \mathrm{O}_{8}$; found $m / z$ 819.3395 [M + Na] $]^{+}$[calcd. 819.3370].

\section{Spectral data of compound $4 \mathrm{Bd}( \pm)$}

Obtained as white solid; yield: $23 \%$ (322 mg); mp: $170-172{ }^{\circ} \mathrm{C}$; $R_{\mathrm{f}}$ $0.39\left(6 \% \mathrm{MeOH}\right.$ in $\left.\mathrm{CHCl}_{3}\right)$; UV absorption maximas $\left(\lambda_{\max } \mathrm{nm}\right.$, methanol) 283, 205; IR (KBr, $\left.\nu_{\max } \mathrm{cm}^{-1}\right): 3390,2960,1713,1618$; ${ }^{1} \mathrm{H}$ NMR $\left(P y-d_{5}\right): \delta 11.84(1 \mathrm{H}, \mathrm{s},-\mathrm{NH}), 11.17(1 \mathrm{H}, \mathrm{s},-\mathrm{NH}), 7.69$ $(1 \mathrm{H}, \mathrm{d}, J=7.2 \mathrm{~Hz}), 7.43(1 \mathrm{H}, \mathrm{s}), 7.38(1 \mathrm{H}, \mathrm{s}), 7.31(1 \mathrm{H}, \mathrm{t}, J=$ $7.2 \mathrm{~Hz}), 7.22(1 \mathrm{H}, \mathrm{d}, J=7.2 \mathrm{~Hz}), 7.18(1 \mathrm{H}, \mathrm{d}, J=1.2 \mathrm{~Hz}), 7.16$ $(1 \mathrm{H}, \mathrm{t}, J=7.8 \mathrm{~Hz}), 7.06(1 \mathrm{H}, \mathrm{d}, J=7.8 \mathrm{~Hz}), 6.93(3 \mathrm{H}, \mathrm{m}), 6.81(1 \mathrm{H}$, s), $6.04(1 \mathrm{H}, \mathrm{s}), 4.99(1 \mathrm{H}, \mathrm{m}), 4.73(1 \mathrm{H}, \mathrm{q}, J=7.8 \mathrm{~Hz}), 4.64(1 \mathrm{H}, \mathrm{d}$, $J=6.6 \mathrm{~Hz}), 4.39(1 \mathrm{H}, \mathrm{m}), 4.25(1 \mathrm{H}, \mathrm{d}, J=12 \mathrm{~Hz}), 3.99(1 \mathrm{H}, \mathrm{dd}, J=$ 9.6, $12 \mathrm{~Hz}), 3.75(3 \mathrm{H}, \mathrm{s}), 3.56(3 \mathrm{H}, \mathrm{s}), 3.23(1 \mathrm{H}, \mathrm{m}), 2.86(1 \mathrm{H}, \mathrm{m})$, $2.79(1 \mathrm{H}, \mathrm{t}, J=7.2 \mathrm{~Hz}), 2.73(1 \mathrm{H}, \mathrm{m}), 2.38(3 \mathrm{H}, \mathrm{s}), 2.01(3 \mathrm{H}, \mathrm{s})$, $1.97(1 \mathrm{H}, \mathrm{m}), 1.90(2 \mathrm{H}, \mathrm{m}), 1.76(2 \mathrm{H}, \mathrm{m}), 1.65(1 \mathrm{H}, \mathrm{m}), 1.59$ $(1 \mathrm{H}, \mathrm{m}), 1.30(1 \mathrm{H}, \mathrm{m}) \cdot{ }^{13} \mathrm{C}$ NMR $\left(P y-d_{5}\right): \delta 198.0(\mathrm{C}=\mathrm{O}), 186.2$ $(\mathrm{C}=\mathrm{O}), 181.4(\mathrm{C}=\mathrm{O}), 180.6(\mathrm{C}=\mathrm{O}), 149.3(\mathrm{C}), 148.3(\mathrm{C}), 147.6$ (C), $147.3(\mathrm{C}), 143.9$ (C), $141.0(\mathrm{C}), 131.8(\mathrm{CH}), 131.6(2 \times \mathrm{C})$, 130.9 (C), $130.0(\mathrm{CH}), 128.0$ (CH), 127.9 (C), 126.9 (C), 125.4 
(CH), $121.8(\mathrm{CH}), 121.7(\mathrm{CH}), 121.5(\mathrm{CH}), 119.4(\mathrm{C}), 117.1(\mathrm{CH})$, 116.4 (CH), $113.2(\mathrm{CH}), 112.4(\mathrm{CH}), 110.9(\mathrm{CH}), 102.9(\mathrm{CH}), 76.5$ (C), $75.4(\mathrm{C}), 73.8(\mathrm{CH}), 65.5(\mathrm{CH}), 64.4(\mathrm{CH}), 56.2(\mathrm{OMe}), 55.7$ (OMe), $54.3(\mathrm{CH}), 52.6(\mathrm{CH}), 51.5\left(\mathrm{CH}_{2}\right), 50.9(\mathrm{CH}), 48.6\left(\mathrm{CH}_{2}\right)$, $31.3\left(\mathrm{CH}_{2}\right), 29.4\left(\mathrm{CH}_{2}\right), 28.2\left(\mathrm{CH}_{2}\right), 26.8\left(\mathrm{CH}_{2}\right), 21.4\left(\mathrm{CH}_{3}\right), 17.1$ $\left(\mathrm{CH}_{3}\right)$. MS [ESI-MS, positive mode]: found $\mathrm{m} / z$ $797[\mathrm{M}+\mathrm{H}]^{+}$. HRMS [ESI-MS, positive mode]: MF: $\mathrm{C}_{47} \mathrm{H}_{48} \mathrm{~N}_{4} \mathrm{O}_{8}$; found $\mathrm{m} / \mathrm{z}$ 819.3378 [M $+\mathrm{Na}]^{+}$[calcd. 819.3370].

\section{Spectral data of compound $4 \mathrm{Ca}( \pm)$}

Obtained as white solid; yield: $17 \%$ (230 mg); mp: $183-185{ }^{\circ} \mathrm{C} ; R_{\mathrm{f}}$ 0.48 (6\% MeOH in $\mathrm{CHCl}_{3}$ ); IR ( $\left.\mathrm{KBr}, \nu_{\max } \mathrm{cm}^{-1}\right)$ : 3391, 2961, 2868, 1722, 1615; ${ }^{1} \mathrm{H}$ NMR $\left(P y-d_{5}\right): \delta 11.28(1 \mathrm{H}, \mathrm{s},-\mathrm{NH}), 10.88(1 \mathrm{H}, \mathrm{s}$, $-\mathrm{NH}), 7.59(1 \mathrm{H}, \mathrm{dd}, J=4.8,6.6 \mathrm{~Hz}), 7.34(1 \mathrm{H}, \mathrm{s}), 7.24(2 \mathrm{H}, \mathrm{m}), 7.22$ $(1 \mathrm{H}, \mathrm{m}), 7.18(2 \mathrm{H}, \mathrm{m}), 7.00(3 \mathrm{H}, \mathrm{m}), 6.85(2 \mathrm{H}, \mathrm{m}), 5.70(1 \mathrm{H}, \mathrm{s}), 4.52$ $(1 \mathrm{H}, \mathrm{d}, J=12.0 \mathrm{~Hz}), 4.37(1 \mathrm{H}, \mathrm{d}, J=12.0 \mathrm{~Hz}), 4.29(1 \mathrm{H}, \mathrm{m}), 4.20$ $(1 \mathrm{H}, \mathrm{m}), 3.81(2 \mathrm{H}, \mathrm{m}), 3.73(3 \mathrm{H}, \mathrm{s}), 3.70(3 \mathrm{H}, \mathrm{s}), 2.83(1 \mathrm{H}, \mathrm{m}), 2.77$ $(1 \mathrm{H}, \mathrm{m}), 2.62(2 \mathrm{H}, \mathrm{m}), 2.16(3 \mathrm{H}, \mathrm{s}), 1.85(4 \mathrm{H}, \mathrm{m}), 1.78(2 \mathrm{H}, \mathrm{m}), 1.69$ $(2 \mathrm{H}, \mathrm{m}) \cdot{ }^{13} \mathrm{C}$ NMR $\left(P y-d_{5}\right): \delta 191.2(\mathrm{C}=\mathrm{O}), 190.9(\mathrm{C}=\mathrm{O}), 181.0$ $(\mathrm{C}=\mathrm{O}), 180.8(\mathrm{C}=\mathrm{O}), 149.22(\mathrm{C}), 149.16(\mathrm{C}), 147.5(\mathrm{C}), 147.4(\mathrm{C})$, 142.4 (C), 141.2 (C), 131.6 (C), 131.5 (C), 130.8 (C), $130.2(\mathrm{CH})$, $129.7(\mathrm{CH}), 128.6(\mathrm{C}), 128.0(\mathrm{CH}), 127.5(\mathrm{CH}), 126.7(\mathrm{C}), 126.6(\mathrm{C})$, $120.8(2 \times \mathrm{CH}), 117.1(2 \times \mathrm{CH}), 112.2(\mathrm{CH}), 111.8(\mathrm{CH}), 111.7$ (CH), $110.4(\mathrm{CH}), 101.7(\mathrm{CH}), 74.7(\mathrm{C}), 74.5(\mathrm{C}), 73.7(\mathrm{CH}), 73.2$ $(\mathrm{CH}), 65.1(\mathrm{CH}), 64.0(\mathrm{CH}), 56.1(\mathrm{OMe}), 56.0(\mathrm{OMe}), 52.2(2 \times \mathrm{CH})$, $48.54\left(\mathrm{CH}_{2}\right), 48.51\left(\mathrm{CH}_{2}\right), 31.0\left(\mathrm{CH}_{2}\right), 30.6\left(\mathrm{CH}_{2}\right), 27.9\left(\mathrm{CH}_{2}\right), 27.7$ $\left(\mathrm{CH}_{2}\right), 21.2\left(\mathrm{CH}_{3}\right)$. MS [ESI-MS, positive mode]: found $\mathrm{m} / \mathrm{z} 817[\mathrm{M}+$ $\mathrm{H}]^{+}$. HRMS [ESI-MS, positive mode]: MF: $\mathrm{C}_{46} \mathrm{H}_{45} \mathrm{~N}_{4} \mathrm{O}_{8} \mathrm{Cl}$; found $m / z$ 839.2817 [M + Na $]^{+}$[calcd. 839.2824].

\section{Spectral data of compound $4 \mathrm{Cb}( \pm)$}

Obtained as white solid; yield: $20 \%(271 \mathrm{mg}) ; \mathrm{mp}: 208-210{ }^{\circ} \mathrm{C} ; R_{\mathrm{f}}$ 0.43 (6\% $\mathrm{MeOH}$ in $\left.\mathrm{CHCl}_{3}\right)$; IR $\left(\mathrm{KBr}, \nu_{\max } \mathrm{cm}^{-1}\right)$ : 3382, 1711, 1613, 1516; ${ }^{1} \mathrm{H}$ NMR $\left(P y-d_{5}\right): \delta 12.14(1 \mathrm{H}, \mathrm{s},-\mathrm{NH}), 11.77(1 \mathrm{H}, \mathrm{s}$, $-\mathrm{NH}), 7.59$ (1H, m), $7.35(1 \mathrm{H}, \mathrm{s}), 7.20(1 \mathrm{H}, \mathrm{m}), 7.15(2 \mathrm{H}, \mathrm{d}, J=8.4$ $\mathrm{Hz}), 7.10(1 \mathrm{H}, \mathrm{d}, J=1.8 \mathrm{~Hz}), 7.05(1 \mathrm{H}, \mathrm{d}, J=1.2 \mathrm{~Hz}), 6.94(1 \mathrm{H}, \mathrm{d}$, $J=7.8 \mathrm{~Hz}), 6.86(1 \mathrm{H}, \mathrm{d}, J=7.8 \mathrm{~Hz}), 6.84(1 \mathrm{H}, \mathrm{d}, J=1.2 \mathrm{~Hz}), 6.82$ $(1 \mathrm{H}, \mathrm{d}, J=7.8 \mathrm{~Hz}), 6.80(1 \mathrm{H}, \mathrm{dd}, J=1.2,8.4 \mathrm{~Hz}), 5.92(1 \mathrm{H}, \mathrm{s})$, $4.39(2 \mathrm{H}, \mathrm{d}, J=12.0 \mathrm{~Hz}), 4.20(1 \mathrm{H}, \mathrm{m}), 4.12(1 \mathrm{H}, \mathrm{m}), 3.92(1 \mathrm{H}$, m), $3.82(1 \mathrm{H}, \mathrm{m}), 3.66(3 \mathrm{H}, \mathrm{s}), 3.65(3 \mathrm{H}, \mathrm{s}), 2.82(1 \mathrm{H}, \mathrm{m}), 2.65$ $(3 \mathrm{H}, \mathrm{m}), 2.11(3 \mathrm{H}, \mathrm{s}), 1.85(5 \mathrm{H}, \mathrm{m}), 1.71(3 \mathrm{H}, \mathrm{m}) .{ }^{13} \mathrm{C} \mathrm{NMR}$ $\left(P y-d_{5}\right): \delta 191.2(\mathrm{C}=\mathrm{O}), 189.8(\mathrm{C}=\mathrm{O}), 181.2(\mathrm{C}=\mathrm{O}), 180.9(\mathrm{C}=$ O), $149.0(2 \times \mathrm{C}), 147.42$ (C), 147.36 (C), 142.6 (C), 141.2 (C), 131.6 (C), 131.2 (C), 130.7 (C), $130.3(\mathrm{CH}), 129.9(\mathrm{CH}), 128.7$ (C), $128.2(\mathrm{CH}), 127.5(\mathrm{CH}), 126.7(\mathrm{C}), 126.6(\mathrm{C}), 120.35(\mathrm{CH}), 120.31$ $(\mathrm{CH}), 116.99(\mathrm{CH}), 116.97(\mathrm{CH}), 112.5(\mathrm{CH}), 112.3(\mathrm{CH}), 111.7$ $(\mathrm{CH}), 110.3(\mathrm{CH}), 101.5(\mathrm{CH}), 75.25(\mathrm{C}), 75.22(\mathrm{C}), 73.7(\mathrm{CH}), 73.4$ $(\mathrm{CH}), 65.3(\mathrm{CH}), 64.9(\mathrm{CH}), 56.0(2 \times \mathrm{OMe}), 51.8(\mathrm{CH}), 51.6(\mathrm{CH})$, $48.3\left(\mathrm{CH}_{2}\right), 48.2\left(\mathrm{CH}_{2}\right), 31.4\left(2 \times \mathrm{CH}_{2}\right), 28.4\left(\mathrm{CH}_{2}\right), 28.3\left(\mathrm{CH}_{2}\right)$, $21.3\left(\mathrm{CH}_{3}\right)$. MS [ESI-MS, positive mode]: found $m / z 817[\mathrm{M}+\mathrm{H}]^{+}$, $839[\mathrm{M}+\mathrm{Na}]^{+}$. HRMS [ESI-MS, positive mode]: MF: $\mathrm{C}_{46} \mathrm{H}_{45} \mathrm{~N}_{4} \mathrm{O}_{8} \mathrm{Cl}$; found $m / z$ 839.2820 [M $\left.+\mathrm{Na}\right]^{+}$[calcd. 839.2824].

\section{Spectral data of compound $4 \mathrm{Cc}( \pm)$}

Obtained as white solid; yield: $20 \%(271 \mathrm{mg}) ; \mathrm{mp}: 174-176{ }^{\circ} \mathrm{C} ; R_{\mathrm{f}}$ $0.36\left(6 \% \mathrm{MeOH}\right.$ in $\left.\mathrm{CHCl}_{3}\right) ;{ }^{1} \mathrm{H}$ NMR $\left(P y-d_{5}\right): \delta 12.14(1 \mathrm{H}, \mathrm{s},-\mathrm{NH})$,
$11.20(1 \mathrm{H}, \mathrm{s},-\mathrm{NH}), 7.90(1 \mathrm{H}, \mathrm{d}, J=1.8 \mathrm{~Hz}), 7.53(1 \mathrm{H}, \mathrm{s}), 7.39(1 \mathrm{H}$, $\mathrm{dd}, J=1.8,7.8 \mathrm{~Hz}), 7.30(1 \mathrm{H}, \mathrm{m}), 7.17(1 \mathrm{H}, \mathrm{d}, J=7.8 \mathrm{~Hz}), 7.07$ $(1 \mathrm{H}, \mathrm{d}, J=8.4 \mathrm{~Hz}), 7.04(1 \mathrm{H}, \mathrm{d}, J=8.4 \mathrm{~Hz}), 7.01(1 \mathrm{H}, \mathrm{d}, J=8.4$ $\mathrm{Hz}), 6.91(1 \mathrm{H}, \mathrm{m}), 6.86(2 \mathrm{H}, \mathrm{m}), 6.67(1 \mathrm{H}, \mathrm{d}, J=7.8 \mathrm{~Hz}), 6.21(1 \mathrm{H}$, $\mathrm{s}), 5.04(1 \mathrm{H}, \mathrm{m}), 4.86(1 \mathrm{H}, \mathrm{m}), 4.66(1 \mathrm{H}, \mathrm{d}, J=12.6 \mathrm{~Hz}), 4.31(1 \mathrm{H}$, $\mathrm{m}), 4.25(1 \mathrm{H}, \mathrm{d}, J=12.0 \mathrm{~Hz}), 4.01(1 \mathrm{H}, \mathrm{m}), 3.68(3 \mathrm{H}, \mathrm{s}), 3.51$ $(3 \mathrm{H}, \mathrm{s}), 3.34(1 \mathrm{H}, \mathrm{m}), 2.88(1 \mathrm{H}, \mathrm{m}), 2.82(1 \mathrm{H}, \mathrm{m}), 2.74(1 \mathrm{H}, \mathrm{m})$, $2.34(3 \mathrm{H}, \mathrm{s}), 1.94(3 \mathrm{H}, \mathrm{m}), 1.88(1 \mathrm{H}, \mathrm{m}), 1.78(4 \mathrm{H}, \mathrm{m}) .{ }^{13} \mathrm{C} \mathrm{NMR}$ $\left(P y-d_{5}\right): \delta 197.5(\mathrm{C}=\mathrm{O}), 187.4(\mathrm{C}=\mathrm{O}), 181.0(\mathrm{C}=\mathrm{O}), 180.2(\mathrm{C}=$ O), 149.3 (C), 148.2 (C), 147.7 (C), 147.4 (C), 143.0 (C), 142.4 (C), $131.1(\mathrm{C}), 131.0(\mathrm{C}), 130.2(\mathrm{CH}), 130.0(\mathrm{CH}), 129.2(\mathrm{C}), 128.4(\mathrm{C})$, $128.1(\mathrm{CH}), 127.7(\mathrm{CH}), 127.3(\mathrm{C}), 127.0(\mathrm{C}), 121.6(\mathrm{CH}), 121.2$ $(\mathrm{CH}), 117.2(\mathrm{CH}), 116.4(\mathrm{CH}), 112.9(\mathrm{CH}), 112.4(\mathrm{CH}), 112.0(\mathrm{CH})$, $110.2(\mathrm{CH}), 103.0(\mathrm{CH}), 76.6(\mathrm{C}), 75.2(\mathrm{C}), 74.0(\mathrm{CH}), 65.9(\mathrm{CH})$, $64.6(\mathrm{CH}), 56.1$ (OMe), 55.7 (OMe), $53.9(\mathrm{CH}), 52.4(\mathrm{CH}), 52.1$ $(\mathrm{CH}), 51.5\left(\mathrm{CH}_{2}\right), 48.5\left(\mathrm{CH}_{2}\right), 31.3\left(\mathrm{CH}_{2}\right), 29.1\left(\mathrm{CH}_{2}\right), 28.4\left(\mathrm{CH}_{2}\right)$, $27.0\left(\mathrm{CH}_{2}\right), 21.5\left(\mathrm{CH}_{3}\right)$. MS [ESI-MS, positive mode]: found $\mathrm{m} / \mathrm{z}$ $817[\mathrm{M}+\mathrm{H}]^{+}, 839[\mathrm{M}+\mathrm{Na}]^{+}$. HRMS [ESI-MS, positive mode]: MF: $\mathrm{C}_{46} \mathrm{H}_{45} \mathrm{~N}_{4} \mathrm{O}_{8} \mathrm{Cl}$; found $\mathrm{m} / z$ 839.2839 [M $\left.+\mathrm{Na}\right]^{+}$[calcd. 839.2824].

\section{Spectral data of compound $4 \mathrm{Cd}( \pm)$}

Obtained as white solid; yield: $23 \%(311 \mathrm{mg}) ; \mathrm{mp}: 172-174{ }^{\circ} \mathrm{C} ; R_{\mathrm{f}}$ 0.35 (6\% MeOH in $\left.\mathrm{CHCl}_{3}\right) ;{ }^{1} \mathrm{H}$ NMR $\left(P y-d_{5}\right): \delta 12.20(1 \mathrm{H}, \mathrm{s},-\mathrm{NH})$, 11.24 (1H, s, $-\mathrm{NH}), 7.91$ (1H, s), $7.56(1 \mathrm{H}, \mathrm{m}), 7.39(1 \mathrm{H}, \mathrm{m}), 7.31$ $(1 \mathrm{H}, \mathrm{s}), 7.17(1 \mathrm{H}, \mathrm{m}), 7.07(3 \mathrm{H}, \mathrm{m}), 7.00(1 \mathrm{H}, \mathrm{d}, J=7.2 \mathrm{~Hz}), 6.92$ $(1 \mathrm{H}, \mathrm{m}), 6.86(1 \mathrm{H}, \mathrm{m}), 6.67(1 \mathrm{H}, \mathrm{d}, J=7.8 \mathrm{~Hz}), 6.21(1 \mathrm{H}, \mathrm{s}), 4.87$ $(1 \mathrm{H}, \mathrm{m}), 4.67(1 \mathrm{H}, \mathrm{d}, J=12.6 \mathrm{~Hz}), 4.30(2 \mathrm{H}, \mathrm{m}), 4.03(1 \mathrm{H}, \mathrm{m})$, $3.71(1 \mathrm{H}, \mathrm{m}), 3.68(3 \mathrm{H}, \mathrm{s}), 3.51(3 \mathrm{H}, \mathrm{s}), 2.87(3 \mathrm{H}, \mathrm{m}), 2.34(3 \mathrm{H}, \mathrm{s})$, $2.23(1 \mathrm{H}, \mathrm{s}), 1.92(5 \mathrm{H}, \mathrm{m}), 1.78(3 \mathrm{H}, \mathrm{m}) .{ }^{13} \mathrm{C} \mathrm{NMR}\left(P y-d_{5}\right): \delta 198.4$ $(\mathrm{C}=\mathrm{O}), 185.7(\mathrm{C}=\mathrm{O}), 181.0(\mathrm{C}=\mathrm{O}), 180.1(\mathrm{C}=\mathrm{O}), 149.2(\mathrm{C})$, 148.2 (C), 147.7 (C), 147.4 (C), 142.9 (C), 142.4 (C), 131.3 (C), 130.9 (C), $130.1(\mathrm{CH}), 129.9(\mathrm{CH}), 129.1$ (C), $128.4(\mathrm{C}), 128.1$ $(\mathrm{CH}), 128.0(\mathrm{CH}), 127.6(\mathrm{C}), 126.8(\mathrm{C}), 121.4(\mathrm{CH}), 121.3(\mathrm{CH})$, $117.1(\mathrm{CH}), 116.4(\mathrm{CH}), 113.2(\mathrm{CH}), 112.4(\mathrm{CH}), 111.9(\mathrm{CH}), 110.1$ $(\mathrm{CH}), 103.0(\mathrm{CH}), 76.5(\mathrm{C}), 75.3(\mathrm{C}), 73.7(\mathrm{CH}), 65.5(\mathrm{CH}), 64.7$ (CH), 56.0 (OMe), 55.7 (OMe), $54.0(\mathrm{CH}), 52.4(\mathrm{CH}), 51.5\left(\mathrm{CH}_{2}\right)$, $50.9(\mathrm{CH}), 48.7\left(\mathrm{CH}_{2}\right), 31.0\left(\mathrm{CH}_{2}\right), 29.9\left(\mathrm{CH}_{2}\right), 28.1\left(\mathrm{CH}_{2}\right), 23.2$ $\left(\mathrm{CH}_{2}\right), 21.5\left(\mathrm{CH}_{3}\right) . \mathrm{MS}$ [ESI-MS, positive mode]: found $\mathrm{m} / \mathrm{z} 817$ [M $+\mathrm{H}]^{+}, 839\left[\mathrm{M}+\mathrm{Na}^{+}\right.$. HRMS [ESI-MS, positive mode]: MF: $\mathrm{C}_{46} \mathrm{H}_{45} \mathrm{~N}_{4} \mathrm{O}_{8} \mathrm{Cl}$; found $m / z$ 839.2812 [M $\left.+\mathrm{Na}\right]^{+}$[calcd. 839.2824].

\section{Spectral data of compound $4 \mathrm{Da}( \pm)$}

Obtained as white solid; yield: $18 \%(246 \mathrm{mg}) ; \mathrm{mp}: 238-240{ }^{\circ} \mathrm{C} ; R_{\mathrm{f}}$ 0.47 (6\% $\mathrm{MeOH}$ in $\mathrm{CHCl}_{3}$ ); IR (KBr, $\left.\nu_{\max } \mathrm{cm}^{-1}\right)$ : 3399, 2959, 2868, 1719, 1605; ${ }^{1} \mathrm{H}$ NMR $\left(P y-d_{5}\right): \delta 11.19(1 \mathrm{H}, \mathrm{s},-\mathrm{NH}), 10.89$ $(1 \mathrm{H}, \mathrm{s},-\mathrm{NH}), 7.30(1 \mathrm{H}, \mathrm{dd}, J=2.4 \mathrm{~Hz}), 7.28(1 \mathrm{H}, \mathrm{d}, J=8.4 \mathrm{~Hz})$, $7.25(1 \mathrm{H}, \mathrm{d}, J=7.8 \mathrm{~Hz}), 7.22(1 \mathrm{H}, \mathrm{d}, J=1.8 \mathrm{~Hz}), 7.20(1 \mathrm{H}, \mathrm{d}, J=$ $1.2 \mathrm{~Hz}), 7.14(1 \mathrm{H}, \mathrm{d}, J=1.8 \mathrm{~Hz}), 7.08(1 \mathrm{H}, \mathrm{dd}, J=1.8,8.4 \mathrm{~Hz})$, $7.00(1 \mathrm{H}, \mathrm{td}, J=2.4,8.4 \mathrm{~Hz}), 6.89(3 \mathrm{H}, \mathrm{m}), 6.85(1 \mathrm{H}, \mathrm{q}, J=4.2)$, $5.75(1 \mathrm{H}, \mathrm{s}), 4.49(2 \mathrm{H}, \mathrm{m}), 4.20(2 \mathrm{H}, \mathrm{m}), 3.75(3 \mathrm{H}, \mathrm{s}), 3.72$ $(1 \mathrm{H}, \mathrm{m}), 3.70(3 \mathrm{H}, \mathrm{s}), 3.69(3 \mathrm{H}, \mathrm{s}), 3.68(1 \mathrm{H}, \mathrm{m}), 2.82(1 \mathrm{H}, \mathrm{m})$, $2.73(1 \mathrm{H}, \mathrm{m}), 2.63(2 \mathrm{H}, \mathrm{m}), 1.88(2 \mathrm{H}, \mathrm{m}), 1.78(4 \mathrm{H}, \mathrm{m}), 1.69(2 \mathrm{H}$, $\mathrm{m}) .{ }^{13} \mathrm{C}$ NMR $\left(P y-d_{5}\right): \delta 191.15(\mathrm{C}=\mathrm{O}), 191.11(\mathrm{C}=\mathrm{O}), 180.9(\mathrm{C}=$ O), $180.8(\mathrm{C}=\mathrm{O}), 158.7\left(\mathrm{C},{ }^{1} J_{\mathrm{C}-\mathrm{F}}=237.0 \mathrm{~Hz}\right), 155.4(\mathrm{C}), 149.3(\mathrm{C})$, 149.2 (C), 147.6 (C), 147.5 (C), 139.8 (C), 137.1 (C), 131.5 (C), 131.3 (C), 128.6 (C), $127.9(\mathrm{C}), 121.2(\mathrm{CH}), 121.0(\mathrm{CH}), 117.2$ 
$(\mathrm{CH}), 117.1(\mathrm{CH}), 116.1\left(\mathrm{CH},{ }^{2} J_{\mathrm{C}-\mathrm{F}}=24.0 \mathrm{~Hz}\right), 115.3(\mathrm{CH}), 114.8$ $\left(\mathrm{CH},{ }^{2} J_{\mathrm{C}-\mathrm{F}}=24.0 \mathrm{~Hz}\right), 114.3(\mathrm{CH}), 111.9(\mathrm{CH}), 111.5(\mathrm{CH}), 111.1$ $\left(\mathrm{CH},{ }^{3} J_{\mathrm{C}-\mathrm{F}}=9.0 \mathrm{~Hz}\right), 110.9(\mathrm{CH}), 101.6(\mathrm{CH}), 74.9(\mathrm{C}), 74.8(\mathrm{C})$, $73.8(\mathrm{CH}), 73.5(\mathrm{CH}), 64.6(\mathrm{CH}), 64.5(\mathrm{CH}), 56.2(\mathrm{OMe}), 56.1$ (OMe), 56.0 (OMe), $52.6(\mathrm{CH}), 52.4(\mathrm{CH}), 48.4\left(2 \times \mathrm{CH}_{2}\right), 31.1$ $\left(\mathrm{CH}_{2}\right), 30.9\left(\mathrm{CH}_{2}\right), 28.0\left(\mathrm{CH}_{2}\right), 27.9\left(\mathrm{CH}_{2}\right)$. MS [ESI-MS, positive mode]: found $m / z 817[\mathrm{M}+\mathrm{H}]^{+}, 839[\mathrm{M}+\mathrm{Na}]^{+}$. HRMS [ESI-MS, positive mode]: MF: $\mathrm{C}_{46} \mathrm{H}_{45} \mathrm{~N}_{4} \mathrm{O}_{9} \mathrm{~F}$; found $\mathrm{m} / z$ 839.3084 [M + $\mathrm{Na}]^{+}$[calcd. 839.3068].

\section{Spectral data of compound $4 \mathrm{Db}( \pm)$}

Obtained as white solid; yield: $22 \%(300 \mathrm{mg}) ; \mathrm{mp}: 178-180{ }^{\circ} \mathrm{C} ; R_{\mathrm{f}}$ $0.43\left(6 \% \mathrm{MeOH}\right.$ in $\left.\mathrm{CHCl}_{3}\right)$; IR $\left(\mathrm{KBr}, \nu_{\max } \mathrm{cm}^{-1}\right): 3385,2961$, 2870, 1721, 1605; ${ }^{1} \mathrm{H}$ NMR $\left(P y-d_{5}\right): \delta 11.91(1 \mathrm{H}, \mathrm{s},-\mathrm{NH}), 11.74$ (1H, s, $-\mathrm{NH}), 7.33(1 \mathrm{H}, \mathrm{dd}, J=2.4,8.4 \mathrm{~Hz}), 7.18(2 \mathrm{H}, \mathrm{m}), 7.14$ $(1 \mathrm{H}, \mathrm{d}, J=7.8 \mathrm{~Hz}), 7.11(2 \mathrm{H}, \mathrm{dd}, J=1.8,7.8 \mathrm{~Hz}), 7.01(1 \mathrm{H}, \mathrm{td}, J=$ 2.4, 9.0 Hz), $6.96(1 \mathrm{H}, \mathrm{d}, J=8.4 \mathrm{~Hz}), 6.87(3 \mathrm{H}, \mathrm{m}), 6.83(1 \mathrm{H}, \mathrm{dd}$, $J=2.4,8.4 \mathrm{~Hz}), 5.96(1 \mathrm{H}, \mathrm{s}), 4.44(2 \mathrm{H}, \mathrm{m}), 4.13(2 \mathrm{H}, \mathrm{m}), 3.84(1 \mathrm{H}$, $\mathrm{m}), 3.76(1 \mathrm{H}, \mathrm{m}), 3.73(3 \mathrm{H}, \mathrm{s}), 3.71(3 \mathrm{H}, \mathrm{s}), 3.63(3 \mathrm{H}, \mathrm{s}), 2.76(1 \mathrm{H}$, $\mathrm{m}), 2.65(3 \mathrm{H}, \mathrm{m}), 1.86(2 \mathrm{H}, \mathrm{m}), 1.78(2 \mathrm{H}, \mathrm{m}), 1.69(4 \mathrm{H}, \mathrm{m}) .{ }^{13} \mathrm{C}$ NMR $\left(P y-d_{5}\right): \delta 191.4(\mathrm{C}=\mathrm{O}), 189.5(\mathrm{C}=\mathrm{O}), 181.14(\mathrm{C}=\mathrm{O})$, $181.08(\mathrm{C}=\mathrm{O}), 158.7\left(\mathrm{C},{ }^{1} J_{\mathrm{C}-\mathrm{F}}=237 \mathrm{~Hz}\right), 155.4(\mathrm{C}), 149.1(2 \times \mathrm{C})$, 147.42 (C), 147.40 (C), 139.9 (C), 137.1 (C), 131.5 (C), 131.2 (C), $128.4\left(\mathrm{C},{ }^{3} J_{\mathrm{C}-\mathrm{F}}=7.5 \mathrm{~Hz}\right), 128.0(\mathrm{C}), 120.7(\mathrm{CH}), 120.6(\mathrm{CH})$, $117.1(\mathrm{CH}), 117.0(\mathrm{CH}), 116.2\left(\mathrm{CH},{ }^{2} J_{\mathrm{C}-\mathrm{F}}=24.0 \mathrm{~Hz}\right), 115.4(\mathrm{CH})$, $114.9\left(\mathrm{CH},{ }^{2} J_{\mathrm{C}-\mathrm{F}}=25.5 \mathrm{~Hz}\right), 114.0(\mathrm{CH}), 112.2(\mathrm{CH}), 112.1(\mathrm{CH})$, $111.2\left(\mathrm{CH},{ }^{3} J_{\mathrm{C}-\mathrm{F}}=9 \mathrm{~Hz}\right), 110.9(\mathrm{CH}), 101.5(\mathrm{CH}), 75.4(\mathrm{C}), 75.3$ (C), $73.9(\mathrm{CH}), 73.6(\mathrm{CH}), 65.0(\mathrm{CH}), 64.5(\mathrm{CH}), 56.08(\mathrm{OMe})$, 56.06 (OMe), 55.9 (OMe), $51.9(\mathrm{CH}), 51.8(\mathrm{CH}), 48.2\left(2 \times \mathrm{CH}_{2}\right)$, $31.6\left(\mathrm{CH}_{2}\right), 31.5\left(\mathrm{CH}_{2}\right), 28.41\left(\mathrm{CH}_{2}\right), 28.40\left(\mathrm{CH}_{2}\right) . \mathrm{MS}$ [ESI-MS, positive mode]: found $m / z 817[\mathrm{M}+\mathrm{H}]^{+}, 839[\mathrm{M}+\mathrm{Na}]^{+}$. HRMS [ESI-MS, positive mode]: MF: $\mathrm{C}_{46} \mathrm{H}_{45} \mathrm{~N}_{4} \mathrm{O}_{9} \mathrm{~F}$; found $\mathrm{m} / z 839.3062$ $[\mathrm{M}+\mathrm{Na}]^{+}$[calcd. 839.3068].

\section{Spectral data of compound 4Dc $( \pm)$}

Obtained as white solid; yield: $21 \%$ (286 mg); mp: $196-198{ }^{\circ} \mathrm{C} ; R_{\mathrm{f}}$ 0.37 (6\% MeOH in $\left.\mathrm{CHCl}_{3}\right) ;{ }^{1} \mathrm{H}$ NMR $\left(P y-d_{5}\right): \delta 11.81(1 \mathrm{H}, \mathrm{s},-\mathrm{NH})$, $11.39(1 \mathrm{H}, \mathrm{s},-\mathrm{NH}), 7.66(1 \mathrm{H}, \mathrm{dd}, J=2.4,8.4 \mathrm{~Hz}), 7.40(1 \mathrm{H}, \mathrm{d}, J=$ $1.8 \mathrm{~Hz}), 7.34(1 \mathrm{H}, \mathrm{s}), 7.15(1 \mathrm{H}, \mathrm{d}, J=7.8 \mathrm{~Hz}), 7.09$ (1H, d, $J=9.0$ $\mathrm{Hz}), 7.03$ (1H, m), $7.00(1 \mathrm{H}, \mathrm{dd}, J=2.4,8.4 \mathrm{~Hz}), 6.92(3 \mathrm{H}, \mathrm{m})$, $6.86(1 \mathrm{H}, \mathrm{d}, J=7.8 \mathrm{~Hz}), 6.64(1 \mathrm{H}, \mathrm{q}, J=4.2 \mathrm{~Hz}), 6.11(1 \mathrm{H}, \mathrm{s}), 4.97$ $(1 \mathrm{H}, \mathrm{m}), 4.77(1 \mathrm{H}, \mathrm{m}), 4.66(1 \mathrm{H}, \mathrm{d}, J=12.6 \mathrm{~Hz}), 4.31(1 \mathrm{H}, \mathrm{m})$, $4.19(1 \mathrm{H}, \mathrm{d}, J=12.0 \mathrm{~Hz}), 3.97(1 \mathrm{H}, \mathrm{m}), 3.71(3 \mathrm{H}, \mathrm{s}), 3.68(3 \mathrm{H}, \mathrm{s})$, $3.53(3 \mathrm{H}, \mathrm{s}), 3.25(1 \mathrm{H}, \mathrm{m}), 2.90(1 \mathrm{H}, \mathrm{m}), 2.83(1 \mathrm{H}, \mathrm{m}), 2.74(1 \mathrm{H}$, $\mathrm{m}), 1.93(1 \mathrm{H}, \mathrm{m}), 1.86(3 \mathrm{H}, \mathrm{m}), 1.72(4 \mathrm{H}, \mathrm{m}) .{ }^{13} \mathrm{C} \mathrm{NMR}\left(P y-d_{5}\right)$ : $\delta 195.7(\mathrm{C}=\mathrm{O}), 189.0(\mathrm{C}=\mathrm{O}), 181.2(\mathrm{C}=\mathrm{O}), 180.1(\mathrm{C}=\mathrm{O}), 158.9$ $\left(\mathrm{C},{ }^{1} J_{\mathrm{C}-\mathrm{F}}=235.5 \mathrm{~Hz}\right), 155.6(\mathrm{C}), 149.2(\mathrm{C}), 148.3(\mathrm{C}), 147.64(\mathrm{C})$, 147.56 (C), 140.9 (C), 137.5 (C), 131.5 (C), 129.9 (C, ${ }^{3} J_{\mathrm{C}-\mathrm{F}}=9.4$ $\mathrm{Hz}), 128.4$ (C), $126.9(\mathrm{C}), 121.8(\mathrm{CH}), 121.2(\mathrm{CH}), 117.1(\mathrm{CH})$, $116.5(\mathrm{CH}), 116.1\left(\mathrm{CH},{ }^{2} J_{\mathrm{C}-\mathrm{F}}=22.5 \mathrm{~Hz}\right), 115.7(\mathrm{CH}), 115.2(\mathrm{CH}$, $\left.{ }^{2} J_{\mathrm{C}-\mathrm{F}}=24.0 \mathrm{~Hz}\right), 113.8(\mathrm{CH}), 112.7(\mathrm{CH}), 112.4(\mathrm{CH}), 110.9$ $\left(\mathrm{CH},{ }^{3} J_{\mathrm{C}-\mathrm{F}}=7.5 \mathrm{~Hz}\right), 110.8(\mathrm{CH}), 102.7(\mathrm{CH}), 76.8(\mathrm{C}), 75.4(\mathrm{C})$, $73.9(\mathrm{CH}), 65.8(\mathrm{CH}), 64.7(\mathrm{CH}), 56.1$ (OMe), 56.0 (OMe), 55.7 (OMe), $53.4(\mathrm{CH}), 52.5(\mathrm{CH}), 52.1(\mathrm{CH}), 51.5\left(\mathrm{CH}_{2}\right), 48.5\left(\mathrm{CH}_{2}\right)$, $31.4\left(\mathrm{CH}_{2}\right), 28.9\left(\mathrm{CH}_{2}\right), 28.3\left(\mathrm{CH}_{2}\right), 26.9\left(\mathrm{CH}_{2}\right) . \mathrm{MS}$ [ESI-MS, positive mode]: found $m / z 817[\mathrm{M}+\mathrm{H}]^{+}, 839[\mathrm{M}+\mathrm{Na}]^{+}$. HRMS
[ESI-MS, positive mode]: $\mathrm{MF}: \mathrm{C}_{46} \mathrm{H}_{45} \mathrm{~N}_{4} \mathrm{O}_{9} \mathrm{~F}$; found $\mathrm{m} / z 839.3059$ $[\mathrm{M}+\mathrm{Na}]^{+}$[calcd. 839.3068].

\section{Spectral data of compound $4 \mathrm{Dd}( \pm)$}

Obtained as white solid; yield: $24 \%$ (327 mg); mp: $189-191{ }^{\circ} \mathrm{C} ; R_{\mathrm{f}}$ 0.36 (6\% MeOH in $\left.\mathrm{CHCl}_{3}\right) ;{ }^{1} \mathrm{H}$ NMR $\left(P y-d_{5}\right): \delta 11.66(1 \mathrm{H}, \mathrm{s},-\mathrm{NH})$, $11.37(1 \mathrm{H}, \mathrm{s},-\mathrm{NH}), 7.72(1 \mathrm{H}, \mathrm{d}, J=8.4 \mathrm{~Hz}), 7.45(1 \mathrm{H}, \mathrm{s}), 7.37(1 \mathrm{H}$, s), $7.18(1 \mathrm{H}, \mathrm{d}, J=8.4 \mathrm{~Hz}), 7.10(1 \mathrm{H}, \mathrm{d}, J=7.8 \mathrm{~Hz}), 7.02(2 \mathrm{H}$, $\mathrm{m}), 6.95(3 \mathrm{H}, \mathrm{m}), 6.92(1 \mathrm{H}, \mathrm{m}), 6.66(1 \mathrm{H}, \mathrm{q}, J=3.6 \mathrm{~Hz}), 5.97(1 \mathrm{H}$, s), $4.93(1 \mathrm{H}, \mathrm{t}, J=10.2 \mathrm{~Hz}), 4.71(1 \mathrm{H}, \mathrm{m}), 4.60(1 \mathrm{H}, \mathrm{d}, J=11.4$ $\mathrm{Hz}), 4.41(1 \mathrm{H}, \mathrm{m}), 4.25(1 \mathrm{H}, \mathrm{d}, J=12.0 \mathrm{~Hz}), 4.01(1 \mathrm{H}, \mathrm{m}), 3.80$ $(3 \mathrm{H}, \mathrm{s}), 3.69(3 \mathrm{H}, \mathrm{s}), 3.52(3 \mathrm{H}, \mathrm{s}), 3.20(1 \mathrm{H}, \mathrm{m}), 2.97(1 \mathrm{H}, \mathrm{m}), 2.78$ (2H, m), $1.91(4 \mathrm{H}, \mathrm{m}), 1.77(2 \mathrm{H}, \mathrm{m}), 1.65(1 \mathrm{H}, \mathrm{m}), 1.60(1 \mathrm{H}, \mathrm{m})$. ${ }^{13} \mathrm{C}$ NMR $\left(P y-d_{5}\right): \delta 194.0(\mathrm{C}=\mathrm{O}), 187.2(\mathrm{C}=\mathrm{O}), 181.3(\mathrm{C}=\mathrm{O})$, $180.1(\mathrm{C}=\mathrm{O}), 158.9\left(\mathrm{C},{ }^{1} J_{\mathrm{C}-\mathrm{F}}=235.5 \mathrm{~Hz}\right), 155.6(\mathrm{C}), 149.3(\mathrm{C})$, 148.3 (C), 147.6 (C), 147.5 (C), 140.9 (C), 137.5 (C), 131.7 (C), 130.0 (C), 128.5 (C), $127.4(\mathrm{C}), 121.8(\mathrm{CH}), 121.6(\mathrm{CH}), 117.0$ $(\mathrm{CH}), 116.4(\mathrm{CH}), 116.0\left(\mathrm{CH},{ }^{2} J_{\mathrm{C}-\mathrm{F}}=24.0 \mathrm{~Hz}\right), 115.8(\mathrm{CH}), 115.3$ $\left(\mathrm{CH},{ }^{2} J_{\mathrm{C}-\mathrm{F}}=24.0 \mathrm{~Hz}\right), 114.3(\mathrm{CH}), 113.1(\mathrm{CH}), 112.3(\mathrm{CH}), 110.9$ $(\mathrm{CH}), 110.8(\mathrm{CH}), 102.8(\mathrm{CH}), 76.8(\mathrm{C}), 75.7(\mathrm{C}), 73.6(\mathrm{CH}), 65.8$ (CH), $65.0(\mathrm{CH}), 56.3$ (OMe), 56.1 (OMe), 55.8 (OMe), $53.9(\mathrm{CH})$, $52.7(\mathrm{CH}), 51.45(\mathrm{CH}), 51.41\left(\mathrm{CH}_{2}\right), 48.6\left(\mathrm{CH}_{2}\right), 31.2\left(\mathrm{CH}_{2}\right), 29.3$ $\left(\mathrm{CH}_{2}\right), 28.1\left(\mathrm{CH}_{2}\right), 26.8\left(\mathrm{CH}_{2}\right)$. MS [ESI-MS, positive mode]: found $m / z 817[\mathrm{M}+\mathrm{H}]^{+}, 839[\mathrm{M}+\mathrm{Na}]^{+}$. HRMS [ESI-MS, positive mode]: $\mathrm{MF}$ : $\mathrm{C}_{46} \mathrm{H}_{45} \mathrm{~N}_{4} \mathrm{O}_{9} \mathrm{~F}$; found $m / z$ 839.3059 [M+Na] $]^{+}$calcd. 839.3068].

\section{Spectral data of compound $4 \mathrm{Ea}( \pm)$}

Obtained as white solid; yield: $18 \%(250 \mathrm{mg})$; $\mathrm{mp}: 180-182{ }^{\circ} \mathrm{C} ; R_{\mathrm{f}}$ 0.47 (6\% $\mathrm{MeOH}$ in $\mathrm{CHCl}_{3}$ ); IR (KBr, $\left.\nu_{\max } \mathrm{cm}^{-1}\right)$ : 3397, 2959, 2868, 1720, 1615; ${ }^{1} \mathrm{H}$ NMR $\left(P y-d_{5}\right): \delta 11.02(1 \mathrm{H}, \mathrm{s},-\mathrm{NH}), 10.96$ $(1 \mathrm{H}, \mathrm{s},-\mathrm{NH}), 7.32(1 \mathrm{H}, \mathrm{dd}, J=2.4,8.4 \mathrm{~Hz}), 7.28(2 \mathrm{H}, \mathrm{m}), 7.23$ $(1 \mathrm{H}, \mathrm{d}, J=1.8 \mathrm{~Hz}), 7.21(1 \mathrm{H}, \mathrm{m}), 7.17(1 \mathrm{H}, \mathrm{d}, J=1.2 \mathrm{~Hz})$, 7.09 (1H, dd, $J=1.2,7.8 \mathrm{~Hz}), 6.97(2 \mathrm{H}, \mathrm{m}), 6.81(1 \mathrm{H}, \mathrm{m}), 6.77$ $(1 \mathrm{H}, \mathrm{s}), 5.74(1 \mathrm{H}, \mathrm{s}), 4.52(1 \mathrm{H}, \mathrm{d}, J=12.6 \mathrm{~Hz}), 4.38(1 \mathrm{H}, \mathrm{d}, J=$ $12.0 \mathrm{~Hz}), 4.33(1 \mathrm{H}, \mathrm{m}), 4.17(1 \mathrm{H}, \mathrm{m}), 3.79(2 \mathrm{H}, \mathrm{m}), 3.75(3 \mathrm{H}, \mathrm{s})$, $3.70(3 \mathrm{H}, \mathrm{s}), 2.82(1 \mathrm{H}, \mathrm{m}), 2.76(1 \mathrm{H}, \mathrm{m}), 2.63(2 \mathrm{H}, \mathrm{m}), 2.24(3 \mathrm{H}$, s), $2.20(3 \mathrm{H}, \mathrm{s}), 1.82(6 \mathrm{H}, \mathrm{m}), 1.69(2 \mathrm{H}, \mathrm{m}) .{ }^{13} \mathrm{C}$ NMR $\left(P y-d_{5}\right)$ : $\delta 192.4(\mathrm{C}=\mathrm{O}), 190.2(\mathrm{C}=\mathrm{O}), 181.2(\mathrm{C}=\mathrm{O}), 181.0(\mathrm{C}=\mathrm{O}), 158.7$ $\left(\mathrm{C},{ }^{1} J_{\mathrm{C}-\mathrm{F}}=235.5 \mathrm{~Hz}\right), 149.22(\mathrm{C}), 149.17(\mathrm{C}), 147.5(\mathrm{C}), 147.4(\mathrm{C})$, 139.8 (C), 139.6 (C), 131.9 (CH), 131.7 (C), 131.5 (C), 130.6 (C), $128.3(\mathrm{C}), 126.1(\mathrm{C}), 125.4(\mathrm{CH}), 120.91(\mathrm{CH}), 120.87(\mathrm{CH}), 119.3$ (C), $117.2(\mathrm{CH}), 117.1(\mathrm{CH}), 116.1\left(\mathrm{CH},{ }^{2} J_{\mathrm{C}-\mathrm{F}}=22.5 \mathrm{~Hz}\right), 115.1$ $\left(\mathrm{CH},{ }^{2} J_{\mathrm{C}-\mathrm{F}}=24.0 \mathrm{~Hz}\right), 112.4(\mathrm{CH}), 111.6(\mathrm{CH}), 111.1(\mathrm{CH}), 101.6$ $(\mathrm{CH}), 74.9(\mathrm{C}), 74.8(\mathrm{C}), 73.8(\mathrm{CH}), 73.3(\mathrm{CH}), 65.6(\mathrm{CH}), 63.6$ (CH), 56.1 (OMe), 56.0 (OMe), $52.4(\mathrm{CH}), 52.3(\mathrm{CH}), 48.5\left(\mathrm{CH}_{2}\right)$, $48.4\left(\mathrm{CH}_{2}\right), 31.1\left(\mathrm{CH}_{2}\right), 30.7\left(\mathrm{CH}_{2}\right), 28.0\left(\mathrm{CH}_{2}\right), 27.7\left(\mathrm{CH}_{2}\right), 21.2$ $\left(\mathrm{CH}_{3}\right), 17.1\left(\mathrm{CH}_{3}\right) . \mathrm{MS}$ [ESI-MS, positive mode]: found $\mathrm{m} / \mathrm{z} 815$ $[\mathrm{M}+\mathrm{H}]^{+}, 837[\mathrm{M}+\mathrm{Na}]^{+}$. HRMS [ESI-MS, positive mode]: MF: $\mathrm{C}_{47} \mathrm{H}_{47} \mathrm{~N}_{4} \mathrm{O}_{8} \mathrm{~F}$; found $m / z$ 837.3240 [M $\left.+\mathrm{Na}\right]^{+}$[calcd. 837.3276].

\section{Spectral data of compound $4 \mathrm{~Eb}( \pm)$}

Obtained as white solid; yield: $22 \%$ (305 mg); mp: $182-184{ }^{\circ} \mathrm{C} ; R_{\mathrm{f}}$ 0.43 (6\% $\mathrm{MeOH}$ in $\mathrm{CHCl}_{3}$ ); IR (KBr, $\nu_{\max } \mathrm{cm}^{-1}$ ): 3403, 3208, 2964, 2871, 1713, 1605; ${ }^{1} \mathrm{H}$ NMR $\left(P y-d_{5}\right): \delta 12.03(1 \mathrm{H}, \mathrm{s},-\mathrm{NH})$, $11.73(1 \mathrm{H}, \mathrm{s},-\mathrm{NH}), 7.28(1 \mathrm{H}, \mathrm{d}, J=7.8 \mathrm{~Hz}), 7.17(2 \mathrm{H}, \mathrm{m}), 7.13$ 
(2H, m), $7.08(1 \mathrm{H}, \mathrm{s}), 6.98(1 \mathrm{H}, \mathrm{m}), 6.82(3 \mathrm{H}, \mathrm{m}), 6.69(1 \mathrm{H}, \mathrm{s})$, $5.93(1 \mathrm{H}, \mathrm{s}), 4.38(2 \mathrm{H}, \mathrm{m}), 4.20(1 \mathrm{H}, \mathrm{m}), 4.13(1 \mathrm{H}, \mathrm{m}), 3.92(1 \mathrm{H}$, $\mathrm{m}), 3.78(1 \mathrm{H}, \mathrm{m}), 3.67(3 \mathrm{H}, \mathrm{s}), 3.64(3 \mathrm{H}, \mathrm{s}), 2.84(1 \mathrm{H}, \mathrm{m}), 2.68$ $(3 \mathrm{H}, \mathrm{m}), 2.28(3 \mathrm{H}, \mathrm{s}), 2.12(3 \mathrm{H}, \mathrm{s}), 1.92(1 \mathrm{H}, \mathrm{m}), 1.84(4 \mathrm{H}, \mathrm{m})$, $1.72(3 \mathrm{H}, \mathrm{m}) .{ }^{13} \mathrm{C}$ NMR $\left(P y-d_{5}\right): \delta 190.7(\mathrm{C}=\mathrm{O}), 190.3(\mathrm{C}=\mathrm{O})$, $181.5(\mathrm{C}=\mathrm{O}), 181.3(\mathrm{C}=\mathrm{O}), 158.6\left(\mathrm{C},{ }^{1} J_{\mathrm{C}-\mathrm{F}}=237.0 \mathrm{~Hz}\right), 149.0(2$ $\times \mathrm{C}), 147.44(\mathrm{C}), 147.38(\mathrm{C}), 139.9(2 \times \mathrm{C}), 131.9(\mathrm{CH}), 131.6(\mathrm{C})$, 131.3 (C), 130.6 (C), 128.3 (C), 126.2 (C), $125.5(\mathrm{CH}), 120.3(\mathrm{CH})$, $120.2(\mathrm{CH}), 119.4(\mathrm{C}), 117.0(\mathrm{CH}), 116.9(\mathrm{CH}), 116.2\left(\mathrm{CH},{ }^{2} J_{\mathrm{C}-\mathrm{F}}=\right.$ $24.0 \mathrm{~Hz}), 115.1\left(\mathrm{CH},{ }^{2} J_{\mathrm{C}-\mathrm{F}}=24.0 \mathrm{~Hz}\right), 112.6(\mathrm{CH}), 112.3(\mathrm{CH})$, $111.0\left(\mathrm{CH},{ }^{3} J_{\mathrm{C}-\mathrm{F}}=7.5 \mathrm{~Hz}\right), 101.4(\mathrm{CH}), 75.4(2 \times \mathrm{C}), 73.6(\mathrm{CH})$, $73.4(\mathrm{CH}), 65.2(\mathrm{CH}), 64.9(\mathrm{CH}), 56.04(\mathrm{OMe}), 55.98$ (OMe), 51.8 $(\mathrm{CH}), 51.5(\mathrm{CH}), 48.33\left(\mathrm{CH}_{2}\right), 48.29\left(\mathrm{CH}_{2}\right), 31.5\left(\mathrm{CH}_{2}\right), 31.3\left(\mathrm{CH}_{2}\right)$, $28.4\left(\mathrm{CH}_{2}\right), 28.2\left(\mathrm{CH}_{2}\right), 21.3\left(\mathrm{CH}_{3}\right), 17.2\left(\mathrm{CH}_{3}\right) . \mathrm{MS}$ [ESI-MS, positive mode]: found $m / z 815[\mathrm{M}+\mathrm{H}]^{+}, 837[\mathrm{M}+\mathrm{Na}]^{+}$. HRMS [ESI-MS, positive mode]: $\mathrm{MF}: \mathrm{C}_{47} \mathrm{H}_{47} \mathrm{~N}_{4} \mathrm{O}_{8} \mathrm{~F}$; found $m / z 837.3305$ $[\mathrm{M}+\mathrm{Na}]^{+}$[calcd. 837.3276].

\section{Spectral data of compound $\operatorname{4Ec}( \pm)$}

Obtained as white solid; yield: $21 \%(292 \mathrm{mg}) ; \mathrm{mp}: 172-174{ }^{\circ} \mathrm{C} ; R_{\mathrm{f}}$ 0.38 (6\% MeOH in $\left.\mathrm{CHCl}_{3}\right) ;{ }^{1} \mathrm{H} \mathrm{NMR}\left(P y-d_{5}\right): \delta 12.01(1 \mathrm{H}, \mathrm{s},-\mathrm{NH})$, $11.15(1 \mathrm{H}, \mathrm{s},-\mathrm{NH}), 7.66(1 \mathrm{H}, \mathrm{dd}, J=2.4,8.4 \mathrm{~Hz}), 7.37(1 \mathrm{H}, \mathrm{s})$, $7.31(1 \mathrm{H}, \mathrm{d}, J=1.8 \mathrm{~Hz}), 7.17(1 \mathrm{H}, \mathrm{m}), 7.13(2 \mathrm{H}, \mathrm{m}), 7.01(1 \mathrm{H}, \mathrm{m})$, $6.92(2 \mathrm{H}, \mathrm{m}), 6.85(1 \mathrm{H}, \mathrm{m}), 6.79(1 \mathrm{H}, \mathrm{s}), 6.15(1 \mathrm{H}, \mathrm{s}), 4.99(1 \mathrm{H}$, $\mathrm{dd}, J=9.6,12.0 \mathrm{~Hz}), 4.80(1 \mathrm{H}, \mathrm{q}, J=8.4 \mathrm{~Hz}), 4.66(1 \mathrm{H}, \mathrm{d}, J=12.0$ $\mathrm{Hz}), 4.31(1 \mathrm{H}, \mathrm{m}), 4.23(1 \mathrm{H}, \mathrm{d}, J=12.0 \mathrm{~Hz}), 4.00(1 \mathrm{H}, \mathrm{dd}, J=9.6$, $12.0 \mathrm{~Hz}), 3.70(3 \mathrm{H}, \mathrm{s}), 3.52(3 \mathrm{H}, \mathrm{s}), 3.21(1 \mathrm{H}, \mathrm{m}), 2.83(2 \mathrm{H}, \mathrm{m})$, $2.74(1 \mathrm{H}, \mathrm{m}), 2.33(3 \mathrm{H}, \mathrm{s}), 1.99(3 \mathrm{H}, \mathrm{s}), 1.91(3 \mathrm{H}, \mathrm{m}), 1.83(2 \mathrm{H}$, $\mathrm{m}), 1.76(2 \mathrm{H}, \mathrm{m}), 1.69(1 \mathrm{H}, \mathrm{m}) .{ }^{13} \mathrm{C} \mathrm{NMR}\left(P y-d_{5}\right): \delta 196.8(\mathrm{C}=\mathrm{O})$, $188.2(\mathrm{C}=\mathrm{O}), 181.3(\mathrm{C}=\mathrm{O}), 180.4(\mathrm{C}=\mathrm{O}), 158.9\left(\mathrm{C},{ }^{1} J_{\mathrm{C}-\mathrm{F}}=237.0\right.$ $\mathrm{Hz}$, 149.3 (C), 148.2 (C), 147.7 (C), 147.4 (C), 141.0 (C), 140.2 (C), $131.8(\mathrm{CH}), 131.2(\mathrm{C}), 130.8(\mathrm{C}), 128.8\left(\mathrm{C},{ }^{3} J_{\mathrm{C}-\mathrm{F}}=7.5 \mathrm{~Hz}\right), 127.8$ (C), $127.5(\mathrm{C}), 125.3(\mathrm{CH}), 121.7(\mathrm{CH}), 121.2(\mathrm{CH}), 119.3(\mathrm{C})$, $117.1(\mathrm{CH}), 116.4(\mathrm{CH}), 116.3\left(\mathrm{CH},{ }^{2} J_{\mathrm{C}-\mathrm{F}}=24.0 \mathrm{~Hz}\right), 115.4(\mathrm{CH}$, $\left.{ }^{2} J_{\mathrm{C}-\mathrm{F}}=24.0 \mathrm{~Hz}\right), 113.0(\mathrm{CH}), 112.3(\mathrm{CH}), 111.2\left(\mathrm{CH},{ }^{3} J_{\mathrm{C}-\mathrm{F}}=7.5\right.$ $\mathrm{Hz}), 102.8(\mathrm{CH}), 76.6(\mathrm{C}), 75.4(\mathrm{C}), 73.9(\mathrm{CH}), 65.6(\mathrm{CH}), 64.7$ $(\mathrm{CH}), 56.1(\mathrm{OMe}), 55.6(\mathrm{OMe}), 54.0(\mathrm{CH}), 52.3(\mathrm{CH}), 51.7(\mathrm{CH})$, $51.5\left(\mathrm{CH}_{2}\right), 48.4\left(\mathrm{CH}_{2}\right), 31.3\left(\mathrm{CH}_{2}\right), 29.0\left(\mathrm{CH}_{2}\right), 28.3\left(\mathrm{CH}_{2}\right), 26.9$ $\left(\mathrm{CH}_{2}\right), 21.4\left(\mathrm{CH}_{3}\right), 17.0\left(\mathrm{CH}_{3}\right) . \mathrm{MS}$ [ESI-MS, positive mode]: found $m / z 815[\mathrm{M}+\mathrm{H}]^{+}, 837[\mathrm{M}+\mathrm{Na}]^{+}$. HRMS [ESI-MS, positive mode]: $\mathrm{MF}: \mathrm{C}_{47} \mathrm{H}_{47} \mathrm{~N}_{4} \mathrm{O}_{8} \mathrm{~F}$; found $m / z$ 837.3284 [M+Na $]^{+}$[calcd. 837.3276].

\section{Spectral data of compound $4 \mathrm{Ed}( \pm)$}

Obtained as white solid; yield: $23 \%$ (319 mg); mp: $170-172{ }^{\circ} \mathrm{C} ; R_{\mathrm{f}}$ 0.37 (6\% MeOH in $\left.\mathrm{CHCl}_{3}\right) ;{ }^{1} \mathrm{H}$ NMR $\left(P y-d_{5}\right): \delta 11.87(1 \mathrm{H}, \mathrm{s},-\mathrm{NH})$, $11.14(1 \mathrm{H}, \mathrm{s},-\mathrm{NH}), 7.74(1 \mathrm{H}, \mathrm{dd}, J=2.4,8.4 \mathrm{~Hz}), 7.40(1 \mathrm{H}, \mathrm{s})$, $7.31(1 \mathrm{H}, \mathrm{d}, J=1.8 \mathrm{~Hz}), 7.27(1 \mathrm{H}, \mathrm{m}), 7.18(1 \mathrm{H}, \mathrm{m}), 7.12(2 \mathrm{H}, \mathrm{m})$, $6.95(2 \mathrm{H}, \mathrm{m}), 6.91(1 \mathrm{H}, \mathrm{d}, J=8.4 \mathrm{~Hz}), 6.80(1 \mathrm{H}, \mathrm{s}), 6.04(1 \mathrm{H}, \mathrm{s})$, $5.05(1 \mathrm{H}, \mathrm{dd}, J=9.6,12.0 \mathrm{~Hz}), 4.68(1 \mathrm{H}, \mathrm{m}), 4.61(1 \mathrm{H}, \mathrm{d}, J=12.0$ $\mathrm{Hz}), 4.37$ (1H, m), $4.25(1 \mathrm{H}, \mathrm{d}, J=12.0 \mathrm{~Hz}), 4.00(1 \mathrm{H}, \mathrm{m}), 3.66$ $(3 \mathrm{H}, \mathrm{s}), 3.54(3 \mathrm{H}, \mathrm{s}), 3.29(1 \mathrm{H}, \mathrm{m}), 2.77(2 \mathrm{H}, \mathrm{m}), 2.38(3 \mathrm{H}, \mathrm{s}), 1.99$ $(3 \mathrm{H}, \mathrm{s}), 1.93(2 \mathrm{H}, \mathrm{m}), 1.76(3 \mathrm{H}, \mathrm{m}), 1.64(2 \mathrm{H}, \mathrm{m}), 1.55(2 \mathrm{H}, \mathrm{m})$. ${ }^{13} \mathrm{C}$ NMR $\left(P y-d_{5}\right): \delta 198.0(\mathrm{C}=\mathrm{O}), 186.2(\mathrm{C}=\mathrm{O}), 181.2(\mathrm{C}=\mathrm{O})$, $180.4(\mathrm{C}=\mathrm{O}), 158.9\left(\mathrm{C},{ }^{1} J_{\mathrm{C}-\mathrm{F}}=237.0 \mathrm{~Hz}\right), 149.3(\mathrm{C}), 148.3(\mathrm{C})$, 147.7 (C), 147.4 (C), 141.0 (C), 140.1 (C), 131.8 (CH), 131.2
(C), 130.8 (C), 128.8 (C, ${ }^{3} J_{\mathrm{C}-\mathrm{F}}=7.5 \mathrm{~Hz}$ ), 127.8 (C), 127.3 (C), $125.4(\mathrm{CH}), 121.7(\mathrm{CH}), 121.3(\mathrm{CH}), 119.3(\mathrm{C}), 117.14(\mathrm{CH})$, $117.08(\mathrm{CH}), 116.3\left(\mathrm{CH},{ }^{2} J_{\mathrm{C}-\mathrm{F}}=24.0 \mathrm{~Hz}\right), 115.6\left(\mathrm{CH},{ }^{2} J_{\mathrm{C}-\mathrm{F}}=24.0\right.$ $\mathrm{Hz}), 113.1(\mathrm{CH}), 112.3(\mathrm{CH}), 111.3\left(\mathrm{CH},{ }^{3} J_{\mathrm{C}-\mathrm{F}}=7.5 \mathrm{~Hz}\right), 102.9$ $(\mathrm{CH}), 76.5(\mathrm{C}), 75.7(\mathrm{C}), 73.7(\mathrm{CH}), 65.5(\mathrm{CH}), 64.7(\mathrm{CH}), 56.1$ (OMe), $55.7(\mathrm{OMe}), 54.2(\mathrm{CH}), 52.4(\mathrm{CH}), 51.5\left(\mathrm{CH}_{2}\right), 50.8(\mathrm{CH})$, $48.5\left(\mathrm{CH}_{2}\right), 31.2\left(\mathrm{CH}_{2}\right), 29.9\left(\mathrm{CH}_{2}\right), 28.3\left(\mathrm{CH}_{2}\right), 26.8\left(\mathrm{CH}_{2}\right), 21.4$ $\left(\mathrm{CH}_{3}\right), 17.0\left(\mathrm{CH}_{3}\right)$. MS [ESI-MS, positive mode]: found $\mathrm{m} / \mathrm{z} 815[\mathrm{M}$ $+\mathrm{H}]^{+}, 837[\mathrm{M}+\mathrm{Na}]^{+}$. HRMS [ESI-MS, positive mode]: MF: $\mathrm{C}_{47} \mathrm{H}_{47} \mathrm{~N}_{4} \mathrm{O}_{8} \mathrm{~F}$; found $m / z$ 837.3264 [M $\left.+\mathrm{Na}\right]^{+}$[calcd. 837.3276].

\section{Spectral data of compound $4 \mathrm{Fa}( \pm)$}

Obtained as white solid; yield: $17 \%$ (232 mg); mp: $182-184{ }^{\circ} \mathrm{C} ; R_{\mathrm{f}}$ 0.47 (6\% $\mathrm{MeOH}$ in $\left.\mathrm{CHCl}_{3}\right)$; IR $\left(\mathrm{KBr}, \nu_{\max } \mathrm{cm}^{-1}\right)$ : 3385, 2959, 2868, 1719, 1607; ${ }^{1} \mathrm{H}$ NMR $\left(P y-d_{5}\right): \delta 10.96(2 \mathrm{H}, \mathrm{s},-\mathrm{NH}), 7.32(1 \mathrm{H}$, $\mathrm{dd}, J=2.4,8.4 \mathrm{~Hz}), 7.27(2 \mathrm{H}, \mathrm{m}), 7.23(1 \mathrm{H}, \mathrm{d}, J=1.8 \mathrm{~Hz}), 7.22$ $(1 \mathrm{H}, \mathrm{m}), 7.16(1 \mathrm{H}, \mathrm{d}, J=1.8 \mathrm{~Hz}), 7.09(1 \mathrm{H}, \mathrm{dd}, J=1.8,7.8 \mathrm{~Hz})$, $6.96(2 \mathrm{H}, \mathrm{m}), 6.80(1 \mathrm{H}, \mathrm{m}), 6.77(1 \mathrm{H}, \mathrm{s}), 5.74(1 \mathrm{H}, \mathrm{s}), 4.52(1 \mathrm{H}, \mathrm{d}$, $J=12.6 \mathrm{~Hz}), 4.38(1 \mathrm{H}, \mathrm{d}, J=12.0 \mathrm{~Hz}), 4.33(1 \mathrm{H}, \mathrm{m}), 4.16(1 \mathrm{H}, \mathrm{m})$, $3.79(2 \mathrm{H}, \mathrm{m}), 3.75(3 \mathrm{H}, \mathrm{s}), 3.70(3 \mathrm{H}, \mathrm{s}), 2.80(1 \mathrm{H}, \mathrm{m}), 2.76(1 \mathrm{H}$, m), 2.62 (2H, m), $2.24(3 \mathrm{H}, \mathrm{s}), 2.20(3 \mathrm{H}, \mathrm{s}), 1.82(6 \mathrm{H}, \mathrm{m}), 1.69$ $(2 \mathrm{H}, \mathrm{m}) \cdot{ }^{13} \mathrm{C}$ NMR $\left(P y-d_{5}\right): \delta 192.3(\mathrm{C}=\mathrm{O}), 190.2(-\mathrm{C}=\mathrm{O}), 181.2$ $(\mathrm{C}=\mathrm{O}), 181.0(\mathrm{C}=\mathrm{O}), 158.7\left(\mathrm{C},{ }^{1} J_{\mathrm{C}-\mathrm{F}}=240.0 \mathrm{~Hz}\right), 149.21(\mathrm{C})$, 149.16 (C), 147.5 (C), 147.4 (C), 139.8 (C), 139.6 (C), $131.9(\mathrm{CH})$, 131.6 (C), 131.5 (C), 130.6 (C), $128.3\left(\mathrm{C},{ }^{3} J_{\mathrm{C}-\mathrm{F}}=7.5 \mathrm{~Hz}\right), 126.1$ (C), $125.4(\mathrm{CH}), 120.91(\mathrm{CH}), 120.87(\mathrm{CH}), 119.3(\mathrm{C}), 117.2(\mathrm{CH})$, $117.1(\mathrm{CH}), 116.1\left(\mathrm{CH},{ }^{2} J_{\mathrm{C}-\mathrm{F}}=22.5 \mathrm{~Hz}\right), 115.1\left(\mathrm{CH},{ }^{2} J_{\mathrm{C}-\mathrm{F}}=24.0\right.$ $\mathrm{Hz}), 112.4(\mathrm{CH}), 111.6(\mathrm{CH}), 111.1(\mathrm{CH}), 101.7$ (-CH), $74.9(\mathrm{C})$, $74.8(\mathrm{C}), 73.8(\mathrm{CH}), 73.3(\mathrm{CH}), 65.6(\mathrm{CH}), 63.8(\mathrm{CH}), 56.1(\mathrm{OMe})$, 56.0 (OMe), $52.4(\mathrm{CH}), 52.3(\mathrm{CH}), 48.5\left(\mathrm{CH}_{2}\right), 48.4\left(\mathrm{CH}_{2}\right), 31.1$ $\left(\mathrm{CH}_{2}\right), 30.7\left(\mathrm{CH}_{2}\right), 28.0\left(\mathrm{CH}_{2}\right), 27.7\left(\mathrm{CH}_{2}\right), 21.2\left(\mathrm{CH}_{3}\right), 17.1\left(\mathrm{CH}_{3}\right)$. MS [ESI-MS, positive mode]: found $m / z 815[\mathrm{M}+\mathrm{H}]^{+}, 837[\mathrm{M}+$ $\mathrm{Na}]^{+}$. HRMS [ESI-MS, positive mode]: $\mathrm{MF}: \mathrm{C}_{47} \mathrm{H}_{47} \mathrm{~N}_{4} \mathrm{O}_{8} \mathrm{~F}$; found $m / z$ 815.3458 [M $+\mathrm{H}]^{+}$[calcd. 815.3456].

\section{Spectral data of compound $4 \mathrm{Fb}( \pm)$}

Obtained as white solid; yield: $20 \%(273 \mathrm{mg})$; $\mathrm{mp}: 184-186^{\circ} \mathrm{C} ; R_{\mathrm{f}}$ 0.43 (6\% $\mathrm{MeOH}$ in $\mathrm{CHCl}_{3}$ ); IR ( $\left.\mathrm{KBr}, \nu_{\max } \mathrm{cm}^{-1}\right)$ : 3402, 2958, 2869, 1714, 1613, 1518; ${ }^{1} \mathrm{H}$ NMR $\left(P y-d_{5}\right): \delta 12.03(1 \mathrm{H}, \mathrm{s},-\mathrm{NH})$, $11.73(1 \mathrm{H}, \mathrm{s},-\mathrm{NH}), 7.28(1 \mathrm{H}, \mathrm{dd}, J=2.4,7.8 \mathrm{~Hz}), 7.19(1 \mathrm{H}, \mathrm{s})$, $7.16(1 \mathrm{H}, \mathrm{d}, J=8.4 \mathrm{~Hz}), 7.13(2 \mathrm{H}, \mathrm{m}), 7.08(1 \mathrm{H}, \mathrm{d}, J=1.8 \mathrm{~Hz})$, $6.98(1 \mathrm{H}, \mathrm{td}, J=2.4,9.0 \mathrm{~Hz}), 6.82(3 \mathrm{H}, \mathrm{m}), 6.69(1 \mathrm{H}, \mathrm{s}), 5.92(1 \mathrm{H}$, $\mathrm{s}), 4.38(2 \mathrm{H}, \mathrm{m}), 4.20(1 \mathrm{H}, \mathrm{m}), 4.13(1 \mathrm{H}, \mathrm{m}), 3.92(1 \mathrm{H}, \mathrm{m}), 3.78$ $(1 \mathrm{H}, \mathrm{m}), 3.67(3 \mathrm{H}, \mathrm{s}), 3.64(3 \mathrm{H}, \mathrm{s}), 2.85(1 \mathrm{H}, \mathrm{m}), 2.67(3 \mathrm{H}, \mathrm{m})$, $2.28(3 \mathrm{H}, \mathrm{s}), 2.12(3 \mathrm{H}, \mathrm{s}), 1.92(1 \mathrm{H}, \mathrm{m}), 1.85(2 \mathrm{H}, \mathrm{m}), 1.81(2 \mathrm{H}$, $\mathrm{m}), 1.71(3 \mathrm{H}, \mathrm{m}) .{ }^{13} \mathrm{C} \mathrm{NMR}\left(P y-d_{5}\right): \delta 190.6(\mathrm{C}=\mathrm{O}), 190.3(\mathrm{C}=\mathrm{O})$, $181.5(\mathrm{C}=\mathrm{O}), 181.3(\mathrm{C}=\mathrm{O}), 158.6\left(\mathrm{C},{ }^{1} J_{\mathrm{C}-\mathrm{F}}=237.0 \mathrm{~Hz}\right), 149.0(2$ $\times$ C), $147.44(\mathrm{C}), 147.38(\mathrm{C}), 139.9(2 \times \mathrm{C}), 131.9(\mathrm{CH}), 131.6(\mathrm{C})$, 131.3 (C), 130.6 (C), $128.4\left(\mathrm{C},{ }^{3} J_{\mathrm{C}-\mathrm{F}}=7.5 \mathrm{~Hz}\right), 126.2$ (C), 125.5 $(\mathrm{CH}), 120.3(\mathrm{CH}), 120.2(\mathrm{CH}), 119.4(\mathrm{C}), 117.0(\mathrm{CH}), 116.9(\mathrm{CH})$, $116.2\left(\mathrm{CH},{ }^{2} J_{\mathrm{C}-\mathrm{F}}=24.0 \mathrm{~Hz}\right), 115.1\left(\mathrm{CH},{ }^{2} J_{\mathrm{C}-\mathrm{F}}=24.0 \mathrm{~Hz}\right), 112.6$ $(\mathrm{CH}), 112.3(\mathrm{CH}), 111.0\left(\mathrm{CH},{ }^{3} J_{\mathrm{C}-\mathrm{F}}=7.5 \mathrm{~Hz}\right), 101.4(\mathrm{CH}), 75.44$ (C), $75.41(\mathrm{C}), 73.6(\mathrm{CH}), 73.4(\mathrm{CH}), 65.3(\mathrm{CH}), 64.9(\mathrm{CH}), 56.03$ (OMe), 55.98 (OMe), $51.8(\mathrm{CH}), 51.5(\mathrm{CH}), 48.33\left(\mathrm{CH}_{2}\right), 48.29$ $\left(\mathrm{CH}_{2}\right), 31.5\left(\mathrm{CH}_{2}\right), 31.3\left(\mathrm{CH}_{2}\right), 28.4\left(\mathrm{CH}_{2}\right), 28.2\left(\mathrm{CH}_{2}\right), 21.3\left(\mathrm{CH}_{3}\right)$, $17.2\left(\mathrm{CH}_{3}\right)$. MS [ESI-MS, positive mode]: found $m / z 815[\mathrm{M}+\mathrm{H}]^{+}$, 
$837[\mathrm{M}+\mathrm{Na}]^{+}$. HRMS [ESI-MS, positive mode]: MF: $\mathrm{C}_{47} \mathrm{H}_{47} \mathrm{~N}_{4} \mathrm{O}_{8} \mathrm{~F}$; found $\mathrm{m} / z$ 815.3456 [M $\left.+\mathrm{H}\right]^{+}$[calcd. 815.3456].

\section{Spectral data of compound $4 \mathrm{Fc}( \pm)$}

Obtained as white solid; yield: $20 \%(273 \mathrm{mg}) ; \mathrm{mp}: 173-175^{\circ} \mathrm{C} ; R_{\mathrm{f}}$ $0.37\left(6 \% \mathrm{MeOH}\right.$ in $\left.\mathrm{CHCl}_{3}\right) ;{ }^{1} \mathrm{H}$ NMR $\left(P y-d_{5}\right): \delta 11.77(1 \mathrm{H}, \mathrm{s},-\mathrm{NH})$, $11.38(1 \mathrm{H}, \mathrm{s},-\mathrm{NH}), 7.63(1 \mathrm{H}, \mathrm{m}), 7.44(1 \mathrm{H}, \mathrm{s}), 7.34(1 \mathrm{H}, \mathrm{s}), 7.16$ $(1 \mathrm{H}, \mathrm{d}, J=8.4 \mathrm{~Hz}), 7.12(1 \mathrm{H}, \mathrm{m}), 7.00(1 \mathrm{H}, \mathrm{td}, J=2.4,9.0 \mathrm{~Hz})$, $6.91(2 \mathrm{H}, \mathrm{m}), 6.86(2 \mathrm{H}, \mathrm{m}), 6.64(1 \mathrm{H}, \mathrm{q}, J=4.2 \mathrm{~Hz}), 6.10(1 \mathrm{H}, \mathrm{s})$, $4.99(1 \mathrm{H}, \mathrm{m}), 4.74(1 \mathrm{H}, \mathrm{m}), 4.65(1 \mathrm{H}, \mathrm{d}, J=12.0 \mathrm{~Hz}), 4.33$ $(1 \mathrm{H}, \mathrm{m}), 4.14(1 \mathrm{H}, \mathrm{d}, J=12.0 \mathrm{~Hz}), 4.05(1 \mathrm{H}, \mathrm{m}), 3.67(3 \mathrm{H}, \mathrm{s}), 3.51$ $(3 \mathrm{H}, \mathrm{s}), 3.22(1 \mathrm{H}, \mathrm{m}), 2.95(1 \mathrm{H}, \mathrm{m}), 2.83(1 \mathrm{H}, \mathrm{m}), 2.75(1 \mathrm{H}, \mathrm{m})$, $2.39(3 \mathrm{H}, \mathrm{s}), 2.28(3 \mathrm{H}, \mathrm{s}), 1.95(3 \mathrm{H}, \mathrm{m}), 1.78(3 \mathrm{H}, \mathrm{m}), 1.69(2 \mathrm{H}$, m). ${ }^{13} \mathrm{C}$ NMR $\left(P y-d_{5}\right): \delta 195.7(\mathrm{C}=\mathrm{O}), 189.0(\mathrm{C}=\mathrm{O}), 181.6(\mathrm{C}=\mathrm{O})$, $180.1(\mathrm{C}=\mathrm{O}), 158.9\left(\mathrm{C},{ }^{1} J_{\mathrm{C}-\mathrm{F}}=235.5 \mathrm{~Hz}\right), 149.2(\mathrm{C}), 148.3$ (C), 147.61 (C), 147.58 (C), 140.9 (C), 140.3 (C), 131.9 (CH), 131.6 (C), $130.8(\mathrm{C}), 129.8\left(\mathrm{C},{ }^{3} J_{\mathrm{C}-\mathrm{F}}=7.4 \mathrm{~Hz}\right), 126.8(\mathrm{C}), 126.7(\mathrm{C}), 125.7$ (CH), $121.7(\mathrm{CH}), 121.2(\mathrm{CH}), 119.5(\mathrm{C}), 117.1(\mathrm{CH}), 116.4(\mathrm{CH})$, $116.1\left(\mathrm{CH},{ }^{2} J_{\mathrm{C}-\mathrm{F}}=22.5 \mathrm{~Hz}\right), 115.2\left(\mathrm{CH},{ }^{2} J_{\mathrm{C}-\mathrm{F}}=24.0 \mathrm{~Hz}\right), 112.7$ $(\mathrm{CH}), 112.4(\mathrm{CH}), 110.9\left(\mathrm{CH},{ }^{3} J_{\mathrm{C}-\mathrm{F}}=7.5 \mathrm{~Hz}\right), 102.8(\mathrm{CH}), 76.8(\mathrm{C})$, $75.2(\mathrm{C}), 73.8(\mathrm{CH}), 65.9(\mathrm{CH}), 64.6(\mathrm{CH}), 56.1(\mathrm{OMe}), 55.7(\mathrm{OMe})$, $53.2(\mathrm{CH}), 52.6(\mathrm{CH}), 52.0(\mathrm{CH}), 51.5\left(\mathrm{CH}_{2}\right), 48.6\left(\mathrm{CH}_{2}\right), 31.3$ $\left(\mathrm{CH}_{2}\right), 29.0\left(\mathrm{CH}_{2}\right), 28.2\left(\mathrm{CH}_{2}\right), 26.9\left(\mathrm{CH}_{2}\right), 21.3\left(\mathrm{CH}_{3}\right), 17.4\left(\mathrm{CH}_{3}\right)$. MS [ESI-MS, positive mode]: found $m / z 815[\mathrm{M}+\mathrm{H}]^{+}, 837[\mathrm{M}+$ $\mathrm{Na}]^{+}$. HRMS [ESI-MS, positive mode]: MF: $\mathrm{C}_{47} \mathrm{H}_{47} \mathrm{~N}_{4} \mathrm{O}_{8} \mathrm{~F}$; found $\mathrm{m} / \mathrm{z}$ 815.3462 [M + H] $]^{+}$[calcd. 815.3456].

\section{Spectral data of compound $4 \mathrm{Fd}( \pm)$}

Obtained as white solid; yield: $22 \%(300 \mathrm{mg}) ; \mathrm{mp}: 186-188^{\circ} \mathrm{C} ; R_{\mathrm{f}}$ $0.36\left(6 \% \mathrm{MeOH}\right.$ in $\left.\mathrm{CHCl}_{3}\right) ;{ }^{1} \mathrm{H}$ NMR $\left(P y-d_{5}\right): \delta 11.73(1 \mathrm{H}, \mathrm{s},-\mathrm{NH})$, $11.37(1 \mathrm{H}, \mathrm{s},-\mathrm{NH}), 7.66(1 \mathrm{H}, \mathrm{d}, J=7.8 \mathrm{~Hz}), 7.54(1 \mathrm{H}, \mathrm{s}), 7.41(1 \mathrm{H}$, $\mathrm{s}), 7.18(2 \mathrm{H}, \mathrm{s}), 7.01(1 \mathrm{H}, \mathrm{td}, J=3.0,9.0 \mathrm{~Hz}), 6.95(1 \mathrm{H}, \mathrm{dd}, J=1.8$, $8.4 \mathrm{~Hz}), 6.90(3 \mathrm{H}, \mathrm{m}), 6.68(1 \mathrm{H}, \mathrm{q}, J=4.2 \mathrm{~Hz}), 6.00(1 \mathrm{H}, \mathrm{s}), 4.91$ $(1 \mathrm{H}, \mathrm{m}), 4.71(1 \mathrm{H}, \mathrm{m}), 4.64(1 \mathrm{H}, \mathrm{d}, J=12.0 \mathrm{~Hz}), 4.40(1 \mathrm{H}, \mathrm{m})$, $4.29(1 \mathrm{H}, \mathrm{d}, J=12.0 \mathrm{~Hz}), 4.12(1 \mathrm{H}, \mathrm{m}), 3.70(3 \mathrm{H}, \mathrm{s}), 3.49(3 \mathrm{H}, \mathrm{s})$, $3.18(1 \mathrm{H}, \mathrm{m}), 2.97(1 \mathrm{H}, \mathrm{m}), 2.76(2 \mathrm{H}, \mathrm{m}), 2.37(3 \mathrm{H}, \mathrm{s}), 2.34(3 \mathrm{H}$, s), $2.25(1 \mathrm{H}, \mathrm{m}), 1.96(3 \mathrm{H}, \mathrm{m}), 1.79(1 \mathrm{H}, \mathrm{m}), 1.73(1 \mathrm{H}, \mathrm{m}), 1.63$ (1H, m), $1.58(1 \mathrm{H}, \mathrm{m}) .{ }^{13} \mathrm{C}$ NMR $\left(P y-d_{5}\right): \delta 195.2(\mathrm{C}=\mathrm{O}), 190.8$ $(\mathrm{C}=\mathrm{O}), 185.8(\mathrm{C}=\mathrm{O}), 180.3(\mathrm{C}=\mathrm{O}), 158.9\left(\mathrm{C},{ }^{1} J_{\mathrm{C}-\mathrm{F}}=235.5 \mathrm{~Hz}\right)$, 149.2 (C), 148.2 (C), 147.45 (C), 147.37 (C), 140.9 (C), 140.2 (C), 131.9 (CH), 130.7 (C), 130.2 (C), 129.6 (C), 127.75 (C), 127.0 (C), $126.1(\mathrm{CH}), 121.6(\mathrm{CH}), 121.4(\mathrm{CH}), 119.5(\mathrm{C}), 117.0(\mathrm{CH})$, $116.3(\mathrm{CH}), 116.0\left(\mathrm{CH},{ }^{2} J_{\mathrm{C}-\mathrm{F}}=24.0 \mathrm{~Hz}\right), 115.9\left(\mathrm{CH},{ }^{2} J_{\mathrm{C}-\mathrm{F}}=25.5\right.$ $\mathrm{Hz}), 113.3(\mathrm{CH}), 112.5(\mathrm{CH}), 110.8\left(\mathrm{CH},{ }^{3} J_{\mathrm{C}-\mathrm{F}}=7.5 \mathrm{~Hz}\right), 102.85$ $(\mathrm{CH}), 76.8(\mathrm{C}), 75.7(\mathrm{C}), 73.7(\mathrm{CH}), 65.1(\mathrm{CH}), 64.7(\mathrm{CH}), 56.1$ (OMe), $55.7(\mathrm{OMe}), 54.4(\mathrm{CH}), 52.6(\mathrm{CH}), 51.3\left(\mathrm{CH}, \mathrm{CH}_{2}\right), 48.7$ $\left(\mathrm{CH}_{2}\right), 31.3\left(\mathrm{CH}_{2}\right), 29.2\left(\mathrm{CH}_{2}\right), 28.2\left(\mathrm{CH}_{2}\right), 26.8\left(\mathrm{CH}_{2}\right), 21.4\left(\mathrm{CH}_{3}\right)$, 17.4 $\left(\mathrm{CH}_{3}\right)$. MS [ESI-MS, positive mode]: found $\mathrm{m} / z 815[\mathrm{M}+\mathrm{H}]^{+}$, $837[\mathrm{M}+\mathrm{Na}]^{+}$. HRMS [ESI-MS, positive mode]: MF: $\mathrm{C}_{47} \mathrm{H}_{47} \mathrm{~N}_{4} \mathrm{O}_{8} \mathrm{~F}$; found $m / z$ 815.3452 [M + H] $]^{+}$[calcd. 815.3456].

\section{Spectral data of compound 5Aa $( \pm)$}

Obtained as white solid; yield: $30 \%(655 \mathrm{mg}) ; \mathrm{mp}: 230-232{ }^{\circ} \mathrm{C} ; R_{\mathrm{f}}$ $0.65\left(5 \% \mathrm{MeOH}\right.$ in $\left.\mathrm{CHCl}_{3}\right)$; UV absorption maximas $\left(\lambda_{\max } \mathrm{nm}\right.$, methanol) 297, 229; IR (KBr, $\left.\nu_{\max } \mathrm{cm}^{-1}\right): 3336,2927,1725,1615$, 1517; ${ }^{1} \mathrm{H}$ NMR $\left(P y-d_{5}\right): \delta 11.26(2 \mathrm{H}, \mathrm{s},-\mathrm{NH}), 7.64(2 \mathrm{H}, \mathrm{d}, J=$
$7.8 \mathrm{~Hz}), 7.31(2 \mathrm{H}, \mathrm{m}), 7.25(2 \mathrm{H}, \mathrm{dd}, J=0.6,7.8 \mathrm{~Hz}), 7.22(2 \mathrm{H}, \mathrm{m})$, $6.99(4 \mathrm{H}, \mathrm{m}), 6.93(2 \mathrm{H}, \mathrm{d}, J=7.8 \mathrm{~Hz}), 5.74(1 \mathrm{H}, \mathrm{s}), 4.36(2 \mathrm{H}, \mathrm{d}, J=$ $12.6 \mathrm{~Hz}), 4.33(2 \mathrm{H}, \mathrm{m}), 3.92(2 \mathrm{H}, \mathrm{d}, J=10.2 \mathrm{~Hz}), 3.76(6 \mathrm{H}, \mathrm{s}), 3.65$ $(4 \mathrm{H}, \mathrm{m}), 3.11(2 \mathrm{H}, \mathrm{dd}, J=2.4,11.4 \mathrm{~Hz}), 3.00(2 \mathrm{H}, \mathrm{m}) .{ }^{13} \mathrm{C}$ NMR $\left(P y-d_{5}\right): \delta 190.5(\mathrm{C}=\mathrm{O}), 180.0(\mathrm{C}=\mathrm{O}), 149.5(\mathrm{C}), 148.0(\mathrm{C}), 143.8$ (C), $130.5(\mathrm{C}), 130.3(\mathrm{CH}), 128.5(\mathrm{CH}), 124.5(\mathrm{C}), 121.7(\mathrm{CH})$, $121.5(\mathrm{CH}), 117.3(\mathrm{CH}), 111.7(\mathrm{CH}), 110.5(\mathrm{CH}), 101.4(\mathrm{CH}), 76.7$ (CH), $75.1(\mathrm{C}), 62.6(\mathrm{CH}), 56.2(\mathrm{OMe}), 54.9\left(\mathrm{CH}_{2}\right), 51.5(\mathrm{CH}), 36.7$ $\left(\mathrm{CH}_{2}\right)$. MS [ESI-MS, positive mode]: found $m / z 827[\mathrm{M}+\mathrm{Na}]^{+}$. HRMS [ESI-MS, positive mode]: MF: $\mathrm{C}_{43} \mathrm{H}_{40} \mathrm{~N}_{4} \mathrm{O}_{8} \mathrm{~S}_{2}$; found $\mathrm{m} / \mathrm{z}$ $827.2180[\mathrm{M}+\mathrm{Na}]^{+}$[calcd. 827.2185].

\section{Spectral data of compound $5 \mathrm{Ab}( \pm)$}

Obtained as white solid; yield: $35 \%(765 \mathrm{mg}) ; \mathrm{mp}: 242-244^{\circ} \mathrm{C} ; R_{\mathrm{f}}$ 0.55 ( $5 \% \mathrm{MeOH}$ in $\left.\mathrm{CHCl}_{3}\right)$; UV absorption maximas $\left(\lambda_{\max } \mathrm{nm}\right.$, methanol) 304, 228; IR ( $\left.\mathrm{KBr}, \nu_{\max } \mathrm{cm}^{-1}\right): 3407,2928,1718,1617$, 1518; ${ }^{1} \mathrm{H}$ NMR $\left(P y-d_{5}\right): \delta 11.94(2 \mathrm{H}, \mathrm{s},-\mathrm{NH}), 7.43(4 \mathrm{H}, \mathrm{m}), 7.30$ $(2 \mathrm{H}, \mathrm{d}, J=1.8 \mathrm{~Hz}), 7.20(1 \mathrm{H}, \mathrm{d}, J=1.8 \mathrm{~Hz}), 7.18(3 \mathrm{H}, \mathrm{t}, J=7.8$ $\mathrm{Hz}), 6.94(4 \mathrm{H}, \mathrm{m}), 5.98(1 \mathrm{H}, \mathrm{s}), 4.36(2 \mathrm{H}, \mathrm{d}, J=13.2 \mathrm{~Hz}), 4.32$ $(2 \mathrm{H}, \mathrm{t}, J=7.8 \mathrm{~Hz}), 3.92(2 \mathrm{H}, \mathrm{d}, J=10.8 \mathrm{~Hz}), 3.86(6 \mathrm{H}, \mathrm{s}), 3.63$ $(4 \mathrm{H}, \mathrm{m}), 3.15(2 \mathrm{H}, \mathrm{d}, J=11.4 \mathrm{~Hz}), 3.00(2 \mathrm{H}, \mathrm{dd}, J=6.6,11.4 \mathrm{~Hz})$. ${ }^{13} \mathrm{C}$ NMR $\left(P y-d_{5}\right): \delta 190.5(\mathrm{C}=\mathrm{O}), 180.3(\mathrm{C}=\mathrm{O}), 148.2(2 \times \mathrm{C})$, $144.0(\mathrm{C}), 130.3(\mathrm{CH}), 130.2(\mathrm{C}), 128.4(\mathrm{CH}), 123.5(\mathrm{C}), 121.8$ $(\mathrm{CH}), 121.6(\mathrm{CH}), 117.5(\mathrm{CH}), 111.7(\mathrm{CH}), 110.5(\mathrm{CH}), 101.1(\mathrm{CH})$, $76.9(\mathrm{CH}), 75.5(\mathrm{C}), 61.6(\mathrm{CH}), 56.3(\mathrm{OMe}), 55.9\left(\mathrm{CH}_{2}\right), 51.9(\mathrm{CH})$, $36.9\left(\mathrm{CH}_{2}\right)$. MS [ESI-MS, positive mode]: found $\mathrm{m} / \mathrm{z} 827[\mathrm{M}+$ $\mathrm{Na}]^{+}$. HRMS [ESI-MS, positive mode]: MF: $\mathrm{C}_{43} \mathrm{H}_{40} \mathrm{~N}_{4} \mathrm{O}_{8} \mathrm{~S}_{2}$; found $\mathrm{m} / \mathrm{z}$ 827.2167 [M + Na $]^{+}$[calcd. 827.2185].

\section{Spectral data of compound 5Ba $( \pm)$}

Obtained as white solid; yield: $31 \%$ (700 mg); mp: $216-218^{\circ} \mathrm{C} ; R_{\mathrm{f}}$ 0.60 (5\% $\mathrm{MeOH}$ in $\left.\mathrm{CHCl}_{3}\right)$; IR $\left(\mathrm{KBr}, \nu_{\max } \mathrm{cm}^{-1}\right): 3379,2923$, $1718,1602,1521 ;{ }^{1} \mathrm{H}$ NMR $\left(P y-d_{5}\right): \delta 10.96(2 \mathrm{H}, \mathrm{s},-\mathrm{NH}), 7.52(2 \mathrm{H}$, s), $7.27(2 \mathrm{H}, \mathrm{d}, J=8.4 \mathrm{~Hz}), 7.22(2 \mathrm{H}, \mathrm{m}), 7.00(2 \mathrm{H}, \mathrm{dd}, J=1.8,8.4$ $\mathrm{Hz}), 6.94(2 \mathrm{H}, \mathrm{d}, J=8.4 \mathrm{~Hz}), 6.84(2 \mathrm{H}, \mathrm{d}, J=7.8 \mathrm{~Hz}), 5.66(1 \mathrm{H}, \mathrm{s})$, $4.36(2 \mathrm{H}, \mathrm{m}), 4.30(2 \mathrm{H}, \mathrm{d}, J=12.0 \mathrm{~Hz}), 3.88(2 \mathrm{H}, \mathrm{d}, J=10.2 \mathrm{~Hz})$, $3.78(2 \mathrm{H}, \mathrm{m}), 3.73(6 \mathrm{H}, \mathrm{s}), 3.60(2 \mathrm{H}, \mathrm{d}, J=9.6 \mathrm{~Hz}), 3.10(2 \mathrm{H}, \mathrm{dd}$, $J=2.4,10.8 \mathrm{~Hz}), 2.98(2 \mathrm{H}, \mathrm{m}), 2.12(6 \mathrm{H}, \mathrm{s}) .{ }^{13} \mathrm{C} \mathrm{NMR}\left(P y-d_{5}\right)$ : $\delta 190.2(\mathrm{C}=\mathrm{O}), 180.2(\mathrm{C}=\mathrm{O}), 149.3(\mathrm{C}), 147.7(\mathrm{C}), 141.1(\mathrm{C})$, $130.9(\mathrm{C}), 130.724(\mathrm{C}), 130.716(\mathrm{CH}), 129.1(\mathrm{CH}), 124.6(\mathrm{C}), 121.2$ (CH), 117.3 (CH), 112.2 (CH), 110.3 (CH), 101.6 (CH), $76.4(\mathrm{CH})$, $75.2(\mathrm{C}), 62.9(\mathrm{CH}), 56.2(\mathrm{OMe}), 54.6\left(\mathrm{CH}_{2}\right), 50.9(\mathrm{CH}), 36.7$ $\left(\mathrm{CH}_{2}\right), 21.3\left(\mathrm{CH}_{3}\right)$. MS [ESI-MS, positive mode]: found $\mathrm{m} / \mathrm{z} 855$ $[\mathrm{M}+\mathrm{Na}]^{+}$. HRMS [ESI-MS, positive mode]: MF: $\mathrm{C}_{45} \mathrm{H}_{44} \mathrm{~N}_{4} \mathrm{O}_{8} \mathrm{~S}_{2}$; found $m / z$ 855.2495 [M + Na] ${ }^{+}$[calcd. 855.2498].

\section{Spectral data of compound $\mathbf{5 B b}( \pm)$}

Obtained as white solid; yield: $35 \%$ (791 mg); mp: $260-262{ }^{\circ} \mathrm{C} ; R_{\mathrm{f}}$ $0.53\left(5 \% \mathrm{MeOH}\right.$ in $\left.\mathrm{CHCl}_{3}\right)$; IR ( $\left.\mathrm{KBr}, \nu_{\max } \mathrm{cm}^{-1}\right): 3388,2921$, $1708,1617,1516 ;{ }^{1} \mathrm{H}$ NMR $\left(P y-d_{5}\right): \delta 11.93(2 \mathrm{H}, \mathrm{s},-\mathrm{NH}), 7.55(2 \mathrm{H}$, s), $7.17(2 \mathrm{H}, \mathrm{d}, J=7.8 \mathrm{~Hz}), 7.11(2 \mathrm{H}, \mathrm{d}, J=1.8 \mathrm{~Hz}), 6.93(2 \mathrm{H}, \mathrm{d}$, $J=7.8 \mathrm{~Hz}), 6.88(2 \mathrm{H}, \mathrm{dd}, J=1.2,7.8 \mathrm{~Hz}), 6.79(2 \mathrm{H}, \mathrm{d}, J=7.8 \mathrm{~Hz})$, $5.88(1 \mathrm{H}, \mathrm{s}), 4.30(2 \mathrm{H}, \mathrm{m}), 4.27(2 \mathrm{H}, \mathrm{d}, J=12.0 \mathrm{~Hz}), 3.95(2 \mathrm{H}, \mathrm{d}$, $J=10.2 \mathrm{~Hz}), 3.86(2 \mathrm{H}, \mathrm{m}), 3.70(2 \mathrm{H}, \mathrm{d}, J=10.2 \mathrm{~Hz}), 3.67(6 \mathrm{H}, \mathrm{s})$, $3.15(2 \mathrm{H}, \mathrm{dd}, J=1.8,11.4 \mathrm{~Hz}), 3.02(2 \mathrm{H}, \mathrm{m}), 2.08(6 \mathrm{H}, \mathrm{s}) .{ }^{13} \mathrm{C}$ NMR $\left(P y-d_{5}\right): \delta 189.9(\mathrm{C}=\mathrm{O}), 180.3(\mathrm{C}=\mathrm{O}), 149.2(\mathrm{C}), 147.8(\mathrm{C})$, 
$141.4(\mathrm{C}), 130.8(\mathrm{CH}), 130.7$ (C), 130.5 (C), $129.2(\mathrm{CH}), 124.6$ (C), $120.8(\mathrm{CH}), 117.2(\mathrm{CH}), 112.4(\mathrm{CH}), 110.0(\mathrm{CH}), 101.3(\mathrm{CH}), 76.7$ $(\mathrm{CH}), 75.9(\mathrm{C}), 63.0(\mathrm{CH}), 56.0(\mathrm{OMe}), 55.1\left(\mathrm{CH}_{2}\right), 50.8(\mathrm{CH}), 37.0$ $\left(\mathrm{CH}_{2}\right), 21.3\left(\mathrm{CH}_{3}\right)$. MS [ESI-MS, positive mode]: found $\mathrm{m} / \mathrm{z} 855$ [M $+\mathrm{Na}]^{+}$. HRMS [ESI-MS, positive mode]: MF: $\mathrm{C}_{45} \mathrm{H}_{44} \mathrm{~N}_{4} \mathrm{O}_{8} \mathrm{~S}_{2}$; found $\mathrm{m} / \mathrm{z}$ 855.2498 [M+ Na] $]^{+}$[calcd. 855.2498].

\section{Spectral data of compound 6Aa $( \pm)$}

Color: Obtained as white solid; Yield: 30\% (431 mg); Mp: 172$174{ }^{\circ} \mathrm{C} ; R_{\mathrm{f}} 0.60$ (5\% MeOH in $\mathrm{CHCl}_{3}$ ); UV absorption maximas ( $\lambda_{\max } \mathrm{nm}$, methanol) 258, 207; IR ( $\left.\mathrm{KBr}, \nu_{\max } \mathrm{cm}^{-1}\right): 3290,2926$, 1720, 1608, 1520; ${ }^{1} \mathrm{H}$ NMR $\left(P y-d_{5}\right): \delta 11.13(2 \mathrm{H}, \mathrm{s},-\mathrm{NH}), 7.61(1 \mathrm{H}$, s), $7.32(2 \mathrm{H}, \mathrm{d}, J=8.4 \mathrm{~Hz}), 7.26(1 \mathrm{H}, \mathrm{d}, J=8.4 \mathrm{~Hz}), 7.18(2 \mathrm{H}, \mathrm{m})$, $7.15(1 \mathrm{H}, \mathrm{m}), 7.13(1 \mathrm{H}, \mathrm{s}), 6.94(4 \mathrm{H}, \mathrm{m}), 6.81(1 \mathrm{H}, \mathrm{m}), 5.77(1 \mathrm{H}, \mathrm{s})$, $4.49(1 \mathrm{H}, \mathrm{d}, J=12.0 \mathrm{~Hz}), 4.36(1 \mathrm{H}, \mathrm{d}, J=12.6 \mathrm{~Hz}), 4.32(1 \mathrm{H}, \mathrm{m})$, $4.23(1 \mathrm{H}, \mathrm{m}), 3.96(1 \mathrm{H}, \mathrm{d}, J=9.6 \mathrm{~Hz}), 3.81(3 \mathrm{H}, \mathrm{s}), 3.73(3 \mathrm{H}, \mathrm{s}), 3.70$ $(1 \mathrm{H}, \mathrm{m}), 3.68(3 \mathrm{H}, \mathrm{s}), 3.63(2 \mathrm{H}, \mathrm{m}), 3.08(1 \mathrm{H}, \mathrm{d}, J=10.2 \mathrm{~Hz}), 2.98$ (1H, m), $2.76(1 \mathrm{H}, \mathrm{m}), 2.61(1 \mathrm{H}, \mathrm{m}), 1.89(1 \mathrm{H}, \mathrm{m}), 1.79(2 \mathrm{H}, \mathrm{m}), 1.67$ (1H, m). ${ }^{13} \mathrm{C}$ NMR $\left(P y-d_{5}\right): \delta 191.8(\mathrm{C}=\mathrm{O}), 189.9(\mathrm{C}=\mathrm{O}), 181.0(\mathrm{C}=$ O), 179.9 (C=O), 155.2 (C), 149.4 (C), 149.3 (C), 147.9 (C), 147.6 (C), 143.8 (C), 137.2 (C), 131.8 (C), 130.2 (C), 129.7 (CH), 126.94 (C), $126.89(\mathrm{CH}), 125.9(\mathrm{C}), 121.8(\mathrm{CH}), 121.6(\mathrm{CH}), 121.1(\mathrm{CH}), 117.3$ $(\mathrm{CH}), 117.2(\mathrm{CH}), 116.4(\mathrm{CH}), 115.0(\mathrm{CH}), 111.8(\mathrm{CH}), 111.5(\mathrm{CH})$, $110.8(\mathrm{CH}), 110.6(\mathrm{CH}), 101.6(\mathrm{CH}), 76.5(\mathrm{CH}), 75.7(\mathrm{C}), 74.4(\mathrm{C})$, $73.9(\mathrm{CH}), 64.0(\mathrm{CH}), 63.0(\mathrm{CH}), 56.19(\mathrm{OMe}), 56.16(\mathrm{OMe}), 56.12$ (OMe), $55.1\left(\mathrm{CH}_{2}\right), 52.7(\mathrm{CH}), 51.6(\mathrm{CH}), 48.6\left(\mathrm{CH}_{2}\right), 36.7\left(\mathrm{CH}_{2}\right)$, $30.9\left(\mathrm{CH}_{2}\right), 27.6\left(\mathrm{CH}_{2}\right)$. MS [ESI-MS, positive mode]: found $\mathrm{m} / \mathrm{z} 817$ $[\mathrm{M}+\mathrm{H}]^{+}, 839[\mathrm{M}+\mathrm{Na}]^{+}$. HRMS [ESI-MS, positive mode]: MF: $\mathrm{C}_{45} \mathrm{H}_{44} \mathrm{~N}_{4} \mathrm{O}_{9} \mathrm{~S}$; found $m / z$ 839.2722 [M $\left.+\mathrm{Na}\right]^{+}$[calcd. 839.2727].

\section{Spectral data of compound 6Ab $( \pm)$}

Obtained as white solid; yield: $35 \%$ (503 mg); mp: $176-178^{\circ} \mathrm{C} ; R_{\mathrm{f}}$ 0.55 (5\% $\mathrm{MeOH}$ in $\left.\mathrm{CHCl}_{3}\right)$; UV absorption maximas $\left(\lambda_{\max } \mathrm{nm}\right.$, methanol) 256, 201; ${ }^{1} \mathrm{H}$ NMR $\left(P y-d_{5}\right): \delta 11.91(1 \mathrm{H}, \mathrm{s},-\mathrm{NH}), 11.78$ $(1 \mathrm{H}, \mathrm{s},-\mathrm{NH}), 7.49(1 \mathrm{H}, \mathrm{d}, J=2.4 \mathrm{~Hz}), 7.22(2 \mathrm{H}, \mathrm{m}), 7.19(1 \mathrm{H}, \mathrm{m})$, $7.16(2 \mathrm{H}, \mathrm{dd}, J=1.8,7.8 \mathrm{~Hz}), 7.13(1 \mathrm{H}, \mathrm{d}, J=7.2 \mathrm{~Hz}), 7.01(1 \mathrm{H}$, $\mathrm{dd}, J=1.8,7.8 \mathrm{~Hz}), 6.94(4 \mathrm{H}, \mathrm{m}), 6.87(1 \mathrm{H}, \mathrm{dd}, J=2.4,8.4 \mathrm{~Hz})$, $5.95(1 \mathrm{H}, \mathrm{s}), 4.38(2 \mathrm{H}, \mathrm{m}), 4.28(1 \mathrm{H}, \mathrm{m}), 4.16(1 \mathrm{H}, \mathrm{m}), 3.97(1 \mathrm{H}, \mathrm{d}$, $J=10.8 \mathrm{~Hz}), 3.79(1 \mathrm{H}, \mathrm{m}), 3.76(3 \mathrm{H}, \mathrm{s}), 3.72(3 \mathrm{H}, \mathrm{s}), 3.68(1 \mathrm{H}, \mathrm{m})$, $3.63(3 \mathrm{H}, \mathrm{s}, 1 \mathrm{H}, \mathrm{m}), 3.13(1 \mathrm{H}, \mathrm{dd}, J=1.8,11.4 \mathrm{~Hz}), 3.00(1 \mathrm{H}, \mathrm{m})$, 2.69 (1H, m), 2.60 (1H, m), $1.85(1 \mathrm{H}, \mathrm{m}), 1.68(3 \mathrm{H}, \mathrm{m}) .{ }^{13} \mathrm{C} \mathrm{NMR}$ $\left(P y-d_{5}\right): \delta 191.2(\mathrm{C}=\mathrm{O}), 189.6(\mathrm{C}=\mathrm{O}), 181.1(\mathrm{C}=\mathrm{O}), 180.3(\mathrm{C}=$ O), 155.1 (C), 149.3 (C), 149.2 (C), 147.9 (C), 147.6 (C), 143.8 (C), 137.3 (C), 131.3 (C), 130.6 (C), 129.7 (CH), 127.1 (CH), 126.6 (C), $125.8(\mathrm{C}), 121.7(\mathrm{CH}), 121.3(\mathrm{CH}), 120.9(\mathrm{CH}), 117.2(\mathrm{CH}), 117.1$ $(\mathrm{CH}), 115.8(\mathrm{CH}), 115.3(\mathrm{CH}), 112.1(\mathrm{CH}), 111.9(\mathrm{CH}), 110.7(\mathrm{CH})$, 110.6 (CH), 101.2 (CH), 76.7 (CH), $76.1(\mathrm{C}), 74.9(\mathrm{C}), 73.8(\mathrm{CH})$, $64.1(\mathrm{CH}), 62.7$ (CH), 56.15 (OMe), 56.11 (OMe), 55.90 (OMe), $55.6\left(\mathrm{CH}_{2}\right), 52.3(\mathrm{CH}), 51.2(\mathrm{CH}), 48.5\left(\mathrm{CH}_{2}\right), 37.0\left(\mathrm{CH}_{2}\right), 31.3$ $\left(\mathrm{CH}_{2}\right), 28.0\left(\mathrm{CH}_{2}\right)$. MS [ESI-MS, positive mode]: found $\mathrm{m} / \mathrm{z} 817$ $[\mathrm{M}+\mathrm{H}]^{+}, 839[\mathrm{M}+\mathrm{Na}]^{+}$. HRMS [ESI-MS, positive mode]: MF: $\mathrm{C}_{45} \mathrm{H}_{44} \mathrm{~N}_{4} \mathrm{O}_{9} \mathrm{~S}$; found $\mathrm{m} / z$ 839.2728 [M + Na] $]^{+}$[calcd. 839.2727].

\section{Spectral data of compound $6 \mathrm{Ba}( \pm)$}

Obtained as white solid; yield: $32 \%$ (508 mg); mp: $212-214{ }^{\circ} \mathrm{C} ; R_{\mathrm{f}}$ 0.56 (5\% $\mathrm{MeOH}$ in $\left.\mathrm{CHCl}_{3}\right)$; IR $\left(\mathrm{KBr}, \nu_{\max } \mathrm{cm}^{-1}\right)$ : 3511, 3209,
2924, 1713, 1606, 1515; ${ }^{1} \mathrm{H}$ NMR $\left(P y-d_{5}\right): \delta 11.73(1 \mathrm{H}, \mathrm{s},-\mathrm{NH})$, $11.29(1 \mathrm{H}, \mathrm{s},-\mathrm{NH}), 8.38(1 \mathrm{H}, \mathrm{d}, J=1.2 \mathrm{~Hz}), 7.84(1 \mathrm{H}, \mathrm{d}, J=7.8$ $\mathrm{Hz}), 7.42(1 \mathrm{H}, \mathrm{d}, J=1.2 \mathrm{~Hz}), 7.37(1 \mathrm{H}, \mathrm{d}, J=7.8 \mathrm{~Hz}), 7.28(3 \mathrm{H}$, $\mathrm{m}), 7.08(1 \mathrm{H}, \mathrm{d}, J=1.8 \mathrm{~Hz}), 7.01(1 \mathrm{H}, \mathrm{m}), 6.85(2 \mathrm{H}, \mathrm{m}), 6.60(1 \mathrm{H}$, $\mathrm{dd}, J=1.8,7.8 \mathrm{~Hz}), 5.79(1 \mathrm{H}, \mathrm{s}), 4.64(1 \mathrm{H}, \mathrm{d}, J=12.0 \mathrm{~Hz}), 4.32$ $(1 \mathrm{H}, \mathrm{d}, J=12.6 \mathrm{~Hz}), 4.26(1 \mathrm{H}, \mathrm{m}), 4.17(1 \mathrm{H}, \mathrm{m}), 3.94(1 \mathrm{H}, \mathrm{d}, J=$ $10.8 \mathrm{~Hz}), 3.88(3 \mathrm{H}, \mathrm{s}), 3.80(1 \mathrm{H}, \mathrm{m}), 3.69(1 \mathrm{H}, \mathrm{m}), 3.62(3 \mathrm{H}, \mathrm{s})$, $3.48(1 \mathrm{H}, \mathrm{m}), 3.04(1 \mathrm{H}, \mathrm{m}), 2.95(1 \mathrm{H}, \mathrm{m}), 2.66(2 \mathrm{H}, \mathrm{m}), 1.86(3 \mathrm{H}$, $\mathrm{m}), 1.66(1 \mathrm{H}, \mathrm{m}) .{ }^{13} \mathrm{C} \mathrm{NMR}\left(P y-d_{5}\right): \delta 193.3(\mathrm{C}=\mathrm{O}), 188.9(\mathrm{C}=\mathrm{O})$, $180.8(\mathrm{C}=\mathrm{O}), 179.3(\mathrm{C}=\mathrm{O}), 158.7\left(\mathrm{C},{ }^{1} J_{\mathrm{C}-\mathrm{F}}=239.1 \mathrm{~Hz}\right), 149.6$ (C), 149.5 (C), 148.1 (C), 147.8 (C), 143.8 (C), 140.1 (C), 139.6 $(\mathrm{CH}), 137.1(\mathrm{CH}), 132.0(\mathrm{C}), 129.6(\mathrm{C}), 129.4(\mathrm{C}), 127.1(\mathrm{C}), 122.4$ $(\mathrm{CH}), 120.8(\mathrm{CH}), 117.7(\mathrm{CH}), 117.2(\mathrm{CH}), 115.9\left(\mathrm{CH},{ }^{2} J_{\mathrm{C}-\mathrm{F}}=22.5\right.$ $\mathrm{Hz}), 114.3\left(\mathrm{CH},{ }^{2} J_{\mathrm{C}-\mathrm{F}}=24 \mathrm{~Hz}\right), 112.9(\mathrm{CH}), 112.0(\mathrm{CH}), 111.0$ $\left(\mathrm{CH},{ }^{3} J_{\mathrm{C}-\mathrm{F}}=6.45 \mathrm{~Hz}\right), 110.7(\mathrm{CH}), 101.0(\mathrm{CH}), 84.4(\mathrm{C}), 76.6(\mathrm{CH})$, 75.5 (C), $74.9(\mathrm{CH}), 74.6(\mathrm{C}), 64.1(\mathrm{CH}), 62.6(\mathrm{CH}), 56.5(\mathrm{OMe})$, 56.0 (OMe), $55.7\left(\mathrm{CH}_{2}\right), 52.5(\mathrm{CH}), 51.7(\mathrm{CH}), 48.2\left(\mathrm{CH}_{2}\right), 36.9$ $\left(\mathrm{CH}_{2}\right), 31.1\left(\mathrm{CH}_{2}\right), 28.0\left(\mathrm{CH}_{2}\right)$. MS [ESI-MS, positive mode]: found $m / z 953[\mathrm{M}+\mathrm{Na}]^{+}$. HRMS [ESI-MS, positive mode]: MF: $\mathrm{C}_{44} \mathrm{H}_{40} \mathrm{~N}_{4} \mathrm{O}_{8}$ IFS; found $m / z$ 953.1488 [M $\left.+\mathrm{Na}\right]^{+}$[calcd. 953.1493].

\section{Spectral data of compound $6 \mathrm{Bb}( \pm)$}

Obtained as white solid; yield: $37 \%$ (587 mg); mp: $220-222{ }^{\circ} \mathrm{C} ; R_{\mathrm{f}}$ $0.48\left(5 \% \mathrm{MeOH}\right.$ in $\left.\mathrm{CHCl}_{3}\right) ;{ }^{1} \mathrm{H}$ NMR $\left(P y-d_{5}\right): \delta 12.25(1 \mathrm{H}, \mathrm{s},-\mathrm{NH})$, $11.88(1 \mathrm{H}, \mathrm{s},-\mathrm{NH}), 8.31(1 \mathrm{H}, \mathrm{s}), 7.59(1 \mathrm{H}, \mathrm{d}, J=7.8 \mathrm{~Hz}), 7.30(1 \mathrm{H}$, $\mathrm{d}, J=7.8 \mathrm{~Hz}), 7.14(1 \mathrm{H}, \mathrm{d}, J=7.8 \mathrm{~Hz}), 7.09(1 \mathrm{H}, \mathrm{d}, J=8.4 \mathrm{~Hz})$, $7.06(1 \mathrm{H}, \mathrm{s}), 7.02(1 \mathrm{H}, \mathrm{s}), 6.96(1 \mathrm{H}, \mathrm{m}), 6.81(2 \mathrm{H}, \mathrm{m}), 6.76(2 \mathrm{H}, \mathrm{d}$, $J=7.8 \mathrm{~Hz}), 5.82(1 \mathrm{H}, \mathrm{s}), 4.32(1 \mathrm{H}, \mathrm{d}, J=12.0 \mathrm{~Hz}), 4.25(1 \mathrm{H}, \mathrm{m})$, $4.21(1 \mathrm{H}, \mathrm{d}, J=12.0 \mathrm{~Hz}), 4.14(1 \mathrm{H}, \mathrm{m}), 3.93(1 \mathrm{H}, \mathrm{d}, J=10.8 \mathrm{~Hz})$, $3.83(1 \mathrm{H}, \mathrm{m}), 3.73(3 \mathrm{H}, \mathrm{s}), 3.71(1 \mathrm{H}, \mathrm{m}), 3.65(1 \mathrm{H}, \mathrm{m}), 3.63(3 \mathrm{H}$, $\mathrm{s}), 3.12(1 \mathrm{H}, \mathrm{d}, J=12.0 \mathrm{~Hz}), 2.99(1 \mathrm{H}, \mathrm{m}), 2.63(2 \mathrm{H}, \mathrm{m}), 1.84(1 \mathrm{H}$, $\mathrm{m}), 1.76(1 \mathrm{H}, \mathrm{m}), 1.67(2 \mathrm{H}, \mathrm{m}) .{ }^{13} \mathrm{C} \mathrm{NMR}\left(P y-d_{5}\right): \delta 190.4(\mathrm{C}=\mathrm{O})$, $189.2(\mathrm{C}=\mathrm{O}), 181.0(\mathrm{C}=\mathrm{O}), 179.6(\mathrm{C}=\mathrm{O}), 158.6\left(\mathrm{C},{ }^{1} J_{\mathrm{C}-\mathrm{F}}=237.0\right.$ Hz), 149.1 (C), 149.0 (C), 147.8 (C), 147.4 (C), 143.5 (C), 139.8 (C), $139.1(\mathrm{CH}), 137.4(\mathrm{CH}), 131.1(\mathrm{C}), 130.1(\mathrm{C}), 128.3(\mathrm{C}), 127.0(\mathrm{C})$, $120.7(\mathrm{CH}), 120.3(\mathrm{CH}), 117.2(\mathrm{CH}), 117.1(\mathrm{CH}), 116.2\left(\mathrm{CH},{ }^{2} J_{\mathrm{C}-\mathrm{F}}\right.$ $=24.0 \mathrm{~Hz}), 115.0\left(\mathrm{CH},{ }^{2} J_{\mathrm{C}-\mathrm{F}}=24 \mathrm{~Hz}\right), 112.6(\mathrm{CH}), 112.5(2 \times$ $\mathrm{CH}), 111.2\left(\mathrm{CH},{ }^{3} J_{\mathrm{C}-\mathrm{F}}=7.5 \mathrm{~Hz}\right), 101.7(\mathrm{CH}), 84.5(\mathrm{C}), 76.4(\mathrm{CH})$, 76.0 (C), $75.3(\mathrm{C}), 73.6(\mathrm{CH}), 64.8(\mathrm{CH}), 63.5(\mathrm{CH}), 56.1$ (OMe), 56.0 (OMe), $55.5\left(\mathrm{CH}_{2}\right), 51.9(\mathrm{CH}), 51.0(\mathrm{CH}), 48.2\left(\mathrm{CH}_{2}\right), 37.1$ $\left(\mathrm{CH}_{2}\right), 31.4\left(\mathrm{CH}_{2}\right), 28.3\left(\mathrm{CH}_{2}\right)$. MS [ESI-MS, positive mode]: found $m / z 953[\mathrm{M}+\mathrm{Na}]^{+}$. HRMS [ESI-MS, positive mode]: MF: $\mathrm{C}_{44} \mathrm{H}_{40} \mathrm{~N}_{4} \mathrm{O}_{8} \mathrm{IFS}$; found $m / z$ 953.1487 [M $\left.+\mathrm{Na}\right]^{+}$[calcd. 953.1493].

\section{Spectral data of compound $6 \mathrm{Ca}( \pm)$}

Obtained as white solid; yield: $31 \%$ (455 mg); mp: $180-182{ }^{\circ} \mathrm{C} ; R_{\mathrm{f}}$ 0.54 (5\% $\mathrm{MeOH}$ in $\mathrm{CHCl}_{3}$ ); IR (KBr, $\left.\nu_{\max } \mathrm{cm}^{-1}\right)$ : 3514, 3200, 1713, 1610, 1515; ${ }^{1} \mathrm{H}$ NMR $\left(P y-d_{5}\right): \delta 11.61(1 \mathrm{H}, \mathrm{s},-\mathrm{NH}), 11.39$ $(1 \mathrm{H}, \mathrm{s},-\mathrm{NH}), 8.29(1 \mathrm{H}, \mathrm{s}), 7.76(1 \mathrm{H}, \mathrm{d}, J=7.8 \mathrm{~Hz}), 7.57(1 \mathrm{H}, \mathrm{m})$, $7.34(2 \mathrm{H}, \mathrm{m}), 7.28(2 \mathrm{H}, \mathrm{m}), 7.18(1 \mathrm{H}, \mathrm{d}, J=7.8 \mathrm{~Hz}), 7.13(1 \mathrm{H}, \mathrm{s})$, $6.88(1 \mathrm{H}, \mathrm{d}, J=7.8 \mathrm{~Hz}), 6.80(1 \mathrm{H}, \mathrm{d}, J=7.8 \mathrm{~Hz}), 6.77(1 \mathrm{H}, \mathrm{d}, J=$ $8.4 \mathrm{~Hz}), 5.74(1 \mathrm{H}, \mathrm{s}), 4.57(1 \mathrm{H}, \mathrm{d}, J=12.0 \mathrm{~Hz}), 4.38(1 \mathrm{H}, \mathrm{m}), 4.27$ $(1 \mathrm{H}, \mathrm{m}), 4.20(1 \mathrm{H}, \mathrm{m}), 3.92(1 \mathrm{H}, \mathrm{d}, J=10.8 \mathrm{~Hz}), 3.82(1 \mathrm{H}, \mathrm{m})$, $3.80(3 \mathrm{H}, \mathrm{s}), 3.67(3 \mathrm{H}, \mathrm{s}), 3.64(1 \mathrm{H}, \mathrm{m}), 3.57(1 \mathrm{H}, \mathrm{m}), 3.08(1 \mathrm{H}, \mathrm{d}$, $J=11.4 \mathrm{~Hz}), 2.96(1 \mathrm{H}, \mathrm{m}), 2.71(1 \mathrm{H}, \mathrm{m}), 2.61(1 \mathrm{H}, \mathrm{m}), 1.82(3 \mathrm{H}$, $\mathrm{m}), 1.66(1 \mathrm{H}, \mathrm{m}) .{ }^{13} \mathrm{C} \mathrm{NMR}\left(P y-d_{5}\right): \delta 192.8(\mathrm{C}=\mathrm{O}), 189.0(\mathrm{C}=\mathrm{O})$, 
$180.5(\mathrm{C}=\mathrm{O}), 179.3(\mathrm{C}=\mathrm{O}), 149.9(\mathrm{C}), 149.5(\mathrm{C}), 148.0$ (C), 147.7 (C), 143.7 (C), 142.6 (C), $139.4(\mathrm{CH}), 137.0(\mathrm{CH}), 131.9$ (C), 130.0 (CH), 129.8 (C), 129.3 (C), 127.2 (C), $126.9(\mathrm{CH}), 126.6$ (C), 121.9 $(\mathrm{CH}), 120.9(\mathrm{CH}), 117.5(\mathrm{CH}), 117.3(\mathrm{CH}), 112.8(\mathrm{CH}), 112.0(\mathrm{CH})$, $111.8(\mathrm{CH}), 111.2(\mathrm{CH}), 101.1(\mathrm{CH}), 84.5(\mathrm{C}), 76.7(\mathrm{CH}), 75.2(2 \times$ C), $74.4(\mathrm{CH}), 64.6(\mathrm{CH}), 62.4(\mathrm{CH}), 56.3$ (OMe), 56.1 (OMe), 55.3 $\left(\mathrm{CH}_{2}\right), 52.4(\mathrm{CH}), 51.4(\mathrm{CH}), 48.2\left(\mathrm{CH}_{2}\right), 36.9\left(\mathrm{CH}_{2}\right), 31.0\left(\mathrm{CH}_{2}\right)$, $27.9\left(\mathrm{CH}_{2}\right)$. MS [ESI-MS, positive mode]: found $m / z 947[\mathrm{M}+\mathrm{H}]^{+}$. HRMS [ESI-MS, positive mode]: MF: $\mathrm{C}_{44} \mathrm{H}_{40} \mathrm{ClIN}_{4} \mathrm{O}_{8} \mathrm{~S}$; found $\mathrm{m} / \mathrm{z}$ 947.1375 $[\mathrm{M}+\mathrm{H}]^{+}$[calcd. 947.1378].

\section{Spectral data of compound $6 \mathrm{Cb}( \pm)$}

Obtained as white solid; yield: $35 \%$ (549 mg); mp: $230-232{ }^{\circ} \mathrm{C} ; R_{\mathrm{f}}$ 0.46 (5\% MeOH in $\left.\mathrm{CHCl}_{3}\right) ;{ }^{1} \mathrm{H}$ NMR $\left(P y-d_{5}\right): \delta 12.23(1 \mathrm{H}, \mathrm{s},-\mathrm{NH})$, $12.06(1 \mathrm{H}, \mathrm{s},-\mathrm{NH}), 8.30(1 \mathrm{H}, \mathrm{s}), 7.59(1 \mathrm{H}, \mathrm{m}), 7.55$ (1H, dd, $J=$ 1.8, 8.4 Hz), 7.17 (1H, dd, $J=1.8,8.4 \mathrm{~Hz}), 7.10(2 \mathrm{H}, \mathrm{dd}, J=3.0$, $8.4 \mathrm{~Hz}), 7.02(2 \mathrm{H}, \mathrm{m}), 6.79(1 \mathrm{H}, \mathrm{d}, J=8.4 \mathrm{~Hz}), 6.75(2 \mathrm{H}, \mathrm{m}), 6.68$ $(1 \mathrm{H}, \mathrm{d}, J=7.8 \mathrm{~Hz}), 5.80(1 \mathrm{H}, \mathrm{s}), 4.28(2 \mathrm{H}, \mathrm{d}, J=12.0 \mathrm{~Hz}), 4.18$ $(1 \mathrm{H}, \mathrm{m}), 4.14(1 \mathrm{H}, \mathrm{m}), 3.92(1 \mathrm{H}, \mathrm{d}, J=10.8 \mathrm{~Hz}), 3.82(2 \mathrm{H}, \mathrm{t}, J=$ $10.8 \mathrm{~Hz}), 3.68(3 \mathrm{H}, \mathrm{s}), 3.64(3 \mathrm{H}, \mathrm{s}), 3.62(1 \mathrm{H}, \mathrm{m}), 3.10(1 \mathrm{H}, \mathrm{m})$, $2.99(1 \mathrm{H}, \mathrm{m}), 2.67(1 \mathrm{H}, \mathrm{m}), 2.60(1 \mathrm{H}, \mathrm{m}), 1.85(1 \mathrm{H}, \mathrm{m}), 1.79(1 \mathrm{H}$, $\mathrm{m}), 1.70(2 \mathrm{H}, \mathrm{m}) .{ }^{13} \mathrm{C}$ NMR $\left(P y-d_{5}\right): \delta 189.8(2 \times \mathrm{C}=\mathrm{O}), 180.8$ $(\mathrm{C}=\mathrm{O}), 179.5$ (C=O), 149.0 (C), 148.9 (C), 147.7 (C), 147.3 (C), 143.5 (C), $142.4(\mathrm{C}), 139.1(\mathrm{CH}), 137.4(\mathrm{CH}), 131.1(\mathrm{C}), 130.1(\mathrm{C})$, 129.9 (CH), 128.5 (C), $127.6(\mathrm{CH}), 127.0$ (C), 126.7 (C), 120.5 $(\mathrm{CH}), 120.1(\mathrm{CH}), 117.1(\mathrm{CH}), 117.0(\mathrm{CH}), 112.8(\mathrm{CH}), 112.6(\mathrm{CH})$, $112.5(\mathrm{CH}), 111.8(\mathrm{CH}), 101.7(\mathrm{CH}), 84.5(\mathrm{C}), 76.3(\mathrm{CH}), 76.1(\mathrm{C})$, $75.3(\mathrm{C}), 73.3(\mathrm{CH}), 65.5(\mathrm{CH}), 63.6(\mathrm{CH}), 56.13$ (OMe), 56.06 (OMe), $55.5\left(\mathrm{CH}_{2}\right), 51.7(\mathrm{CH}), 50.9(\mathrm{CH}), 48.2\left(\mathrm{CH}_{2}\right), 37.1\left(\mathrm{CH}_{2}\right)$, $31.3\left(\mathrm{CH}_{2}\right), 28.4\left(\mathrm{CH}_{2}\right)$. MS [ESI-MS, positive mode]: found $\mathrm{m} / \mathrm{z}$ $947[\mathrm{M}+\mathrm{H}]^{+}$. HRMS [ESI-MS, positive mode]: MF: $\mathrm{C}_{44} \mathrm{H}_{40^{-}}$ $\mathrm{ClIN}_{4} \mathrm{O}_{8} \mathrm{~S}$; found $\mathrm{m} / \mathrm{z}$ 969.1193 [M $\left.+\mathrm{Na}\right]+[$ calcd. 969.1198].

\section{Spectral data of compound $6 \mathrm{Da}( \pm)$}

Obtained as white solid; yield: $32 \%$ (452 mg); mp: $202-204{ }^{\circ} \mathrm{C} ; R_{\mathrm{f}}$ 0.55 (5\% MeOH in $\mathrm{CHCl}_{3}$ ); IR (KBr, $\left.\nu_{\max } \mathrm{cm}^{-1}\right)$ : 3411, 2930, 1721, 1604, 1518; ${ }^{1} \mathrm{H}$ NMR $\left(P y-d_{5}\right): \delta 11.26(1 \mathrm{H}, \mathrm{s},-\mathrm{NH}), 10.63$ $(1 \mathrm{H}, \mathrm{s},-\mathrm{NH}), 7.43(1 \mathrm{H}, \mathrm{s}), 7.28(2 \mathrm{H}, \mathrm{t}, J=8.4 \mathrm{~Hz}), 7.23(2 \mathrm{H}, \mathrm{m})$, $7.10(1 \mathrm{H}, \mathrm{d}, J=2.4 \mathrm{~Hz}), 7.06(1 \mathrm{H}, \mathrm{dd}, J=1.2,7.8 \mathrm{~Hz}), 7.01(1 \mathrm{H}$, $\mathrm{dd}, J=1.2,7.8 \mathrm{~Hz}), 6.86(1 \mathrm{H}, \mathrm{d}, J=7.8 \mathrm{~Hz}), 6.81(1 \mathrm{H}, \mathrm{dd}, J=2.4$, $8.4 \mathrm{~Hz}), 6.75(1 \mathrm{H}, \mathrm{s}), 5.72(1 \mathrm{H}, \mathrm{s}), 4.38(3 \mathrm{H}, \mathrm{m}), 4.28(1 \mathrm{H}, \mathrm{m}), 3.95$ $(1 \mathrm{H}, \mathrm{m}), 3.81(1 \mathrm{H}, \mathrm{m}), 3.75(3 \mathrm{H}, \mathrm{s}), 3.74(3 \mathrm{H}, \mathrm{s}), 3.71(2 \mathrm{H}, \mathrm{m})$, $3.64(3 \mathrm{H}, \mathrm{s}), 3.17(1 \mathrm{H}, \mathrm{m}), 3.00(1 \mathrm{H}, \mathrm{m}), 2.88(1 \mathrm{H}, \mathrm{m}), 2.62(1 \mathrm{H}$, m), $2.23(3 \mathrm{H}, \mathrm{s}), 2.15(3 \mathrm{H}, \mathrm{s}), 1.88(1 \mathrm{H}, \mathrm{m}), 1.79(1 \mathrm{H}, \mathrm{m}), 1.70$ $(2 \mathrm{H}, \mathrm{m}) \cdot{ }^{13} \mathrm{C}$ NMR $\left(P y-d_{5}\right): \delta 194.0(\mathrm{C}=\mathrm{O}), 187.9(\mathrm{C}=\mathrm{O}), 180.9$ $(\mathrm{C}=\mathrm{O}), 180.4(\mathrm{C}=\mathrm{O}), 155.4(\mathrm{C}), 149.4(\mathrm{C}), 149.1$ (C), 147.7 (C), 147.5 (C), 140.0 (C), 136.8 (C), 132.3 (CH), 132.0 (C), 130.6 (C), 130.5 (C), 127.8 (C), $126.5(\mathrm{CH}), 124.2(\mathrm{C}), 121.3(\mathrm{CH}), 121.0$ $(\mathrm{CH}), 119.0(\mathrm{C}), 117.3(\mathrm{CH}), 117.1(\mathrm{CH}), 115.0(\mathrm{CH}), 114.5(\mathrm{CH})$, $112.4(\mathrm{CH}), 111.7(\mathrm{CH}), 110.8(\mathrm{CH}), 101.9(\mathrm{CH}), 77.1(\mathrm{CH}), 75.5$ (C), $74.8(\mathrm{C}), 72.9(\mathrm{CH}), 66.1(\mathrm{CH}), 61.5(\mathrm{CH}), 56.13(2 \times \mathrm{OMe})$, 56.09 (OMe), $55.2\left(\mathrm{CH}_{2}\right), 52.5(\mathrm{CH}), 51.1(\mathrm{CH}), 48.5\left(\mathrm{CH}_{2}\right), 36.7$ $\left(\mathrm{CH}_{2}\right), 30.6\left(\mathrm{CH}_{2}\right), 27.5\left(\mathrm{CH}_{2}\right), 21.1\left(\mathrm{CH}_{3}\right), 17.2\left(\mathrm{CH}_{3}\right)$. MS [ESIMS, positive mode]: found $m / z 845[\mathrm{M}+\mathrm{H}]^{+}$. HRMS [ESI-MS, positive mode]: $\mathrm{MF}: \mathrm{C}_{47} \mathrm{H}_{48} \mathrm{~N}_{4} \mathrm{O}_{9} \mathrm{~S}$; found $m / z$ 845.3238 [M + $\mathrm{H}^{+}$[calcd. 845.3220].

\section{Spectral data of compound 6Db $( \pm)$}

Obtained as white solid; yield: $36 \%$ (508 mg); mp: $182-184{ }^{\circ} \mathrm{C} ; R_{\mathrm{f}}$ $0.47\left(5 \% \mathrm{MeOH}\right.$ in $\left.\mathrm{CHCl}_{3}\right) ;{ }^{1} \mathrm{H}$ NMR $\left(P y-d_{5}\right): \delta 11.83(1 \mathrm{H}, \mathrm{s},-\mathrm{NH})$, $11.79(1 \mathrm{H}, \mathrm{s},-\mathrm{NH}), 7.47(1 \mathrm{H}, \mathrm{s}), 7.18(1 \mathrm{H}, \mathrm{dd}, J=1.2,7.8 \mathrm{~Hz})$, $7.12(2 \mathrm{H}, \mathrm{m}), 7.07(2 \mathrm{H}, \mathrm{m}), 6.84(3 \mathrm{H}, \mathrm{m}), 6.77(1 \mathrm{H}, \mathrm{dd}, J=2.4,8.4$ $\mathrm{Hz}), 6.69(1 \mathrm{H}, \mathrm{s}), 5.87(1 \mathrm{H}, \mathrm{d}, J=1.2 \mathrm{~Hz}), 4.33(2 \mathrm{H}, \mathrm{m}), 4.27(1 \mathrm{H}$, $\mathrm{d}, J=12.6 \mathrm{~Hz}), 4.19(1 \mathrm{H}, \mathrm{m}), 3.98(1 \mathrm{H}, \mathrm{d}, J=10.2 \mathrm{~Hz}), 3.90(1 \mathrm{H}$, $\mathrm{m}), 3.80(1 \mathrm{H}, \mathrm{t}, J=10.2 \mathrm{~Hz}), 3.75(1 \mathrm{H}, \mathrm{d}, J=10.2 \mathrm{~Hz}), 3.68$ $(3 \mathrm{H}, \mathrm{s}), 3.64(3 \mathrm{H}, \mathrm{s}), 3.54(3 \mathrm{H}, \mathrm{s}), 3.17(1 \mathrm{H}, \mathrm{m}), 3.04(1 \mathrm{H}, \mathrm{m}), 2.81$ $(1 \mathrm{H}, \mathrm{m}), 2.64(1 \mathrm{H}, \mathrm{m}), 2.26(3 \mathrm{H}, \mathrm{s}), 2.10(3 \mathrm{H}, \mathrm{s}), 1.87(1 \mathrm{H}, \mathrm{m})$, $1.79(1 \mathrm{H}, \mathrm{m}), 1.69(2 \mathrm{H}, \mathrm{m}) .{ }^{13} \mathrm{C}$ NMR $\left(P y-d_{5}\right): \delta 191.0(\mathrm{C}=\mathrm{O})$, $189.3(\mathrm{C}=\mathrm{O}), 181.2(\mathrm{C}=\mathrm{O}), 180.6(\mathrm{C}=\mathrm{O}), 155.4(\mathrm{C}), 149.1$ (C), 149.0 (C), 147.7 (C), 147.4 (C), 140.0 (C), 137.1 (C), 132.3 (CH), 131.6 (C), 130.7 (C), $130.4(\mathrm{C}), 127.9$ (C), $126.5(\mathrm{CH}), 124.3$ (C), $120.7(\mathrm{CH}), 120.3(\mathrm{CH}), 119.1(\mathrm{C}), 117.2(\mathrm{CH}), 116.9(\mathrm{CH})$, $115.3(\mathrm{CH}), 114.1(\mathrm{CH}), 112.6(\mathrm{CH}), 112.4(\mathrm{CH}), 110.7(\mathrm{CH}), 101.4$ $(\mathrm{CH}), 76.6(\mathrm{CH}), 76.1(\mathrm{C}), 75.5(\mathrm{C}), 73.4(\mathrm{CH}), 65.2(\mathrm{CH}), 63.3$ $(\mathrm{CH}), 56.1$ (OMe), 56.0 (OMe), $55.8(\mathrm{OMe}), 55.0\left(\mathrm{CH}_{2}\right), 51.8(\mathrm{CH})$, $50.7(\mathrm{CH}), 48.4\left(\mathrm{CH}_{2}\right), 36.9\left(\mathrm{CH}_{2}\right), 31.3\left(\mathrm{CH}_{2}\right), 28.1\left(\mathrm{CH}_{2}\right), 21.3$ $\left(\mathrm{CH}_{3}\right), 17.2\left(\mathrm{CH}_{3}\right)$. MS [ESI-MS, positive mode]: found $\mathrm{m} / z 845[\mathrm{M}$ $+\mathrm{H}]^{+}$. HRMS [ESI-MS, positive mode]: MF: $\mathrm{C}_{47} \mathrm{H}_{48} \mathrm{~N}_{4} \mathrm{O}_{9} \mathrm{~S}$; found $m / z$ 845.3209 [M + H $]^{+}$[calcd. 845.3220].

\section{Spectral data of compound $6 \mathrm{Ea}( \pm)$}

Obtained as white solid; yield: $31 \%$ (441 mg); mp: $172-174{ }^{\circ} \mathrm{C} ; R_{\mathrm{f}}$ 0.51 (5\% MeOH in $\left.\mathrm{CHCl}_{3}\right) ;{ }^{1} \mathrm{H}$ NMR $\left(P y-d_{5}\right): \delta 11.16(1 \mathrm{H}, \mathrm{s},-\mathrm{NH})$, 10.69 (1H, s, $-\mathrm{NH}), 7.46(1 \mathrm{H}, \mathrm{s}), 7.30(2 \mathrm{H}, \mathrm{m}), 7.28(1 \mathrm{H}, \mathrm{s}), 7.25$ $(2 \mathrm{H}, \mathrm{d}, J=9.6 \mathrm{~Hz}), 7.08(1 \mathrm{H}, \mathrm{d}, J=7.8 \mathrm{~Hz}), 7.03(1 \mathrm{H}, \mathrm{d}, J=7.8$ $\mathrm{Hz}), 6.95(1 \mathrm{H}, \mathrm{d}, J=7.8 \mathrm{~Hz}), 6.83(1 \mathrm{H}, \mathrm{d}, J=7.8 \mathrm{~Hz}), 6.73(1 \mathrm{H}, \mathrm{s})$, $5.73(1 \mathrm{H}, \mathrm{s}), 4.37(3 \mathrm{H}, \mathrm{m}), 3.95(1 \mathrm{H}, \mathrm{d}, J=10.2 \mathrm{~Hz}), 3.87(1 \mathrm{H}, \mathrm{m})$, $3.81(1 \mathrm{H}, \mathrm{m}), 3.76(3 \mathrm{H}, \mathrm{s}), 3.73(3 \mathrm{H}, \mathrm{s}), 3.71(1 \mathrm{H}, \mathrm{m}), 3.16$ $(1 \mathrm{H}, \mathrm{m}), 3.01(1 \mathrm{H}, \mathrm{m}), 2.86(1 \mathrm{H}, \mathrm{m}), 2.63(1 \mathrm{H}, \mathrm{m}), 2.24(3 \mathrm{H}, \mathrm{s})$, $2.15(6 \mathrm{H}, \mathrm{s}), 2.01(1 \mathrm{H}, \mathrm{m}), 1.90(1 \mathrm{H}, \mathrm{m}), 1.82(2 \mathrm{H}, \mathrm{m}), 1.69$ (1H, m). ${ }^{13} \mathrm{C}$ NMR $\left(P y-d_{5}\right): \delta 192.6(\mathrm{C}=\mathrm{O}), 187.8(\mathrm{C}=\mathrm{O}), 180.5$ (C=O), 179.9 (C=O), 148.7 (C), 148.5 (C), 147.1 (C), 146.8 (C), 140.4 (C), 139.3 (C), $131.7(\mathrm{CH}), 131.4(\mathrm{C}), 130.2(3 \times \mathrm{C}), 129.9$ (C), $129.6(\mathrm{CH}), 127.5(\mathrm{CH}), 126.0(\mathrm{C}), 125.9(\mathrm{CH}), 120.5(\mathrm{CH})$, $120.3(\mathrm{CH}), 118.4(\mathrm{C}), 116.7(\mathrm{CH}), 116.5(\mathrm{CH}), 111.8(\mathrm{CH}), 111.4$ $(\mathrm{CH}), 109.7(\mathrm{CH}), 101.2(\mathrm{CH}), 76.2(\mathrm{CH}), 75.0(\mathrm{C}), 74.1(\mathrm{C}), 72.6$ $(\mathrm{CH}), 65.0(\mathrm{CH}), 61.4(\mathrm{CH}), 55.5(2 \times \mathrm{OMe}), 54.4\left(\mathrm{CH}_{2}\right), 51.7$ $(\mathrm{CH}), 50.5(\mathrm{CH}), 48.0\left(\mathrm{CH}_{2}\right), 36.1\left(\mathrm{CH}_{2}\right), 30.2\left(\mathrm{CH}_{2}\right), 27.0\left(\mathrm{CH}_{2}\right)$, $20.7\left(\mathrm{CH}_{3}\right), 20.6\left(\mathrm{CH}_{3}\right), 16.6\left(\mathrm{CH}_{3}\right)$. MS [ESI-MS, positive mode]: found $m / z 829[\mathrm{M}+\mathrm{H}]^{+}$. HRMS [ESI-MS, positive mode]: MF: $\mathrm{C}_{47} \mathrm{H}_{49} \mathrm{~N}_{4} \mathrm{O}_{8} \mathrm{~S}$; found $\mathrm{m} / z$ 829.3268 [M+ H$]^{+}$[calcd. 829.3271].

\section{Spectral data of compound $6 \mathrm{~Eb}( \pm)$}

Obtained as white solid; yield: $36 \%(512 \mathrm{mg}) ; \mathrm{mp}: 169-171{ }^{\circ} \mathrm{C} ; R_{\mathrm{f}}$ 0.45 (5\% $\mathrm{MeOH}$ in $\left.\mathrm{CHCl}_{3}\right)$; IR $\left(\mathrm{KBr}, \nu_{\max } \mathrm{cm}^{-1}\right)$ : 3404, 2926, 1712, 1605, 1517, 1485; ${ }^{1} \mathrm{H}$ NMR $\left(P y-d_{5}\right): \delta 7.41(1 \mathrm{H}, \mathrm{s}), 7.17(2 \mathrm{H}$, $\mathrm{m}), 7.09(3 \mathrm{H}, \mathrm{m}), 6.90(1 \mathrm{H}, \mathrm{d}, J=7.8 \mathrm{~Hz}), 6.82(2 \mathrm{H}, \mathrm{m}), 6.78(1 \mathrm{H}$, $\mathrm{d}, J=7.8 \mathrm{~Hz}), 6.64(1 \mathrm{H}, \mathrm{s}), 5.85(1 \mathrm{H}, \mathrm{s}), 4.27(3 \mathrm{H}, \mathrm{m}), 4.16$ $(1 \mathrm{H}, \mathrm{m}), 3.93(1 \mathrm{H}, \mathrm{d}, J=9.6 \mathrm{~Hz}), 3.83(2 \mathrm{H}, \mathrm{q}, J=9.6,21 \mathrm{~Hz}), 3.70$ $(1 \mathrm{H}, \mathrm{d}, J=9.6 \mathrm{~Hz}), 3.63(3 \mathrm{H}, \mathrm{s}), 3.58(3 \mathrm{H}, \mathrm{s}), 3.13(1 \mathrm{H}, \mathrm{m})$, $2.99(1 \mathrm{H}, \mathrm{m}), 2.78(1 \mathrm{H}, \mathrm{m}), 2.59(1 \mathrm{H}, \mathrm{m}), 2.21(3 \mathrm{H}, \mathrm{s}), 2.05(3 \mathrm{H}$, s), $2.00(3 \mathrm{H}, \mathrm{s}), 1.84(1 \mathrm{H}, \mathrm{m}), 1.75(2 \mathrm{H}, \mathrm{m}), 1.64(1 \mathrm{H}, \mathrm{m}) \cdot{ }^{13} \mathrm{C}$ 
NMR $\left(P y-d_{5}\right): \delta 190.6(\mathrm{C}=\mathrm{O}), 188.8(\mathrm{C}=\mathrm{O}), 180.8(\mathrm{C}=\mathrm{O}), 180.1$ (C=O), 148.6 (C), 148.4 (C), 147.2 (C), 146.8 (C), 140.7 (C), 139.5 (C), $131.8(\mathrm{CH}), 131.1$ (C), $130.2(2 \times \mathrm{C}), 130.1$ (C), 129.8 (C), $129.7(\mathrm{CH}), 127.5(\mathrm{CH}), 126.0(\mathrm{C}), 125.9(\mathrm{CH}), 120.1(\mathrm{CH}), 119.7$ $(\mathrm{CH}), 118.5(\mathrm{C}), 116.6(\mathrm{CH}), 116.4(\mathrm{CH}), 111.9(\mathrm{CH}), 111.7(\mathrm{CH})$, $109.7(\mathrm{CH}), 100.7(\mathrm{CH}), 76.1(\mathrm{CH}), 75.5(\mathrm{C}), 74.7(\mathrm{C}), 73.0(\mathrm{CH})$, $64.5(\mathrm{CH}), 62.5(\mathrm{CH}), 55.5(\mathrm{OMe}), 55.4(\mathrm{OMe}), 54.5\left(\mathrm{CH}_{2}\right), 51.1$ $(\mathrm{CH}), 50.2(\mathrm{CH}), 47.9\left(\mathrm{CH}_{2}\right), 36.3\left(\mathrm{CH}_{2}\right), 30.7\left(\mathrm{CH}_{2}\right), 27.5\left(\mathrm{CH}_{2}\right)$, $20.8\left(\mathrm{CH}_{3}\right), 20.7\left(\mathrm{CH}_{3}\right), 16.7\left(\mathrm{CH}_{3}\right)$. MS [ESI-MS, positive mode]: found $m / z 829[\mathbf{M}+\mathbf{H}]^{+}$. HRMS [ESI-MS, positive mode]: MF: $\mathrm{C}_{47} \mathrm{H}_{49} \mathrm{~N}_{4} \mathrm{O}_{8} \mathrm{~S}$; found $m / z$ 829.3268 [M+ H$]^{+}$[calcd. 829.3271].

\section{Spectral data of compound 7A $( \pm)$}

Obtained as white solid; yield: $54 \%$ (636 mg); mp: $192-194{ }^{\circ} \mathrm{C} ; R_{\mathrm{f}}$ 0.52 (5\% MeOH in $\mathrm{CHCl}_{3}$ ); UV absorption maximas $\left(\lambda_{\max } \mathrm{nm}\right.$, methanol) 314, 228; IR (KBr, $\left.\nu_{\max } \mathrm{cm}^{-1}\right): 3511,2960,1720,1607$, 1515; ${ }^{1} \mathrm{H}$ NMR $\left(P y-d_{5}\right): \delta 11.82(1 \mathrm{H}, \mathrm{s},-\mathrm{NH}), 7.87(2 \mathrm{H}, \mathrm{m}), 7.84$ $(2 \mathrm{H}, \mathrm{m}), 7.76(1 \mathrm{H}, \mathrm{t}, J=7.2 \mathrm{~Hz}), 7.69(1 \mathrm{H}, \mathrm{d}, J=7.2 \mathrm{~Hz}), 7.41(1 \mathrm{H}$, $\mathrm{t}, J=7.2 \mathrm{~Hz}), 7.37(1 \mathrm{H}, \mathrm{d}, J=1.2 \mathrm{~Hz}), 7.30(1 \mathrm{H}, \mathrm{t}, J=7.8 \mathrm{~Hz}), 7.16$ $(3 \mathrm{H}, \mathrm{m}), 7.02(1 \mathrm{H}, \mathrm{d}, J=7.8 \mathrm{~Hz}), 6.79(1 \mathrm{H}, \mathrm{dd}, J=1.2,8.4 \mathrm{~Hz})$, $6.75(1 \mathrm{H}, \mathrm{d}, J=8.4 \mathrm{~Hz}), 6.70(1 \mathrm{H}, \mathrm{s}), 6.03(1 \mathrm{H}, \mathrm{s}), 4.96(1 \mathrm{H}, \mathrm{m})$, $4.61(2 \mathrm{H}, \mathrm{m}), 4.45(1 \mathrm{H}, \mathrm{d}, J=11.4 \mathrm{~Hz}), 4.38(1 \mathrm{H}, \mathrm{m}), 4.01(1 \mathrm{H}$, $\mathrm{dd}, J=9.6,12 \mathrm{~Hz}), 3.71(3 \mathrm{H}, \mathrm{s}), 3.36(3 \mathrm{H}, \mathrm{s}), 3.14(1 \mathrm{H}, \mathrm{m}), 2.90$ $(1 \mathrm{H}, \mathrm{m}), 2.74(1 \mathrm{H}, \mathrm{m}), 2.54(1 \mathrm{H}, \mathrm{t}, J=7.2 \mathrm{~Hz}), 1.98(1 \mathrm{H}, \mathrm{m}), 1.90$ $(2 \mathrm{H}, \mathrm{m}), 1.78(1 \mathrm{H}, \mathrm{m}), 1.70(1 \mathrm{H}, \mathrm{m}), 1.64(1 \mathrm{H}, \mathrm{m}), 1.50(1 \mathrm{H}, \mathrm{m})$, $1.31(1 \mathrm{H}, \mathrm{m}) .{ }^{13} \mathrm{C}$ NMR $\left(P y-d_{5}\right): \delta 204.6(\mathrm{C}=\mathrm{O}), 197.4(\mathrm{C}=\mathrm{O})$, $186.5(\mathrm{C}=\mathrm{O}), 181.2(\mathrm{C}=\mathrm{O}), 149.3(\mathrm{C}), 148.1(\mathrm{C}), 147.7(\mathrm{C}), 147.2$ (C), 144.0 (C), 143.2 (C), 138.1 (C), 133.2 (C), 131.7 (CH), 131.5 (C), $131.3(\mathrm{C}), 129.9(\mathrm{CH}), 128.83(\mathrm{CH}), 128.79(\mathrm{CH}), 128.0(\mathrm{CH})$, 127.5 (C), 126.9 (C), $125.9(\mathrm{CH}), 123.7(\mathrm{CH}), 121.9(\mathrm{CH}), 121.8$ $(\mathrm{CH}), 121.7(\mathrm{CH}), 121.4(\mathrm{CH}), 117.1(\mathrm{CH}), 116.3(\mathrm{CH}), 113.2(\mathrm{CH})$, $112.3(\mathrm{CH}), 110.8(\mathrm{CH}), 103.0(\mathrm{CH}), 79.3(\mathrm{C}), 75.3(\mathrm{C}), 73.7(\mathrm{CH})$, $66.0(\mathrm{CH}), 64.6(\mathrm{CH}), 56.1(\mathrm{OMe}), 55.6(\mathrm{OMe}), 54.8(\mathrm{CH}), 52.6$ $(\mathrm{CH}), 51.8\left(\mathrm{CH}_{2}\right), 50.7(\mathrm{CH}), 48.6\left(\mathrm{CH}_{2}\right), 31.3\left(\mathrm{CH}_{2}\right), 29.5\left(\mathrm{CH}_{2}\right)$, $28.2\left(\mathrm{CH}_{2}\right), 26.7\left(\mathrm{CH}_{2}\right)$. MS [ESI-MS, positive mode]: found $\mathrm{m} / \mathrm{z}$ $804[\mathrm{M}+\mathrm{H}]^{+}, 826[\mathrm{M}+\mathrm{Na}]^{+}$. HRMS [ESI-MS, positive mode]: MF: $\mathrm{C}_{49} \mathrm{H}_{45} \mathrm{~N}_{3} \mathrm{O}_{8}$; found $m / z$ 826.3113 [M+Na] $]^{+}$[calcd. 826.3104].

\section{Spectral data of compound $7 \mathbf{B}( \pm)$}

Obtained as yellowish white solid; yield: 56\% (629 mg); mp: $163-165{ }^{\circ} \mathrm{C} ; R_{\mathrm{f}} 0.50$ (5\% $\mathrm{MeOH}$ in $\left.\mathrm{CHCl}_{3}\right) ;{ }^{1} \mathrm{H}$ NMR $\left(P y-d_{5}\right)$ : $\delta 12.17(1 \mathrm{H}, \mathrm{s},-\mathrm{NH}), 8.06(2 \mathrm{H}, \mathrm{d}, J=7.2 \mathrm{~Hz}), 7.88(1 \mathrm{H}, \mathrm{d}, J=8.4$ $\mathrm{Hz}), 7.84(1 \mathrm{H}, \mathrm{s}), 7.67(2 \mathrm{H}, \mathrm{m}), 7.62(1 \mathrm{H}, \mathrm{t}, J=7.2 \mathrm{~Hz}), 7.47(1 \mathrm{H}$, $\mathrm{d}, J=7.2 \mathrm{~Hz}), 7.34(1 \mathrm{H}, \mathrm{d}, J=7.8 \mathrm{~Hz}), 7.30(1 \mathrm{H}, \mathrm{s}), 7.19(1 \mathrm{H}, \mathrm{d}$, $J=8.4 \mathrm{~Hz}), 7.15(1 \mathrm{H}, \mathrm{d}, J=8.4 \mathrm{~Hz}), 7.03(1 \mathrm{H}, \mathrm{s}), 6.91(1 \mathrm{H}, \mathrm{d}, J=$ $8.4 \mathrm{~Hz}), 6.83(1 \mathrm{H}, \mathrm{d}, J=7.8 \mathrm{~Hz}), 5.81(1 \mathrm{H}, \mathrm{s}), 4.52(1 \mathrm{H}, \mathrm{d}, J=11.4$ $\mathrm{Hz}), 4.30$ (1H, d, $J=12.0 \mathrm{~Hz}), 4.23(1 \mathrm{H}, \mathrm{m}), 4.09(1 \mathrm{H}, \mathrm{m}), 3.88$ $(3 \mathrm{H}, \mathrm{s}), 3.74(2 \mathrm{H}, \mathrm{m}), 3.66(3 \mathrm{H}, \mathrm{s}), 2.62(2 \mathrm{H}, \mathrm{m}), 2.52(1 \mathrm{H}, \mathrm{m})$, $2.35(1 \mathrm{H}, \mathrm{m}), 1.84(2 \mathrm{H}, \mathrm{m}), 1.70(5 \mathrm{H}, \mathrm{m}), 1.58(1 \mathrm{H}, \mathrm{m}) .{ }^{13} \mathrm{C} \mathrm{NMR}$ $\left(P y-d_{5}\right): \delta 205.1(\mathrm{C}=\mathrm{O}), 191.4(\mathrm{C}=\mathrm{O}), 189.4(\mathrm{C}=\mathrm{O}), 179.9(\mathrm{C}=$ O), 148.8 (C), 148.6 (C), 147.1 (C), 147.0 (C), 142.9 (C), 142.2 (C), $138.2(\mathrm{CH}), 135.0(\mathrm{CH}), 132.1(\mathrm{C}), 131.8(\mathrm{CH}), 130.75(\mathrm{C}), 130.67$ (C), 130.45 (C), 128.9 (C), $128.2(\mathrm{CH}), 128.1(\mathrm{CH}), 125.3(\mathrm{CH})$, 123.6 (C), $122.9(\mathrm{CH}), 121.6(\mathrm{CH}), 120.4(\mathrm{CH}), 120.3(\mathrm{CH}), 116.7$ $(\mathrm{CH}), 116.4(\mathrm{CH}), 112.4(\mathrm{CH}), 111.5(\mathrm{CH}), 111.1(\mathrm{CH}), 100.7(\mathrm{CH})$, 84.2 (C), $77.5(\mathrm{C}), 74.1(\mathrm{C}), 73.5(\mathrm{CH}), 73.0(\mathrm{CH}), 64.5(\mathrm{CH}), 62.9$
(CH), 55.7 (OMe), 55.4 (OMe), $52.9(\mathrm{CH}), 51.4(\mathrm{CH}), 48.2\left(\mathrm{CH}_{2}\right)$, $47.7\left(\mathrm{CH}_{2}\right), 30.7\left(\mathrm{CH}_{2}\right), 30.4\left(\mathrm{CH}_{2}\right), 27.7\left(\mathrm{CH}_{2}\right), 27.0\left(\mathrm{CH}_{2}\right) . \mathrm{MS}$ [ESI-MS, positive mode]: found $m / z 930[\mathrm{M}+\mathrm{H}]^{+}$. HRMS [ESIMS, positive mode]: MF: $\mathrm{C}_{49} \mathrm{H}_{45} \mathrm{IN}_{3} \mathrm{O}_{8}$; found $\mathrm{m} / z 930.2252$ $[\mathrm{M}+\mathrm{H}]^{+}$[calcd. 930.2251].

\section{Spectral data of compound 7C $( \pm)$}

Obtained as white solid; yield: $50 \%(607 \mathrm{mg}) ; \mathrm{mp}: 204-206^{\circ} \mathrm{C} ; R_{\mathrm{f}}$ 0.49 (5\% MeOH in $\left.\mathrm{CHCl}_{3}\right) ;{ }^{1} \mathrm{H}$ NMR $\left(P y-d_{5}\right): \delta 11.78(1 \mathrm{H}, \mathrm{s},-\mathrm{NH})$, $8.08(1 \mathrm{H}, \mathrm{dd}, J=2.4,6.6 \mathrm{~Hz}), 8.05(1 \mathrm{H}, \mathrm{dd}, J=2.4,8.4 \mathrm{~Hz}), 7.83$ $(1 \mathrm{H}, \mathrm{dd}, J=2.4,8.4 \mathrm{~Hz}), 7.74(1 \mathrm{H}, \mathrm{m}), 7.62(2 \mathrm{H}, \mathrm{m}), 7.47(1 \mathrm{H}, \mathrm{dd}$, $J=1.8,7.8 \mathrm{~Hz}), 7.41(1 \mathrm{H}, \mathrm{dd}, J=1.8,7.8 \mathrm{~Hz}), 7.31(1 \mathrm{H}, \mathrm{s}), 7.26$ $(1 \mathrm{H}, \mathrm{d}, J=8.4 \mathrm{~Hz}), 7.18(2 \mathrm{H}, \mathrm{m}), 7.12(1 \mathrm{H}, \mathrm{d}, J=7.8 \mathrm{~Hz}), 6.96$ $(1 \mathrm{H}, \mathrm{d}, J=7.8 \mathrm{~Hz}), 6.91(2 \mathrm{H}, \mathrm{m}), 5.86(1 \mathrm{H}, \mathrm{s}), 4.46(1 \mathrm{H}, \mathrm{m}), 4.32$ $(2 \mathrm{H}, \mathrm{m}), 4.11(1 \mathrm{H}, \mathrm{m}), 3.87(3 \mathrm{H}, \mathrm{s}), 3.82(3 \mathrm{H}, \mathrm{s}), 3.76(2 \mathrm{H}, \mathrm{m})$, $3.49(1 \mathrm{H}, \mathrm{m}), 3.24(1 \mathrm{H}, \mathrm{d}, J=10.8 \mathrm{~Hz}), 3.20(1 \mathrm{H}, \mathrm{d}, J=11.4 \mathrm{~Hz})$, $3.02(1 \mathrm{H}, \mathrm{m}), 2.57(2 \mathrm{H}, \mathrm{m}), 1.86(1 \mathrm{H}, \mathrm{m}), 1.71(2 \mathrm{H}, \mathrm{m}), 1.64(1 \mathrm{H}$, m). ${ }^{13} \mathrm{C}$ NMR $\left(P y-d_{5}\right): \delta 205.0(\mathrm{C}=\mathrm{O}), 190.9(\mathrm{C}=\mathrm{O}), 190.5(\mathrm{C}=\mathrm{O})$, 180.9 (C=O), 149.8 (C), 149.6 (C), 148.3 (C), 147.9 (C), 143.9 (C), 143.4 (C), 133.2 (C), $132.51(\mathrm{CH}), 132.47$ (C), 131.2 (C), 131.0 (C), $130.3(\mathrm{C}), 129.7(\mathrm{CH}), 128.7(\mathrm{CH}), 128.6(\mathrm{CH}), 126.8(\mathrm{CH})$, $126.4(\mathrm{C}), 126.2(\mathrm{CH}), 125.1(\mathrm{CH}), 122.4(\mathrm{CH}), 121.9(\mathrm{CH}), 121.7$ $(\mathrm{CH}), 121.5(\mathrm{CH}), 117.5(\mathrm{CH}), 117.3(\mathrm{CH}), 111.9(\mathrm{CH}), 111.3(\mathrm{CH})$, $110.6(\mathrm{CH}), 100.9(\mathrm{CH}), 78.7(\mathrm{C}), 77.4(\mathrm{CH}), 74.4(\mathrm{C}), 73.8(\mathrm{CH})$, $63.8(\mathrm{CH}), 61.2(\mathrm{CH}), 56.3(\mathrm{OMe}), 56.2(\mathrm{OMe}), 55.9\left(\mathrm{CH}_{2}\right), 53.0$ $(\mathrm{CH}), 52.4(\mathrm{CH}), 48.4\left(\mathrm{CH}_{2}\right), 37.2\left(\mathrm{CH}_{2}\right), 31.3\left(\mathrm{CH}_{2}\right), 27.9\left(\mathrm{CH}_{2}\right)$. MS [ESI-MS, positive mode]: found $m / z 822[\mathrm{M}+\mathrm{H}]^{+}, 844[\mathrm{M}+$ $\mathrm{Na}]^{+}$. HRMS [ESI-MS, positive mode]: $\mathrm{MF}: \mathrm{C}_{48} \mathrm{H}_{43} \mathrm{~N}_{3} \mathrm{O}_{8} \mathrm{~S}$; found $m / z$ 844.2670 [M + Na] $]^{+}$[calcd. 844.2669].

\section{Spectral data of compound 7D $( \pm)$}

Obtained as yellowish white solid; yield: 49\% (588 mg); mp: 160-162 ${ }^{\circ} \mathrm{C} ; R_{\mathrm{f}} 0.48$ (5\% $\mathrm{MeOH}$ in $\left.\mathrm{CHCl}_{3}\right)$; UV absorption maximas $\left(\lambda_{\max } \mathrm{nm}\right.$, methanol) $284,254,215 ;{ }^{1} \mathrm{H}$ NMR $\left(P y-d_{5}\right)$ : $\delta 8.05(2 \mathrm{H}, \mathrm{m}), 7.87(1 \mathrm{H}, \mathrm{d}, J=8.4 \mathrm{~Hz}), 7.76(1 \mathrm{H}, \mathrm{d}, J=8.4 \mathrm{~Hz})$, $7.65(1 \mathrm{H}, \mathrm{t}, J=7.8 \mathrm{~Hz}), 7.60(1 \mathrm{H}, \mathrm{d}, J=7.2 \mathrm{~Hz}), 7.39(1 \mathrm{H}, \mathrm{d}, J=$ $7.8 \mathrm{~Hz}), 7.31(1 \mathrm{H}, \mathrm{d}, J=1.2 \mathrm{~Hz}), 7.27(1 \mathrm{H}, \mathrm{d}, J=8.4 \mathrm{~Hz}), 7.20$ $(1 \mathrm{H}, \mathrm{m}), 7.15(1 \mathrm{H}, \mathrm{s}), 7.07(2 \mathrm{H}, \mathrm{m}), 7.01(1 \mathrm{H}, \mathrm{d}, J=7.8 \mathrm{~Hz}), 6.96$ $(1 \mathrm{H}, \mathrm{dd}, J=1.2,7.8 \mathrm{~Hz}), 5.88(1 \mathrm{H}, \mathrm{s}), 4.46(1 \mathrm{H}, \mathrm{d}, J=12.6 \mathrm{~Hz})$, $4.33(2 \mathrm{H}, \mathrm{m}), 4.12(1 \mathrm{H}, \mathrm{m}), 3.88(3 \mathrm{H}, \mathrm{s}), 3.76(2 \mathrm{H}, \mathrm{m}), 3.73(3 \mathrm{H}$, $\mathrm{s}), 3.70(1 \mathrm{H}, \mathrm{m}), 3.24(1 \mathrm{H}, \mathrm{d}, J=10.8 \mathrm{~Hz}), 3.12(1 \mathrm{H}, \mathrm{d}, J=11.4$ $\mathrm{Hz}), 3.00(1 \mathrm{H}, \mathrm{m}), 2.72(1 \mathrm{H}, \mathrm{m}), 2.63(1 \mathrm{H}, \mathrm{m}), 2.13(3 \mathrm{H}, \mathrm{s}), 1.87$ $(1 \mathrm{H}, \mathrm{m}), 1.77(2 \mathrm{H}, \mathrm{m}), 1.68(1 \mathrm{H}, \mathrm{m}) .{ }^{13} \mathrm{C} \mathrm{NMR}\left(P y-d_{5}\right): \delta 204.5$ $(\mathrm{C}=\mathrm{O}), 192.0(\mathrm{C}=\mathrm{O}), 188.1(\mathrm{C}=\mathrm{O}), 180.4(\mathrm{C}=\mathrm{O}), 149.0(\mathrm{C})$, 148.7 (C), 147.6 (C), 147.1 (C), 142.8 (C), 140.8 (C), 132.8 (C), 131.9 (CH), 131.8 (C), 130.8 (C), 130.5 (C), 130.3 (C), 129.8 (CH, C), $128.1(\mathrm{CH}), 128.0(\mathrm{CH}), 127.3(\mathrm{CH}), 126.1(\mathrm{C}), 125.5$ $(\mathrm{CH}), 124.3(\mathrm{CH}), 121.8(\mathrm{CH}), 120.9(\mathrm{CH}), 120.6(\mathrm{CH}), 116.8(\mathrm{CH})$, $116.5(\mathrm{CH}), 111.4(\mathrm{CH}), 110.8(\mathrm{CH}), 109.9(\mathrm{CH}), 100.4(\mathrm{CH}), 78.2$ (C), $77.1(\mathrm{CH}), 74.1(\mathrm{C}), 73.1(\mathrm{CH}), 64.1(\mathrm{CH}), 60.5(\mathrm{CH}), 55.6$ (OMe), $55.5(\mathrm{OMe}), 55.2\left(\mathrm{CH}_{2}\right), 51.8(\mathrm{CH}), 51.6(\mathrm{CH}), 47.7\left(\mathrm{CH}_{2}\right)$, $36.8\left(\mathrm{CH}_{2}\right), 30.8\left(\mathrm{CH}_{2}\right), 27.7\left(\mathrm{CH}_{2}\right), 20.8\left(\mathrm{CH}_{3}\right) . \mathrm{MS}$ [ESI-MS, positive mode]: found $\mathrm{m} / \mathrm{z} 836[\mathrm{M}+\mathrm{H}]^{+}$. HRMS [ESI-MS, positive mode]: MF: $\mathrm{C}_{49} \mathrm{H}_{46} \mathrm{~N}_{3} \mathrm{O}_{8} \mathrm{~S}$; found $\mathrm{m} / z$ 836.3009 $[\mathrm{M}+\mathrm{H}]^{+}$ [calcd. 836.3006]. 


\section{Conflicts of interest}

There are no conflicts of interest to declare.

\section{Acknowledgements}

A. H. and B. Y. are recipients of Research Fellowships in the form of Young Scientist Grant (YSS/2015/001141) and NPDF/ 2016/000088 from SERB-DST, India. Our thanks are due to Director NIPER and IICB for giving laboratory and instrumental facility, and to Dr. B. Achari (Ex-Scientist, CSIR) for helpful suggestions.

\section{Notes and references}

1 (a) S. Fergus, A. Bender and D. R. Spring, Curr. Opin. Chem. Biol., 2005, 9, 304-309; (b) D. R. Spring, Chem. Soc. Rev., 2005, 34, 472-482; (c) S. L. Schreiber, Bioorg. Med. Chem., 1998, 6, 1127-1152; (d) S. L. Schreiber, Chem. Eng. News, 2003, 81, 51-61; (e) D. P. Walsh and Y. T. Chang, Chem. Rev., 2006, 106, 2476; (f) D. S. Tan, Nat. Chem. Biol., 2005, 1, 74-84.

2 M. Feher and J. M. Schmidt, J. Chem. Inf. Comput. Sci., 2003, 43, 218-227.

3 S. J. Haggarty, Curr. Opin. Chem. Biol., 2005, 9, 296-303.

4 (a) S. L. Schreiber, Nature, 2009, 457, 153-154; (b) G. T. Le, G. Abbenante and B. Becker, Drug Discovery Today, 2003, 8, 701-709; (c) M. Lajiness and I. Watson, Curr. Opin. Chem. Biol., 2008, 12, 366-371.

5 (a) R. J. Spandl, A. Bender and D. R. Spring, Org. Biomol. Chem., 2008, 6, 1149-1158; (b) Diversity-Oriented Synthesis in Solid-Phase Organic Synthesis: Concepts, Strategies, and Applications, ed. P. H. Toy and Y. Lam, John Wiley \& Sons, Inc, 1st edn, 2012; (c) V. Abet, A. Mariani, F. R. Truscott, S. Britton and R. Rodriguez, Bioorg. Med. Chem., 2014, 22, 4474-4489; (d) M. D. Burke and S. L. Schreiber, Angew. Chem., Int. Ed., 2004, 43, 46-58.

6 (a) G. Rainoldi, F. Begnini, M. D. Munnik, L. L Presti, C. M. L. V. Velde, R. Orru, G. Lesma, E. Ruijter and A. Silvani, ACS Comb. Sci., 2018, 20, 98-105; (b) S. Kanchithalaivan, R. V. Sumesh and R. R. Kumar, ACS Comb. Sci., 2014, 16, 566-572.

7 (a) H. Liu, S. Ueta, F. Yagishita, M. Nishiuchi and Y. Kawamura, Tetrahedron, 2015, 71, 3614-3618; (b)
S. Lanka, S. Thennarasu and P. T. Perumal, Tetrahedron Lett., 2014, 55, 2585-2588; (c) R. Rajesh and R. Raghunathan, Tetrahedron Lett., 2010, 51, 5845-5848; (d) Q. Lu, X. Huang, G. Song, C. M. Sun, J. P. Jasinski, A. C. Keeley and W. Zhang, ACS Comb. Sci., 2013, 15, 350355; (e) A. Srivastav, L. Aggarwal and N. Jain, ACS Comb. Sci., 2015, 17, 39-48.

8 A. Hazra, S. Mondal, A. Maity, S. Naskar, P. Saha, R. Paira, K. B. Sahu, P. Paira, S. Ghosh, C. Sinha, A. Samanta, S. Banerjee and N. B. Mondal, Eur. J. Med. Chem., 2011, 46, 2132-2140.

9 A. Hazra, Y. P. Bharitkar, A. Maity, S. Mondal and N. B. Mondal, Tetrahedron Lett., 2013, 54, 4339-4342.

10 (a) A. Hazra, P. Paira, K. B. Sahu, S. Naskar, P. Saha, R. Paira, S. Mondal, A. Maity, P. Luger, M. Weber, N. B. Mondal and S. Banerjee, Tetrahedron Lett., 2010, 51, 1585-1588; (b) A. Hazra, Y. P. Bharitkar, D. Chakraborty, S. K. Mondal, N. Singal, S. Mondal., A. Maity, R. Paira, S. Banerjee and N. B. Mondal, ACS Comb. Sci., 2013, 15, 41-48.

11 Y. P. Bharitkar, S. Kanhar, N. Suneel, S. K. Mondal, A. Hazra and N. B. Mondal, Mol. Diversity, 2015, 19, 251-261.

12 Y. P. Bharitkar, M. Das, N. Kumari, M. P. Kumari, A. Hazra, S. Bhayye, R. Natarajan, S. Shah, S. Chatterjee and N. B. Mondal, Org. Lett., 2015, 17, 4440-4443.

13 S. K. Dey, D. Bose, A. Hazra, S. Naskar, A. Nandy, R. N. Munda, S. Das, N. Chatterjee, N. B. Mondal, S. Banerjee and K. D. Saha, PLoS One, 2013, 8, e58055.

14 D. Chakraborty, A. Maity, C. K. Jain, A. Hazra, Y. P. Bharitkar, T. Jha, H. K. Majumder, S. Roychoudhury and N. B. Mondal, Med. Chem. Commun., 2015, 6, 702-707.

15 M. H. M. Helal, N. S. Ahmed, M. S. Elwessaly and Y. A. Ammar, Arch. Pharm. Chem. Life Sci., 2014, 347, 123133.

16 S. N. A. Bukhari, I. B. Jantan, M. Jasamai, W. Ahmad and M. W. B. Amjad, Int. J. Med. Sci., 2013, 13, 501-513.

17 D. K. Agrawal and P. K. Mishra, Med. Res. Rev., 2010, 30, 818860.

18 S. Mishra, K. Karmodiya, N. Surolia and A. Surolia, Bioorg. Med. Chem., 2008, 16, 2894-2902.

19 Y. P. Bharitkar, S. Datta, S. Sett, N. Marathee, P. Khan, A. Hazra, M. Singh, A. Sahoo, S. Ghosh, S. Mondal, A. K. Mitra, V. Ravichandiran and N. B. Mondal, Chem. Biol. Interface, 2017, 7, 19-31. 